\title{
Triangulated surfaces in triangulated categories
}

\author{
T. Dyckerhoff*; M. Kapranov*
}

October 15, 2013

\begin{abstract}
For a triangulated category $\mathcal{A}$ with a 2-periodic dg-enhancement and a triangulated oriented marked surface $S$, we introduce a dg-category $\mathscr{F}(S, \mathcal{A})$ parametrizing systems of exact triangles in $\mathcal{A}$ labelled by triangles of $S$. Our main result is that $\mathscr{F}(S, \mathcal{A})$ is independent of the choice of a triangulation of $S$ up to essentially unique Morita equivalence. In particular, it admits a canonical action of the mapping class group. The proof is based on general properties of cyclic 2-Segal spaces.

In the simplest case, where $\mathcal{A}$ is the category of 2 -periodic complexes of vector spaces, $\mathscr{F}(S, \mathcal{A})$ turns out to be a purely topological model for the Fukaya category of the surface $S$. Therefore, our construction can be seen as implementing a 2dimensional instance of Kontsevich's program on localizing the Fukaya category along a singular Lagrangian spine.
\end{abstract}

\section{Contents}

\begin{tabular}{l|l} 
Introduction & 2
\end{tabular}

1 Background on the homotopy theory of dg-structures 9

1.1 Model structures on the category of differential $\mathbb{Z}$-graded categories. . . . 9

1.2 The 2 -periodic case . . . . . . . . . . . . . . . . . . 14

2 Loop factorizations 15

$2.1 \mathbb{Z}_{+}$-categories and the cyclic category . . . . . . . . . . 15

2.2 Loop factorizations in $\mathbb{Z}_{+}$-categories . . . . . . . . . . . . . . 17

2.3 The cyclic model for $D^{(2)}\left(A_{n}\right.$-mod) via matrix factorizations . . . . . . 18

2.4 The dg-categories $\mathcal{E}^{n}$ and their cocyclic structure . . . . . . . . . . . . 21

2.5 Cyclic duality and Morita duality . . . . . . . . . . . 27

*Department of Mathematics, Yale University, 10 Hillhouse Avenue, New Haven CT 06520 USA, email: tobias.dyckerhoff@yale.edu, mikhail.kapranov@yale.edu 
3 Cyclic 2-Segal objects 2

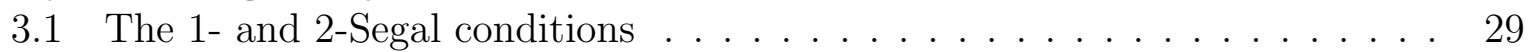

3.2 Examples of cyclic 2-Segal objects . . . . . . . . . . . . . . . . 31

3.3 Background on triangulated surfaces and ribbon graphs . . . . . . . . . . 33

3.4 Cyclic membrane spaces . . . . . . . . . . . . . . . . 37

4 Application: Fukaya categories 442

4.1 Topological Fukaya categories . . . . . . . . . . . . . . . . . . . . . . . . . . . . . .

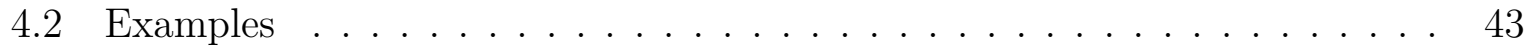

5 Application: Waldhausen S-construction 49 


\section{Introduction}

The goal of this paper is to study a certain "2-dimensional symmetry" built into the very foundations of triangulated categories and thus of homological algebra more generally. To make it manifest, we represent exact triangles in a triangulated category $\mathcal{D}$, in the dual fashion:

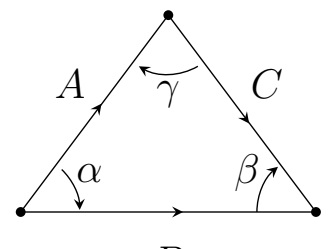

$$
\Longleftrightarrow \quad A \stackrel{\alpha}{\longrightarrow} B \stackrel{\beta}{\longrightarrow} C \stackrel{\gamma}{\longrightarrow} A[1]
$$

$B$

That is, we assign objects to oriented edges of geometric triangles, and morphisms to their angles. A morphism of degree 1 is represented by an angle formed by two edges with different directions (one incoming, one outgoing). The advantage of this dual point of view is that the most fundamental types of diagrams are now represented by collections of geometric triangles of the most basic shapes.

Example 0.2. The two halves of an octahedron are represented by two triangulations of a 4-gon. The octahedral axiom is now interpreted as switching from one triangulation to the other (flip):
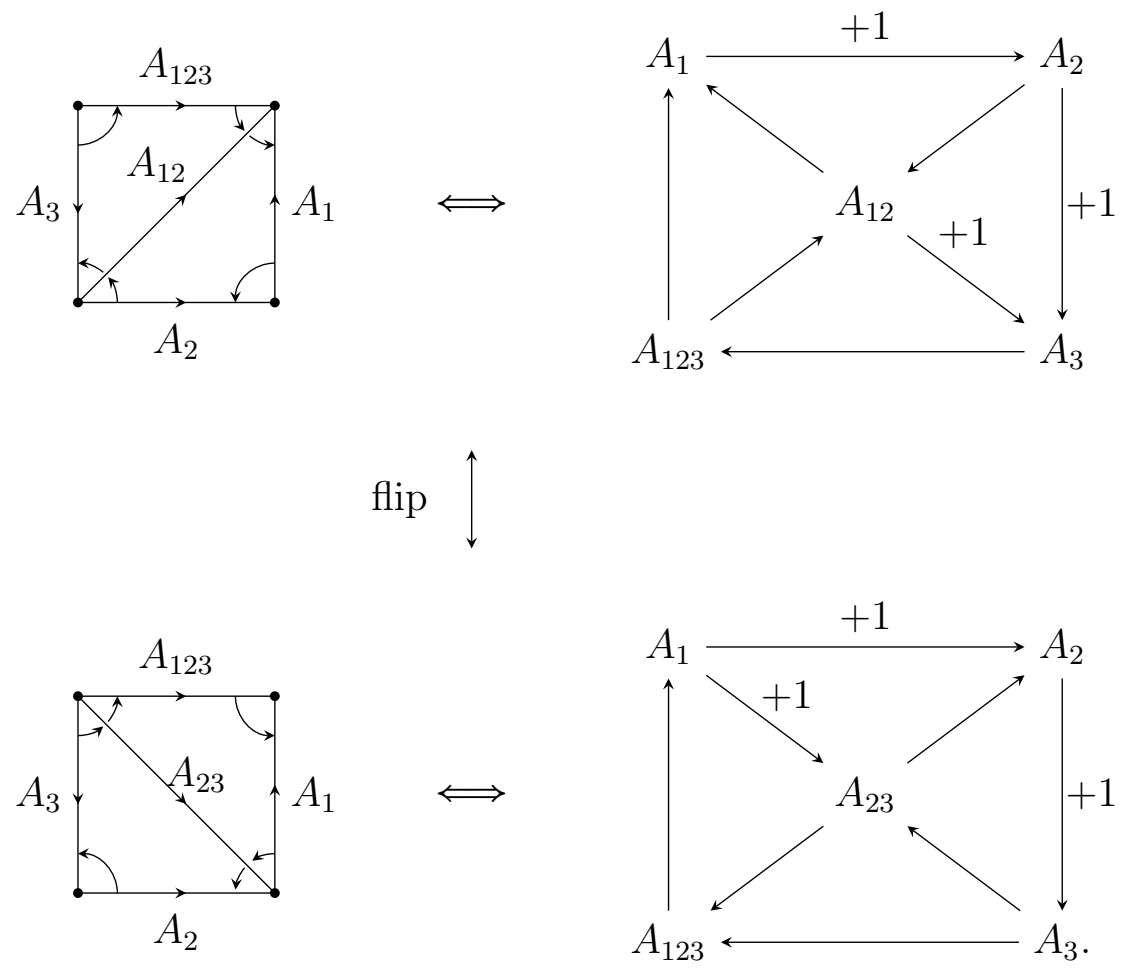

Example 0.3. A Postnikov system in $\mathcal{D}$ is a diagram of exact triangles representing an object $A_{12 \ldots n}$ as an iterated extension of ("tower of fibrations" with fibers being) the 
given objects $A_{1}, \ldots, A_{n}$, see [GM03], Ch. 4, §2. Note that there are several possible types of Postnikov systems, see loc. cit. In our approach, these correspond to different triangulations of the $(n+1)$-gon. The octahedral axiom thus allows us to pass from any one type to any other by a sequence of flips on 4-gons.
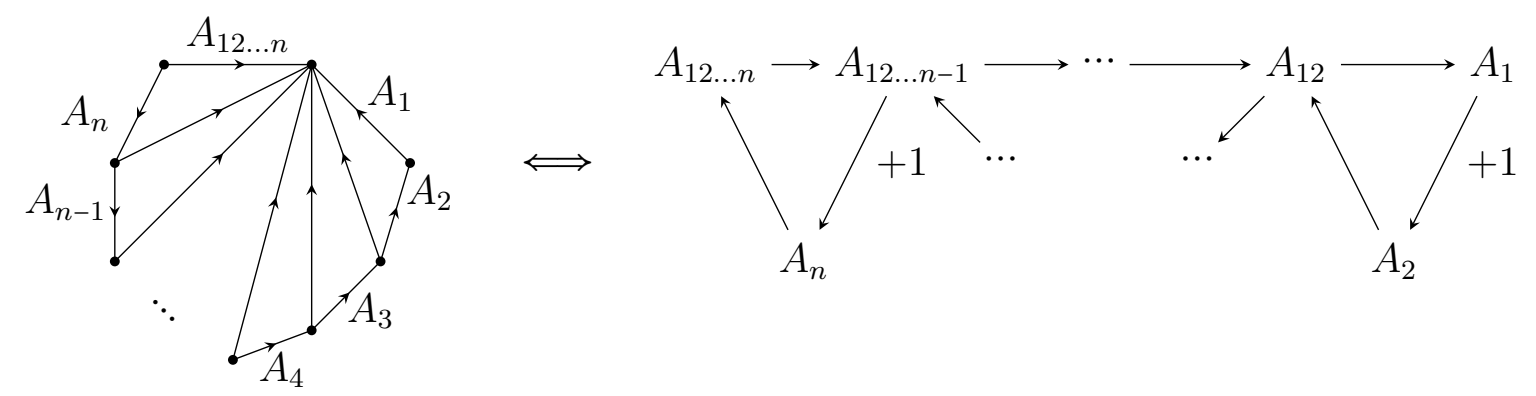

This 2-dimensional symmetry becomes even more pronounced, if $\mathcal{D}$ is 2-periodic, i.e., the shift functor $\Sigma: A \mapsto A[1]$ squares to the identity. In this case we can freely switch the directions of edges in the geometric triangle representing an exact one as above, by postulating that such switches amount to applying $\Sigma$ :

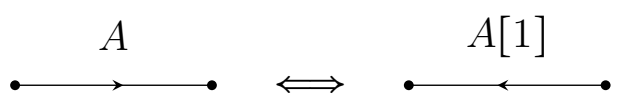

The really important remaining datum is purely 2-dimensional: it is the orientation of the geometric triangle itself, which determines the directions of the morphisms between the objects on its edges. We can therefore consider surface Postnikov systems: diagrams of exact triangles in $\mathcal{D}$, whose associated geometric triangles form a curvilinear triangulation $\mathcal{T}$ of an oriented topological surface $S$, possibly with boundary.

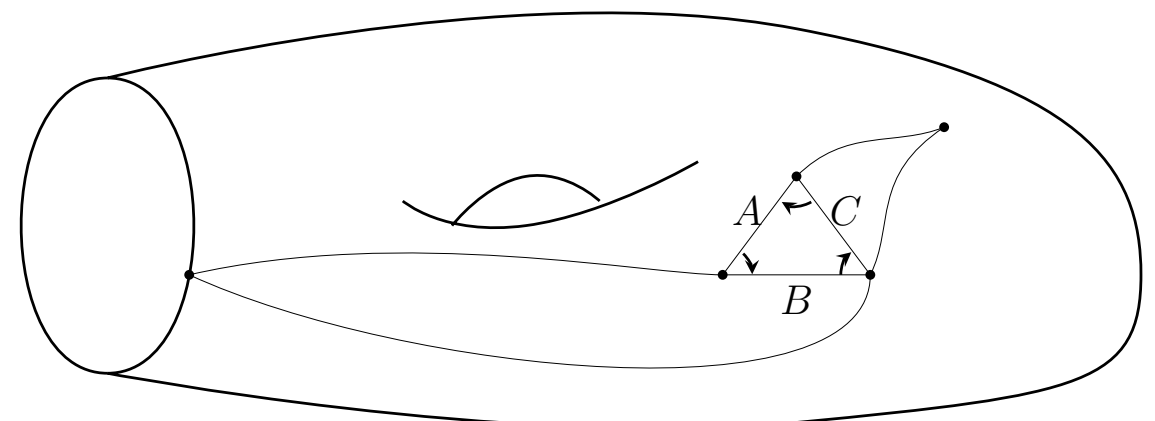

Ordinary Postnikov systems are obtained when $S$ is a disk and all the vertices of $\mathcal{T}$ are on $\partial S$. Standard results of Teichmüller theory imply that any two triangulations of $S$ with the same underlying set of vertices $M$ are connected by a sequence of flips. This suggests that an appropriately defined classifying space of surface Postnikov systems depends, in a very canonical way, only on the oriented surface $(S, M)$ and not on a chosen 
triangulation $\mathcal{T}$, in particular, that it is acted upon by the mapping class group of $(S, M)$. In the present paper we make this statement precise and provide a proof. The resulting theory turns out to be related to subjects such as Fukaya categories, matrix factorizations and mirror symmetry.

In order to have good classifying spaces of exact diagrams in $\mathcal{D}$, it seems unavoidable to assume that $\mathcal{D}$ comes with an enhancement, a certain refinement of the graded abelian groups $\operatorname{Hom}_{\mathcal{D}}(A, \Sigma \bullet B)$. In this paper we mostly work with dg-enhancements (\$1.1) which allows us to use techniques from the Morita homotopy theory of dg-categories Tab07, Toë07] such as model structures, simplicial mapping spaces, homotopy limits, etc.

In this setting, given any triangulation $\mathcal{T}$ of $(S, M)$, we can form the universal Postnikov system of type $\mathcal{T}$ which is a 2-periodic dg-category $L \mathcal{E}^{\mathcal{T}}$ with the following "universal property": Given any perfect 2-periodic dg category $\mathcal{A}$, enhancing a triangulated category $\mathcal{D}$, the classifying space of surface Postnikov systems of type $\mathcal{T}$ with values in $\mathcal{A}$ is given as the simplicial mapping space

$$
\operatorname{Post}^{\mathcal{T}}(\mathcal{A}):=\operatorname{Map}\left(L \mathcal{E}^{\mathcal{T}}, \mathcal{A}\right)
$$

in the category of 2-periodic dg categories, localized along Morita equivalences.

Our main result says that, up to Morita equivalence, $L \mathcal{E}^{\mathcal{T}}$ does not depend on $\mathcal{T}$, so that we obtain an object

$$
\mathscr{F}^{(S, M)} \simeq L \mathcal{E}^{\mathcal{T}} \in \mathrm{Hmo}^{(2)} .
$$

which, up to unique isomorphism, only depends on $(S, M)$. Here, $\mathrm{Hmo}^{(2)}$ is the Morita homotopy category of 2-periodic dg-categories. In particular, the mapping class group of $(S, M)$ acts on $\mathscr{F}(S, M)$ by automorphisms in $\mathrm{Hmo}^{(2)}$.

We can refine construction (0.4) to form the classifying dg-category of Postnikov systems of type $\mathcal{T}$ in $\mathcal{A}$

$$
\underline{\operatorname{Post}}^{\mathcal{T}}(\mathcal{A}):=R \underline{\operatorname{Hom}}\left(L \mathcal{E}^{\mathcal{T}}, \mathcal{A}\right)
$$

where $R \underline{\text { Hom }}$ denotes Toën's internal Hom for the category of dg-categories. For the same reasons as above, this 2-periodic dg-category is acted upon by the mapping class group of the surface $(S, M)$. In fact, in both cases, the action of the mapping class group is coherent in the sense of homotopy theory.

As pointed out to us by M. Kontsevich, the dg-category $\mathscr{F}(S, M)$ is nothing but a version of the Fukaya category of the surface $S-\left(M \cap S^{\circ}\right)$ obtained by removing the points of $M$ lying in the interior of $S$. The representation of $\mathscr{F}(S, M)$ as $L \mathcal{E}^{\mathcal{T}}$ provides a rigorous implementation of an instance of his program of "localizing the Fukaya category along a singular Lagrangian spine" [Kon09, Kon09']. More generally, he considered a $2 d-$ dimensional symplectic manifold $(U, \omega)$ which can be contracted onto a possibly singular Lagrangian subvariety $L \subset U$ by the flow along a vector field $\xi$ satisfying $\operatorname{Lie}_{\xi}(\omega)=-\omega$. In such a situation he suggested to construct a "cosheaf of dg-categories" $\Phi_{L}$ on $L$, refining the Fukaya category $\mathcal{F}(U)$, which should be recovered as the category of global sections $\Phi_{L}(L)$. In particular, different choices of $L$ should lead to different realizations of $\mathcal{F}(U)$. 
Our situation corresponds to the simplest case $d=1$ when $U=S-M$, where $(S, M)$ is a marked surface with $\partial S=\varnothing$, which we consider as a symplectic manifold with respect to some 2 -form $\omega$. A triangulation $\mathcal{T}$ of $(S, M)$ gives then a 3 -valent dual graph $L \subset U$ defined up to isotopy and Lagrangian because $\operatorname{dim}(L)=1$.

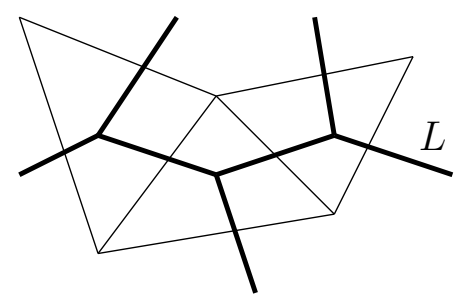

Our $L \mathcal{E}^{\mathcal{T}}$ corresponds to $\Phi_{L}(L)$. Further, the "local" nature of $\Phi_{L}$ in Kontsevich's proposal corresponds to our construction of $L \mathcal{E}^{\mathcal{T}}$ by gluing it out of local data, a certain system of 2-periodic dg-categories $\mathcal{E}^{\bullet}=\left(\mathcal{E}^{n}\right)_{n \geq 0}$ such that:

(1) $\mathcal{E}^{\bullet}$ is a cocyclic object, in the sense of A. Connes [Con94], in the category dgcat ${ }^{(2)}$ of 2-periodic dg-categories. In particular, the group $\mathbb{Z} /(n+1)$ acts on $\mathcal{E}^{n}$ by automorphisms of dg-categories.

(2) For every $n \geq 0$, the dg-category $\mathcal{E}^{n}$ is Morita equivalent to a dg-enhancement of $D^{(2)}\left(A_{n}\right.$-mod), the 2-periodic derived category of representations of the quiver $A_{n}$. The action of the generator of $\mathbb{Z} /(n+1)$ corresponds to the Coxeter functor.

(3) The cosimplicial object underlying $\mathcal{E} \bullet$ is 2-coSegal in the sense of [HSS1].

Property (1) is responsible for the fact that the construction $L \mathcal{E}^{\mathcal{T}}$ does only depend on the orientation of each triangle of $\mathcal{T}$ induced from the orientation of the surface $S$ and not on any particular orientations of its edges. Condition (2) corresponds to the requirement in [Kon09] that the stalk of $\Phi_{L}$ at a ramification point of $L$ with valency $n+1$ should be a version of $D\left(A_{n}\right.$-mod). The 2-coSegal property (3) ensures the coherent independence of $L \mathcal{E}^{\mathcal{T}}$ on $\mathcal{T}$ (or, equivalently, of $\Phi_{L}$ on $L$ ).

We call the dg-category $\mathscr{F}(S, M)$ the topological coFukaya category of the marked oriented surface $(S, M)$. Dually, for any perfect 2-periodic dg-category $\mathcal{A}$, the dg-category

$$
R \underline{\operatorname{Hom}}\left(\mathscr{F}^{(S, M)}, \mathcal{A}\right)
$$

is called the topological Fukaya category of $(S, M)$ with coefficients in $\mathcal{A}$. In the case where $\mathcal{A}$ is the dg category $\operatorname{Perf}_{\mathbf{k}}^{(2)}$ of 2 -periodic perfect complexes of $\mathbf{k}$-vector spaces, we introduce the notation

$$
\mathscr{F}_{(S, M)}=R \underline{\operatorname{Hom}}\left(\mathscr{F}^{(S, M)}, \operatorname{Perf}_{\mathbf{k}}^{(2)}\right)
$$

and refer to this category as the topological Fukaya category of $(S, M)$. In other words, the dg-category $\mathscr{F}_{(S, M)}$ is the Morita dual of $\mathscr{F}(S, M)$. The terminology is chosen to reflect the descent properties of these constructions: The choice of a spanning Ribbon graph $\Gamma$ of 
the surface $(S, M)$ can be regarded as a combinatorial way of encoding an open covering of the surface. The Morita equivalences

$$
\begin{aligned}
& \mathscr{F}^{(S, M)} \simeq L \mathcal{E}^{\Gamma} \simeq \operatorname{holim}_{\left\{\Lambda^{n} \rightarrow \Lambda^{\Gamma}\right\}}^{\text {dgcat }^{(2)}} \mathcal{E}^{n} \\
& \mathscr{F}_{(S, M)} \simeq R \mathcal{E}_{\Gamma} \simeq \operatorname{holim}_{\left\{\Lambda^{n} \rightarrow \Lambda^{\Gamma}\right\}}^{\text {dgcat }^{(2)}} \mathcal{E}_{n}
\end{aligned}
$$

are immediate by our construction of the topological Fukaya category as a homotopy Kan extension, and assign a precise meaning to the statement that the topological (co)Fukaya category is a homotopy (co)sheaf with values in dg-categories. The homotopy limits in (0.6) and (0.7) are taken with respect to the Morita model structure and can be effectively computed using standard techniques from the theory of model categories. We illustrate this in 4.2 where we investigate some examples appearing on Kontsevich's list [Kon09', Pictures].

For our constructions to work, it is crucial that the system $\mathcal{E} \bullet$ of dg-categories satisfies conditions (1),(2), and (3) above. Note that the most immediate dg-enhancements of $D^{(2)}\left(A_{n}\right.$-mod $)$ do not have manifest cyclic symmetry. From the symplectic point of view, it is known that $D^{(2)}\left(A_{n}\right.$-mod) is the "Fukaya-Seidel category of the unit disk $|z| \leq 1$ equipped with the potential $z^{n+1} "$ (the 1-dimensional $A_{n}$-singularity). However, one does not obtain a suitable definition of $\mathcal{E}^{n}$ on this path either. Indeed, the definition of Seidel Sei08] requires choosing, first, a deformation of the singularity, i.e., a generic polynomial $f(z)=z^{n+1}+\sum_{i=0}^{n} a_{i} z^{i}$ and, second, an ordered basis of (0-dimensional) vanishing cycles of $f$, which, again, breaks the cyclic symmetry.

Instead, we define $\mathcal{E}^{n}$ in terms of matrix factorizations, in the sense of D. Eisenbud, of $z^{n+1}$, slightly modifying the setup of [Tak05]. Our motivation for this approach is that this matrix factorization category can be interpreted as the homological mirror of the above mentioned Fukaya-Seidel category, thus mirror symmetry between LandauGinzburg models and matrix factorizations is locally built into our constructions from the very outset. Our construction uses a new concept of loop factorization in V. Drinfeld's $\mathbb{Z}_{+}$-categories and is explained in detail in $\$ 2$.

The concept of a 2-Segal object was introduced in [HSS1 as a unifying concept for various situations when some object is defined in terms of a choice of a triangulation but ends up not depending on this choice in a coherent way. In the case of 2-Segal simplicial objects, treated in loc. cit., we deal with triangulations of plane polygons and related instances of associativity, such as, e.g., in the context of Hall algebras. The example that motivated our study of 2-Segal spaces in [HSS1] was the Waldhausen Sconstruction, a simplicial space which plays a fundamental role in algebraic K-theory, see Gil81]. In [HSS1] we introduced a generalization of the S-construction encompassing arbitrary stable $\infty$-categories [Lur06, Lur11]. The present work grew out of our heuristic observation that for 2-periodic perfect dg categories, the S-construction has a cyclic, and not just a simplicial structure. Passing from simplicial to cyclic objects allows one to extend the polygon triangulations to triangulations of arbitrary marked oriented surfaces in a non-ambiguous way which, applied to the S-construction, leads to a precise variant of 
the surface Postnikov systems described above. The relevant constructions for the present work are provided in \$3. A more detailed account of the general theory will be given in HSS2].

The starting point of this project was a suggestion of J. Lurie to rigorously establish the additional cyclic symmetry of the S-construction by constructing a cocyclic dg-category which corepresents it in $\operatorname{dgcat}^{(2)}$. The object $\mathcal{E} \bullet$ provides a solution, in the sense that, given a 2-periodic perfect dg-category $\mathcal{A}$, the simplicial space underlying the cyclic space $\operatorname{Map}(\mathcal{E} \bullet \mathcal{A})$ is weakly equivalent to the Waldhausen $\mathrm{S}$-construction of $\mathcal{A}$. This relies on a comparison result between pre-triangulated dg-categories and stable $\infty$-categories which has been carried out by G. Faonte [Fao13. A more detailed analysis will be given in [HSS2].

In conclusion, we find it remarkable that the observation

$$
\left(\begin{array}{c}
\text { Axioms of homological } \\
\text { algebra }
\end{array}\right) \longleftrightarrow\left(\begin{array}{c}
\text { Flips of } \\
2 d \text { triangulations }
\end{array}\right)
$$

naturally leads to a topological variant of the Fukaya category. This phenomenon seems to be potentially appealing even to someone with no symplectic motivation whatsoever.

We would like to point out that there have been various projects addressing Kontsevich's localization program for 2-dimensional symplectic manifolds. We refer the reader to [STZ11] and the references therein. In higher dimensions, the general problem of localization on a given spine is treated in Nad11. Very recently, a construction similar to ours has been given in the context of $A_{\infty}$-categories Nad13. The main novelty in our approach is the 2-Segal property which reflects, in a conceptually clear way, the fact that the category we construct is a topological invariant of the marked surface, coherently independent of a chosen spine. Finally, we wish to mention that, as we were informed by J. Lurie, he himself has, in joint work with A. Preygel, found a coparacyclic version of the cocyclic object $\mathcal{E} \bullet$ which is suitable for an analysis from the point of view of $\infty$-categories and relates to classical concepts from homotopy theory such as the J-homomorphism.

Acknowledgements. We are very grateful to J. Lurie for his interest and for many inspiring discussions regarding the theory of higher Segal spaces in general. In particular, his proposal of corepresenting the S-construction via a cocyclic object was a key idea in this project. We would further like to thank A. Goncharov, and M. Kontsevich for important conversations which influenced the direction of this work. Finally, we would like to acknowledge a conversation involving D. Ben-Zvi, J. Lurie, D. Nadler, and A. Preygel, in which ideas related to this work were discussed. The work of T.D. was supported by a Simons Postdoctoral Fellowship. The work of M.K. was partially supported by an NSF grant and parts of it were carried out during visits to the Max-Planck-Institut für Mathematik in Bonn and to Université Paris-13, whose hospitality and financial support are gratefully acknowledged. 


\section{Background on the homotopy theory of dg-structures}

\subsection{Model structures on the category of differential $\mathbb{Z}$-graded categories.}

Let $\mathbf{k}$ be a field and $\operatorname{Vect}_{\mathbf{k}}^{\mathbb{Z}}$ be the category of $\mathbb{Z}$-graded $\mathbf{k}$-vector spaces. We denote by $\Sigma^{n}, n \in \mathbb{Z}$, the functor of shift of grading: $\left(\Sigma^{n} V\right)^{i}=V^{i+n}$. We denote by $\operatorname{Mod}_{\mathbf{k}}$ the category of cochain complexes of $\mathbf{k}$-vector spaces. The usual tensor product of complexes makes $\operatorname{Mod}_{\mathbf{k}}$ into a symmetric monoidal category, and the shift functor $\Sigma$ is defined by $\Sigma\left(V^{\bullet}\right)=$ $\Sigma(\mathbf{k}) \otimes V^{\bullet}$, where $\Sigma(\mathbf{k})$ is the vector space $\mathbf{k}$ in degree $(-1)$ with zero differential. By a $(\mathbb{Z}$-graded) $d g$-category we will mean a category $\mathcal{A}$ enriched in the symmetric monoidal category $\operatorname{Mod}_{\mathbf{k}}$. Note that $\operatorname{Mod}_{\mathbf{k}}$ itself is a dg-category. We denote by dgcat the category formed by small $\mathbf{k}$-linear dg-categories and their dg-functors. The category dgcat has a symmetric monoidal structure $\otimes$ given by the tensor product $\mathcal{A} \otimes \mathcal{B}$ of dg-categories $\mathcal{A}$ and $\mathcal{B}$ :

$$
\begin{aligned}
\mathrm{Ob}(\mathcal{A} \otimes \mathcal{B}) & =\mathrm{Ob}(\mathcal{A}) \times \mathrm{Ob}(\mathcal{B}), \\
\operatorname{Hom}_{\mathcal{A} \otimes \mathcal{B}}\left((x, y),\left(x^{\prime}, y^{\prime}\right)\right) & =\operatorname{Hom}_{\mathcal{A}}^{\bullet}\left(x, x^{\prime}\right) \otimes_{\mathbf{k}} \operatorname{Hom}_{\mathcal{B}}\left(y, y^{\prime}\right) .
\end{aligned}
$$

Recall that for dg-categories $\mathcal{A}, \mathcal{B}$ the category $\underline{\operatorname{Hom}}(\mathcal{A}, \mathcal{B})$ of dg-functors $\mathcal{A} \rightarrow \mathcal{B}$ is naturally a dg-category so that we have an adjunction

$$
\operatorname{Hom}_{\text {dgcat }}(\mathcal{A}, \underline{\operatorname{Hom}}(\mathcal{B}, \mathcal{C})) \cong \operatorname{Hom}_{\text {dgcat }}(\mathcal{A} \otimes \mathcal{B}, \mathcal{C}) .
$$

Definition 1.1.1. A dg-functor $f: \mathcal{A} \rightarrow \mathcal{B}$ of dg-categories is called:

- fully faithful, resp. quasi-fully faithful, if for any $x, y \in \mathrm{Ob}(\mathcal{A})$ the morphism of complexes

$$
f_{x, y}: \operatorname{Hom}_{\mathcal{A}}^{\bullet}(x, y) \longrightarrow \operatorname{Hom}_{\mathcal{B}}(f(x), f(y))
$$

is an isomorphism, resp. a quasi-isomorphism.

- a quasi-isomorphism, resp. quasi-equivalence, if $H^{\bullet}(f): H^{\bullet}(\mathcal{A}) \rightarrow H^{\bullet}(\mathcal{B})$ is an isomorphism, resp. an equivalence of graded $\mathbf{k}$-linear categories.

Dg-functors $\mathcal{A}^{\mathrm{op}} \rightarrow \operatorname{Mod}_{\mathbf{k}}$ will be called (right) $d g$-modules over $\mathcal{A}$, and the dg-category formed by them will be denoted $\operatorname{Mod}_{\mathcal{A}}$. We will use the $\mathrm{dg}$-version of the Yoneda embedding

$$
\Upsilon_{\mathcal{A}}: \mathcal{A} \longrightarrow \operatorname{Mod}_{\mathcal{A}}
$$

which is a fully faithful dg-functor. For background on model categories, see, e.g., Hov99], [DHKS04 and Appendix A to Lur09a. The category dgcat carries two model structures introduced by Tabuada [Tab07]. The first one, which we call the quasi-equivalence model structure, is characterized as follows:

(QW) Weak equivalences are quasi-equivalences. 
(QF) Fibrations are dg-functors $f: \mathcal{A} \rightarrow \mathcal{B}$ such that:

(1) $f$ is surjective on Hom-complexes.

(2) For any $x \in \operatorname{Ob}(\mathcal{A})$ and any homotopy equivalence $v: f(x) \rightarrow z$ in $\mathcal{B}$, there is a homotopy equivalence $u: x \rightarrow y$ in $\mathcal{A}$ such that $f(u)=v$ (in particular, $f(y)=x)$.

(QC) Cofibrations are defined by the left lifting property with respect to trivial fibrations.

The initial object in dgcat is the empty dg-category $\varnothing$ (no objects). The final object is the zero dg-category $\mathbf{0}$ with one object pt and $\mathrm{Hom}^{\bullet}(\mathrm{pt}, \mathrm{pt})=0$. Note that $(\mathrm{QF})$ implies that every dg-category is fibrant. Let $\mathrm{Qe}$ be the class of quasi-equivalences in dgcat, and let

$$
\text { Hqe }=\operatorname{dgcat}\left[\mathrm{Qe}^{-1}\right]
$$

denote the homotopy category of the quasi-equivalence model structure.

It follows from the results of Toën [Toë07] that $\otimes$ defines a closed symmetric monoidal structure on Hqe, so that we have dg-categories $R \underline{\operatorname{Hom}}(\mathcal{A}, \mathcal{B})$ together with natural isomorphisms (in Hqe)

$$
\operatorname{Hom}_{\mathrm{Hqe}}(\mathcal{A} \otimes \mathcal{B}, \mathcal{C}) \cong \operatorname{Hom}_{\mathrm{Hqe}}(\mathcal{A}, R \underline{\operatorname{Hom}}(\mathcal{B}, \mathcal{C})) .
$$

More precisely, Toën considers the situation when $\mathbf{k}$ is allowed to be an arbitrary commutative ring and uses $\otimes^{L}$, the derived functor of $\otimes$. In our case when $\mathbf{k}$ is a field, $\otimes$ preserves quasi-equivalences and hence does not need to be derived. Note that the internal Hom is not obtained as a derived functor, in the sense of model categories, of the bifunctor $\operatorname{Hom}(\mathcal{A}, \mathcal{B})$, since the latter does not take quasi-equivalences of cofibrant dg-categories into quasi-equivalences (see loc. cit. p.631). By the main result of [Toë07], the dg-category $R \underline{\operatorname{Hom}}(\mathcal{A}, \mathcal{B})$ can be explicitly described as the full dg-subcategory of $\operatorname{Mod}_{\mathcal{A}^{\mathrm{op} \otimes \mathcal{B}}}$ formed by those $\mathrm{dg}$-modules $M$ satisfying:

(1) $M$ is cofibrant.

(2) $M$ is right quasi-representable, i.e., for each $x \in \mathrm{Ob}(\mathcal{A})$ the right dg-module

$$
M(x,-): \mathcal{B}^{\text {op }} \longrightarrow \operatorname{Mod}_{\mathbf{k}}, \quad y \mapsto M(x, y)
$$

is quasi-isomorphic to a representable dg-module $\Upsilon_{\mathcal{B}}(f(x))$ for some object $f(x) \in \mathcal{B}$.

Recall that, as any model category, dgcat is equipped with simplicial mapping spaces $\operatorname{Map}(\mathcal{A}, \mathcal{B})$, obtained by Dwyer-Kan localization. However, as dgcat is not known to carry a simplicial model structure in the sense of [Hov99], the computation of $\operatorname{Map}(\mathcal{A}, \mathcal{B})$ is nontrivial. It was shown in [Toë07] that the mapping spaces can be computed via simplicial framings, leading to the explicit formula

$$
\operatorname{Map}(\mathcal{A}, \mathcal{B}) \simeq N\left(\operatorname{Mod}_{\mathcal{A}^{\mathrm{op} \otimes \mathcal{B}}}^{\mathrm{rqr}}, \mathrm{W}\right),
$$


the nerve of the category formed by all right quasi-representable $\mathcal{A}^{\mathrm{op}} \otimes \mathcal{B}$-modules and their weak equivalences. The isomorphisms 1.1 .3 can then be refined to the adjunction

$$
\operatorname{Map}(\mathcal{A} \otimes \mathcal{B}, \mathcal{C}) \simeq \operatorname{Map}(\mathcal{A}, R \underline{\operatorname{Hom}}(\mathcal{B}, \mathcal{C}))
$$

of simplicial mapping spaces (see [Toё07]).

Let $x, z$ be objects of a dg-category $\mathcal{A}$, and $m \in \mathbb{Z}$. We say that $z$ is realized as an $m$-fold shift of $x$, and write $z \simeq \Sigma^{m} x$, if we are given an isomorphism of dg-functors

$$
\operatorname{Hom}^{\bullet}(-, z) \longrightarrow \Sigma^{m} \operatorname{Hom}^{\bullet}(-, x), \quad \mathcal{A}^{\mathrm{op}} \rightarrow C_{\mathbf{k}} .
$$

Note that $\Sigma^{m} x$, if exists, is defined uniquely up to a unique isomorphism.

We recall (e.g., [TV07]) that $\operatorname{Mod}_{\mathcal{A}}$, equipped with the projective model structure, is a $\operatorname{Mod}_{\mathbf{k}}$-enriched model category in which weak equivalences are quasi-isomorphisms of dgmodules, and all objects are fibrant. We denote by $\operatorname{Mod}_{\mathcal{A}}^{\circ} \subset \operatorname{Mod}_{\mathcal{A}}$ the full dg-subcategory of cofibrant (and automatically fibrant) objects. We also denote by

$$
D(\mathcal{A})=H^{0}\left(\operatorname{Mod}_{\mathcal{A}}\right)=\operatorname{Mod}_{\mathcal{A}}\left[\mathrm{Qis}^{-1}\right]
$$

the homotopy category of $\operatorname{Mod}_{\mathcal{A}}$, which is commonly called the derived category of $\mathcal{A}$. Thus, we have an equivalence

$$
D(\mathcal{A}) \simeq H^{0}\left(\operatorname{Mod}_{\mathcal{A}}^{\circ}\right)
$$

This can be rephrased by saying that we have natural complexes $R \operatorname{Hom}_{\mathcal{A}}(M, N)$, given for each $M, N \in \operatorname{Mod}_{\mathcal{A}}$ and satisfying

$$
H^{i} R \operatorname{Hom}_{\mathcal{A}}^{\bullet}(M, N) \cong \operatorname{Hom}_{D(\mathcal{A})}\left(M, \Sigma^{i} N\right) .
$$

We further recall that a dg-module $M \in \operatorname{Mod}_{\mathcal{A}}$ is called perfect, if $M$ is compact in $D(\mathcal{A})$ in the categorical sense, i.e., the functor $\operatorname{Hom}_{D(\mathcal{A})}(M,-)$ commutes with infinite direct sums. We denote by $\operatorname{Perf}_{\mathcal{A}}$ the dg-category whose objects are perfect dg-modules and

$$
\operatorname{Hom}_{\operatorname{Perf} \mathcal{A}}^{\bullet}(M, N)=R \operatorname{Hom}_{\mathcal{A}}^{\bullet}(M, N) .
$$

Any dg-functor $\mathcal{A} \rightarrow \mathcal{B}$ gives rise to a Quillen adjunction

$$
f_{!}: \operatorname{Mod}_{\mathcal{A}} \longleftrightarrow \operatorname{Mod}_{\mathcal{B}}: f^{*}
$$

where $f^{*}$ is obtained by composing dg-functors $\mathcal{B}^{\text {op }} \rightarrow \operatorname{Mod}_{\mathbf{k}}$ with $f^{\mathrm{op}}$. This induces a dg-functor

$$
f_{!}: \operatorname{Perf}_{\mathcal{A}} \rightarrow \operatorname{Perf}_{\mathcal{B}}
$$

and a triangulated functor

$$
f^{*}: D(\mathcal{B}) \rightarrow D(\mathcal{A}) .
$$

The dg-Yoneda embedding factors through a faithful dg-embedding

$$
\beta_{\mathcal{A}}: \mathcal{A} \longrightarrow \operatorname{Perf}_{\mathcal{A}}
$$


Definition 1.1.5. A dg-category $\mathcal{A}$ is called perfect, if $\beta_{\mathcal{A}}$ is a quasi-equivalence.

It is known that for a perfect $\mathcal{A}$, we have that $H^{0}(\mathcal{A})$ is an idempotent-complete triangulated category.

We now define a second model structure on dgcat which we call the Morita model structure. First, we recall that a dg-functor $f: \mathcal{A} \rightarrow \mathcal{B}$ with $\mathcal{A} \neq \varnothing$ is called a Morita equivalence, if the following equivalent conditions are satisfied:

(1) $f_{!}: \operatorname{Perf}_{\mathcal{A}} \rightarrow \operatorname{Perf}_{\mathcal{B}}$ is a dg-equivalence of dg-categories.

(2) $f^{*}: D(\mathcal{B}) \rightarrow D(\mathcal{A})$ is an equivalence of triangulated categories.

See [Kel94] and [Tab07], §2.5, for details, including the treatment of the case when $\mathcal{A}$ is empty. The Morita model structure on dgcat is defined by ([Tab07], Th. 0.7):

(MW) Weak equivalences are Morita equivalences.

(MC) Cofibrations are the same as for the quasi-equivalence model structure.

(MF) Fibrations are determined by the right lifting property with respect to trivial cofibrations.

We denote by Mo the class of Morita equivalences and by $\mathrm{Hmo}=\operatorname{dgcat}\left[\mathrm{Mo}^{-1}\right]$ the homotopy category of the Morita model structure. There is a Quillen adjunction

$$
\text { id : (dgcat, Qe }) \longleftrightarrow(\text { dgcat, Mo }): \text { id }
$$

which exhibits the Morita model structure on dgcat as a left Bousfield localization (see [Hir03]) of the quasi-equivalence model structure and hence induces an adjunction of homotopy categories

$$
F: \mathrm{Hqe} \longleftrightarrow \mathrm{Hmo}: G
$$

where $G$ is fully faithful.

Proposition 1.1.6. (a) A dg-category is fibrant for the Morita model structure, if and only if it is perfect.

(b) For any dg-category $\mathcal{A}$ the canonical dg-functor

$$
\beta_{\operatorname{Perf}_{\mathcal{A}}}: \operatorname{Perf}_{\mathcal{A}} \longrightarrow \operatorname{Perf}_{\operatorname{Perf}_{\mathcal{A}}}
$$

is a quasi-equivalence. In particular, $\operatorname{Perf}_{\mathcal{A}}$ is perfect, and $\beta_{\mathcal{A}}: \mathcal{A} \rightarrow \operatorname{Perf}_{\mathcal{A}}$ is a Morita equivalence.

Proof. (a) is Proposition 0.9 of [Tab07]. Part (b) is Lemma 7.5 in [Toë07]

Note that, as a consequence, we obtain that the Yoneda embedding $\beta_{\mathcal{A}}: \mathcal{A} \rightarrow \operatorname{Perf}_{\mathcal{A}}$ exhibits $\operatorname{Perf}_{\mathcal{A}}$ as a fibrant replacement of $\mathcal{A}$ in the Morita model structure. 
Example 1.1.7 (Morita duality). Considering $\mathbf{k}$ as a 1-object dg-category, we see that $\operatorname{Perf}_{\mathbf{k}} \subset \operatorname{Mod}_{\mathbf{k}}$ is the full dg-subcategory of complexes with total cohomology space finitedimensional. By the above, it is a Morita fibrant replacement of $\mathbf{k}$. The derived tensor product $\otimes^{L}$ makes Hmo into a symmetric monodical category with unit object $\mathbf{k}$. This monoidal structure is closed, with internal Hom objects given by

$$
R \underline{\operatorname{Hom}}_{\text {Hmo }}(\mathcal{A}, \mathcal{B})=R \underline{\operatorname{Hom}}\left(\mathcal{A}, \operatorname{Perf}_{\mathcal{B}}\right)
$$

(fibrant replacement of the second argument), see [Tab07], Cor. 0.12. Accordingly, for a dg-category $\mathcal{A}$, we will call

$$
\mathcal{A}^{\vee}=R \underline{\operatorname{Hom}}\left(\mathcal{A}, \operatorname{Perf}_{\mathbf{k}}\right)
$$

the Morita dual of $\mathcal{A}$. By the computation of $R \underline{\mathrm{Hom}}$ in [Toё07], the dg-category $\mathcal{A}^{\vee}$ is identified with the full dg-subcategory in $\operatorname{Mod}_{\mathcal{A}^{\text {op }}}$ formed by $\mathrm{dg}$-modules $M$, which are cofibrant and pseudo-perfect, i.e., such that each $M(x)$ is a perfect complex.

Note that passing to the dual object is a contravariant functor

$$
(-)^{\vee}: \mathrm{Hmo}^{\mathrm{op}} \longrightarrow \mathrm{Hmo}
$$

As in any closed monoidal category, we say that a dg-category $\mathcal{A}$ is dualizable in $\mathrm{Hmo}$, if the canonical dg-functor

$$
\mathcal{A}^{\vee} \otimes \mathcal{B} \longrightarrow R \underline{\operatorname{Hom}}_{\mathrm{Hmo}}(\mathcal{A}, \mathcal{B})
$$

is a Morita equivalence for any $\mathcal{B}$.

Definition 1.1.9. A dg-category $\mathcal{A}$ is called

- proper, if each complex $\operatorname{Hom}_{\mathcal{A}}^{\bullet}(x, y)$ belongs to $\operatorname{Perf}_{\mathbf{k}}$.

- smooth, if the diagonal $\mathcal{A}^{\mathrm{op}} \otimes \mathcal{A}$-module

$$
\mathcal{A}:(x, y) \mapsto \operatorname{Hom}_{\mathcal{A}}^{\bullet}(x, y)
$$

belongs to $\operatorname{Perf}_{\mathcal{A}^{\mathrm{o}} \otimes \mathcal{A}}$.

We recall the following result from [TV07].

Proposition 1.1.10. A dg-category $\mathcal{A}$ is dualizable in $\mathrm{Hmo}$, if and only if it is smooth and proper. In this case a dg-module over $\mathcal{A}$ is perfect if and only if it is pseudo-perfect, and so

$$
\mathcal{A}^{\vee} \simeq \operatorname{Perf}_{\mathcal{A}^{\mathrm{op}}}
$$

is Morita equivalent to $\mathcal{A}^{\mathrm{op}}$.

Proof. Lemma 2.8 in [TV07. 


\subsection{The 2-periodic case}

Let $\operatorname{Vect}_{\mathbf{k}}^{(2)}$, resp. $\operatorname{Mod}_{\mathbf{k}}^{(2)}$, be the category of $\mathbb{Z} / 2 \mathbb{Z}$-graded $\mathbf{k}$-vector spaces, resp. cochain complexes, equipped with the $\mathbb{Z} / 2 \mathbb{Z}$-graded tensor product. The functor $\Sigma$ of shift of grading on these categories satisfies $\Sigma^{2}=\mathrm{Id}$.

We have an obvious $\mathbb{Z} / 2 \mathbb{Z}$-graded analogue of the concept of a dg-category: a small category enriched over $\operatorname{Mod}_{\mathbf{k}}^{(2)}$. We refer to these structures as 2-periodic, or $\mathbb{Z} / 2 \mathbb{Z}$-graded, dg-categories and will leave out the extra adjective when it is obvious from the context. We will denote by dgcat ${ }^{(2)}$ the category of $\mathbb{Z} / 2 \mathbb{Z}$-graded dg-categories and their dg-functors.

All the aspects of the homotopy theory of dg-categories and their dg-modules, as recalled in \$1.1, can be extended to the $\mathbb{Z} / 2 \mathbb{Z}$-graded case without any substantial changes. A convenient way to compare to the $\mathbb{Z}$-graded theory of $\$ 1.1$ is as follows Dyc11, §5.1]. Note that objects of $\operatorname{Mod}_{\mathbf{k}}^{(2)}$ can be seen as 2-periodic $\mathbb{Z}$-graded cochain complexes over $\mathbf{k}$, i.e., as dg-modules over the commutative dg-algebra

$$
\mathbf{k}\left[u, u^{-1}\right], \quad \operatorname{deg}(u)=2, d u=0 .
$$

Under this identification, the $\mathbb{Z} / 2 \mathbb{Z}$-graded tensor product corresponds to $\otimes_{\mathbf{k}\left[u^{ \pm 1}\right]}$. We have an adjunction

$$
P: \operatorname{Mod}_{\mathbf{k}} \longleftrightarrow \operatorname{Mod}_{\mathbf{k}\left[u^{ \pm 1}\right]}=\operatorname{Mod}_{\mathbf{k}}^{(2)}: F
$$

where $F$ is the forgetful functor, and $P$ is the functor of 2-periodization given by

$$
P\left(V^{\bullet}\right)=V^{\bullet} \otimes_{\mathbf{k}} \mathbf{k}\left[u^{ \pm 1}\right], \quad P\left(V^{\bullet}\right)^{\bar{i}}=\bigoplus_{i \equiv \bar{i} \bmod 2} V^{i}, \bar{i} \in \mathbb{Z} / 2 \mathbb{Z} .
$$

As explained in loc. cit., this is a Quillen adjunction of model categories. Applying this adjunction on the level of Hom-complexes, we get an adjunction

$$
P: \text { dgcat } \longleftrightarrow \operatorname{dgcat}^{(2)}: F
$$

The quasi-equivalence model structure on $\mathrm{dgcat}^{(2)}$ is defined by:

$\left(\mathrm{QW}^{(2)}\right)$ Weak equivalences are quasi-equivalences, i.e., morphisms taken by $F$ into quasiequivalences in dgcat.

$\left(\mathrm{QF}^{(2)}\right)$ Fibrations are defined by the right lifting property with respect to the set of generating trivial cofibrations that is obtained by applying $P$ to the generating set in Tab07]. This leads to the description of fibrations which is the $\mathbb{Z} / 2 \mathbb{Z}$-graded version of $(\mathrm{QF})$.

$\left(\mathrm{QC}^{(2)}\right)$ Cofibrations are defined by the left lifting property with respect to trivial fibrations.

As observed in [Dyc11, §5.1], this indeed defines a model structure such that (1.2.2) becomes a Quillen adjunction. We denote by $\mathrm{Hqe}^{(2)}$ the homotopy category of this model structure.

All results and definitions recalled in $\$ 1.1$ have obvious 2-periodic case analogues. In particular, we will denote by $\mathrm{Hmo}^{(2)}$ the Morita homotopy category of $\mathrm{dgcat}^{(2)}$ and will refer to 2-periodic versions of other statements in $\$ 1.1$ without further explanation. 


\section{Loop factorizations}

\section{$2.1 \quad \mathbb{Z}_{+}$-categories and the cyclic category}

Definition 2.1.1. By a $\mathbb{Z}_{+}$-category we mean a pair $(\mathcal{C}, w)$, where $\mathcal{C}$ is a category and $w: \operatorname{Id}_{\mathcal{C}} \Rightarrow \mathrm{Id}_{\mathcal{C}}$ is a natural transformation. Thus $w$ is a system of morphisms $w_{x}: x \rightarrow x$ given for each $x \in \mathrm{Ob}(\mathcal{C})$ and such that $f w_{x}=w_{y} f$ for each morphism $f: x \rightarrow y$. If $w$ is clear from the context, we will omit it from the notation.

This definition is due to Drinfeld [Dr04]. Here are two reformulations. First, let us denote by $\mathbb{Z}_{+}=\{0,1,2, \ldots\}$ the additive monoid of non-negative integers, and let $B \mathbb{Z}_{+}$be the category with one object corresponding to $\mathbb{Z}_{+}$. Since $\mathbb{Z}_{+}$is commutative, $B \mathbb{Z}_{+}$is a symmetric monoidal category. $\mathrm{A} \mathbb{Z}_{+}$-category is the same as a category with action of $B \mathbb{Z}_{+}$.

Second, let $\mathbb{Z}_{+}-\mathcal{S}$ et be the category of $\mathbb{Z}_{+}$-sets, i.e., of sets with a $\mathbb{Z}_{+}$-action. Given $\mathbb{Z}_{+}$-sets $A$ and $B$, we define the $\mathbb{Z}_{+}$-set

$$
A \otimes_{\mathbb{Z}_{+}} B=A \times B /\left\{(n+a, b) \sim(a, n+b), n \in \mathbb{Z}_{+}\right\} .
$$

This operation makes $\mathbb{Z}_{+}$-Set into a symmetric monoidal category, with unit object $\mathbb{Z}_{+}$ (considered as an $\mathbb{Z}_{+}$-set). Thus a morphism of $\mathbb{Z}_{+}$-sets $A \otimes_{\mathbb{Z}_{+}} B \rightarrow C$ is the same as an $\mathbb{Z}_{+}$-bilinear map $A \times B \rightarrow C$.

Proposition 2.1.2. Let $\mathcal{C}$ be a small category. The two following sets of data are in bijection:

(1) Structures of a $\mathbb{Z}_{+}$-category on $\mathcal{C}$, i.e., natural transformations $w: \operatorname{Id}_{\mathcal{C}} \Rightarrow \operatorname{Id}_{\mathcal{C}}$.

(2) Enrichments of $\mathcal{C}$ in $\mathbb{Z}_{+}-\mathcal{S} e t$, i.e., ways of defining $\mathbb{Z}_{+}$-action on each $\operatorname{Hom}_{\mathcal{C}}(x, y)$ so that the composition is $\mathbb{Z}_{+}$-bilinear.

Proof. Given $w$, we define, for any $f: x \rightarrow y$, the morphism $n+f$ as $f w_{x}^{n}=w_{y}^{n} f$. Given an enrichment, i.e., system of actions of $\mathbb{Z}_{+}$on each $\operatorname{Hom}_{\mathcal{C}}(x, y)$, we define $w_{x}=1+\operatorname{Id}_{x}$. The details are left to the reader.

Example 2.1.3. Let $n \geq 0$ and consider the circular quiver $Q^{n}$ with set of vertices given by $\mathbb{Z} /(n+1)$ and, for every $i \in \mathbb{Z} /(n+1)$, an arrow from $i$ to $i+1$. Let $Q^{n}$ be the category freely generated by $Q^{n}$. Thus $Q^{0}=B\left(\mathbb{Z}_{+}\right)$. The category $Q^{n}$ is admits a natural $\mathbb{Z}_{+}$-category structure where $w_{i}: i \rightarrow i$ is the cycle of degree 1 at $i$.

We define an $\mathbb{Z}_{+}$-functor between $\mathbb{Z}_{+}$-categories as an enriched functor. Explicitly, if we write our $\mathbb{Z}_{+}$-categories as $(\mathcal{C}, w)$ and $\left(\mathfrak{C}^{\prime}, w^{\prime}\right)$, then a $\mathbb{Z}_{+}$-functor between them is an ordinary functor $F: \mathcal{C} \rightarrow \mathcal{C}^{\prime}$ such that $F\left(w_{x}\right)=w_{F(x)}^{\prime}$ for each $x \in \mathrm{Ob}(\mathcal{C})$. The set of $\mathbb{Z}_{+}$-functors between $\mathbb{Z}_{+}$-categories $\mathcal{C}$ and $\mathcal{C}^{\prime}$ is denoted by $\operatorname{Fun}_{\mathbb{Z}_{+}}\left(\mathcal{C}, \mathcal{C}^{\prime}\right)$. We denote by $\mathbb{Z}_{+}$-Cat the category of $\mathbb{Z}_{+}$-categories with morphisms given by $\mathbb{Z}_{+}$-functors. 
Further, $\operatorname{Fun}_{\mathbb{Z}_{+}}\left(\mathcal{C}, \mathrm{C}^{\prime}\right)$ is the set of objects of a category $\underline{\operatorname{Fun}}_{\mathbb{Z}_{+}}\left(\mathcal{C}, \mathrm{C}^{\prime}\right)$ whose morphisms are natural transformations $\eta: F \rightarrow G$ of $\mathbb{Z}_{+}$-functors. Note that $\underline{\mathrm{Fun}}_{\mathbb{Z}_{+}}\left(\mathrm{C}, \mathrm{C}^{\prime}\right)$ itself carries a structure of a $\mathbb{Z}_{+}$-category: for $\eta$ as above we define $(n+\eta)_{x}=n+\eta_{x}: F(x) \rightarrow G(x)$ for $x \in \mathrm{Ob}(\mathcal{C})$ and $n \in \mathbb{Z}_{+}$.

Given $\mathbb{Z}_{+}$-categories $\mathcal{C}$ and $\mathcal{C}^{\prime}$, we define a $\mathbb{Z}_{+}$-category $\mathcal{C} \otimes_{\mathbb{Z}_{+}} \mathcal{C}^{\prime}$ with set of objects $\mathrm{Ob}(\mathrm{C}) \times \mathrm{Ob}(\mathcal{C})$ and morphisms given by

$$
\operatorname{Hom}_{\mathcal{C}_{\mathbb{Z}_{+}} \mathrm{e}^{\prime}}\left(\left(x, x^{\prime}\right),\left(y, y^{\prime}\right)\right):=\operatorname{Hom}_{\mathcal{C}}(x, y) \otimes_{\mathbb{Z}_{+}} \operatorname{Hom}_{\mathcal{C}^{\prime}}\left(x^{\prime}, y^{\prime}\right)
$$

where $x, y$ and $x^{\prime}, y^{\prime}$ are objects of $\mathcal{C}$ and $\mathcal{C}^{\prime}$, respectively. This operation provides a monoidal structure on $\mathbb{Z}_{+}$- at with unit given by the $\mathbb{Z}_{+}$-category $Q^{0}$. We have an adjunction

$$
\operatorname{Fun}_{\mathbb{Z}_{+}}\left(\mathcal{C} \otimes_{\mathbb{Z}_{+}} \mathcal{C}^{\prime}, \mathcal{D}\right) \cong \operatorname{Fun}_{\mathbb{Z}_{+}}\left(\mathcal{C}, \underline{\operatorname{Fun}}_{\mathbb{Z}_{+}}\left(\mathcal{C}^{\prime}, \mathcal{D}\right)\right)
$$

which shows that the monoidal structure on $\mathbb{Z}_{+}-\mathrm{C} a t$ is closed.

(2.1.4) The cyclic category, cyclic ordinals and cyclic objects. We recall Connes' definition of the cyclic category $\Lambda$, see Con94]. The objects of $\Lambda$ are given by nonnegative integers where we denote the object corresponding to $n \geq 0$ by $\langle n\rangle$. We use the map

$$
\mathbb{Z} /(n+1) \rightarrow \mathbb{C}, \quad k \mapsto \exp \left(\frac{2 \pi i k}{n+1}\right)
$$

to identify the elements of $\mathbb{Z} /(n+1)$ with the set of $(n+1)$ st roots of unity contained in the unit circle $S^{1} \subset \mathbb{C}$. A map $f:\langle m\rangle \rightarrow\langle n\rangle$ in $\Lambda$ is given by a homotopy class of continuous maps $f: S^{1} \rightarrow S^{1}$ of degree 1 mapping $\mathbb{Z} /(m+1)$ into $\mathbb{Z} /(n+1)$.

Following Drinfeld [Dr04], we provide an alternative description of $\Lambda$.

Proposition 2.1.6. There is a fully faithful functor

$$
\mathrm{FC}: \Lambda \longrightarrow \mathbb{Z}_{+}-\mathrm{e} a t, \quad\langle n\rangle \mapsto Q^{n}
$$

which embeds $\Lambda$ into the category of $\mathbb{Z}_{+}$-categories.

More generally, by a (total) cyclic order on a finite set $I,|I|=n+1>0$, we mean a class of total orders up to the action of the group $\mathbb{Z} /(n+1)$ of cyclic rotations. Alternatively, a cyclic order can be defined as a ternary relation of a certain kind [Hun16, STZ11]. A finite set with a cyclic order will be called a finite cyclic ordinal. Each finite cyclic ordinal $I$ is isomorphic to some $\mathbb{Z} /(n+1)$ and so gives rise to a $\mathbb{Z}_{+}$-category $\mathcal{Q}^{I}$ as in Example 2.1.3. We will sometimes replace $\Lambda$ by an equivalent large category $\Lambda$ whose objects are all finite cyclic ordinals and

$$
\operatorname{Hom}_{\Lambda}(I, J):=\operatorname{Hom}_{\mathbb{Z}_{+}-\mathrm{Cat}}\left(Q^{I}, Q^{J}\right) .
$$

By a cyclic (resp.cocyclic) object in a category $\mathbf{C}$, we mean a contravariant (reap. covariant) functor $X: \Lambda \rightarrow \mathrm{C}$. Note that such functor canonically extends to $\Lambda$, so we can speak about objects $X(I) \in \mathbf{C}$ for any finite cyclic ordinal $I$. 


\subsection{Loop factorizations in $\mathbb{Z}_{+}$-categories}

Let $(\mathcal{C}, w)$ (or simply $\mathcal{C}$ ) be an $\mathbb{Z}_{+}$-category. We define a loop factorization in $\mathcal{C}$ to be a functor of $\mathbb{Z}_{+}$-categories $F: Q^{1} \rightarrow \mathcal{C}$. Explicitly, a loop factorization can be viewed a datum

$$
F=\left\{x_{1} \stackrel{\psi}{\stackrel{\psi}{\longrightarrow}} x_{0}\right\}
$$

of objects and morphisms in $\mathcal{C}$ such that $\varphi \psi=w_{x_{0}}$ and $\psi \varphi=w_{x_{1}}$.

Example 2.2.1. Let $R$ be an associative ring, and $w \in R$ be a central element. Let $\operatorname{Perf}_{R}$ be the category of finitely generated projective left $R$-modules. Multiplication by $w$ makes $\operatorname{Perf}_{R}$ into a $\mathbb{Z}_{+}$-category. A loop factorization in $\operatorname{Perf}_{R}$ is the same as a matrix factorization of $w$ in the standard sense [Eis80]. Further, the construction of dg-categories of matrix factorization extends to our context as follows.

Let $\mathbf{k}$ be a field and $\mathcal{C}$ be a $\mathbb{Z}_{+}$-category. Denote by $\mathbf{k}[\mathcal{C}]$ the $\mathbf{k}$-linear envelope of $\mathcal{C}$, i.e., the category with the same objects as $\mathcal{C}$ and $\operatorname{Hom}_{\mathbf{k}[\mathfrak{e}]}(x, y)$ being the $\mathbf{k}$-vector space spanned by the set $\operatorname{Hom}_{\mathfrak{C}}(x, y)$. For any two loop factorizations: $F$ as above and $F^{\prime}=\left\{x_{1}^{\prime} \underset{\varphi^{\prime}}{\stackrel{\psi^{\prime}}{\leftrightarrows}} x_{0}^{\prime}\right\}$ in $\mathcal{C}$, we define the $\mathbb{Z} / 2 \mathbb{Z}$-graded $\mathbf{k}$-module $\operatorname{Hom} \bullet\left(F, F^{\prime}\right)$ given by

$$
\begin{aligned}
& \operatorname{Hom}^{0}\left(F, F^{\prime}\right)=\operatorname{Hom}_{\mathbf{k}[\mathrm{e}]}\left(x_{0}, x_{0}^{\prime}\right) \oplus \operatorname{Hom}_{\mathbf{k}[\mathrm{e}]}\left(x_{1}, x_{1}^{\prime}\right) \\
& \operatorname{Hom}^{1}\left(F, F^{\prime}\right)=\operatorname{Hom}_{\mathbf{k}[\mathrm{e}]}\left(x_{1}, x_{0}^{\prime}\right) \oplus \operatorname{Hom}_{\mathbf{k}[\mathrm{e}]}\left(x_{0}, x_{1}^{\prime}\right)
\end{aligned}
$$

Any element of $\operatorname{Hom}^{\bullet}\left(F, F^{\prime}\right)$ can be represented by a matrix

$$
\left(\begin{array}{ll}
\alpha & \gamma \\
\delta & \beta
\end{array}\right)=\left(\begin{array}{ll}
\alpha & 0 \\
0 & \beta
\end{array}\right)+\left(\begin{array}{ll}
0 & \gamma \\
\delta & 0
\end{array}\right)
$$

with $(\alpha, \beta) \in \operatorname{Hom}^{0}\left(F, F^{\prime}\right)$ and $(\gamma, \delta) \in \operatorname{Hom}^{1}\left(F, F^{\prime}\right)$. We define the differential on $\operatorname{Hom}^{\bullet}\left(F, F^{\prime}\right)$ by the formulas

$$
\begin{aligned}
\left(\begin{array}{cc}
\alpha & 0 \\
0 & \beta
\end{array}\right) & \mapsto\left(\begin{array}{cc}
0 & \varphi^{\prime} \\
\psi^{\prime} & 0
\end{array}\right)\left(\begin{array}{cc}
\alpha & 0 \\
0 & \beta
\end{array}\right)-\left(\begin{array}{cc}
\alpha & 0 \\
0 & \beta
\end{array}\right)\left(\begin{array}{cc}
0 & \varphi \\
\psi & 0
\end{array}\right) \\
\left(\begin{array}{ll}
0 & \gamma \\
\delta & 0
\end{array}\right) & \mapsto\left(\begin{array}{cc}
0 & \varphi^{\prime} \\
\psi^{\prime} & 0
\end{array}\right)\left(\begin{array}{ll}
0 & \gamma \\
\delta & 0
\end{array}\right)+\left(\begin{array}{cc}
0 & \gamma \\
\delta & 0
\end{array}\right)\left(\begin{array}{cc}
0 & \varphi \\
\psi & 0
\end{array}\right)
\end{aligned}
$$

In this way we get a $\mathbb{Z} / 2 \mathbb{Z}$-graded dg-category, denoted $\operatorname{LF}(\mathcal{C})$, whose objects are loop factorizations in $\mathcal{C}$ and the Hom-complexes are given by the $\operatorname{Hom}^{\bullet}\left(F, F^{\prime}\right)$.

Theorem 2.2.4. Associating to a $\mathbb{Z}_{+}$-category $\mathcal{C}$ the $d g$ category $\mathrm{LF}(\mathcal{C})$, gives a functor

$$
\mathrm{LF}: \mathbb{Z}_{+}-\mathrm{Cat} \longrightarrow \operatorname{dgcat}^{(2)} \text {. }
$$

Proof. This follows directly from the definitions, since the formulas (2.2.2) and (2.2.3) are intrinsically functorial. 


\subsection{The cyclic model for $D^{(2)}\left(A_{n}\right.$ - mod $)$ via matrix factorizations}

(2.3.1) The root category and its cyclic symmetry. Consider the Dynkin quiver of type $A_{n}$ with its standard orientation:

$$
A_{n}=\left\{\stackrel{1}{\bullet} \stackrel{2}{\longrightarrow} \longrightarrow \ldots \longrightarrow{ }^{n}\right\} .
$$

Denote $A_{n}$-mod the category of finite-dimensional representations of $A_{n}$ over $\mathbf{k}$, i.e., of diagrams $V_{1} \rightarrow \cdots \rightarrow V_{n}$ of finite-dimensional $\mathbf{k}$-vector spaces, and let $D^{(2)}\left(A_{n}\right.$-mod) be the derived category of 2-periodic complexes over $A_{n}$-mod. The latter category is known as the root category for $A_{n}$ because its indecomposable objects are in bijection with roots of the root system of type $A_{n}$, see Hap87. More precisely, for each $1 \leq i \leq j \leq n$ we denote $\mathbf{k}_{[i, j]} \in A_{n}$-mod the indecomposable object having $\mathbf{k}$ in positions from the interval $[i, j]$ and 0 elsewhere. Then indecomposable objects in $D^{(2)}\left(A_{n}\right.$-mod) are

$$
\mathbf{e}_{i j}=\left\{\begin{array}{l}
\mathbf{k}_{[n-j+1, n-i]}, \text { if } i<j ; \\
\Sigma \mathbf{k}_{[n-i+1, n-j]}, \text { if } i>j,
\end{array}, \quad i, j \in\{0,1, \cdots, n\}, i \neq j,\right.
$$

so that $\Sigma \mathbf{e}_{i j} \simeq \mathbf{e}_{j i}$ in all cases. The Grothendieck group $K\left(D^{(2)}\left(A_{n}\right.\right.$-mod $\left.)\right)$ is identified with the root lattice for $A_{n}$, and the class of $\mathbf{e}_{i j}$ is the standard root $e_{i j}$.

Further, $D^{(2)}\left(A_{n}\right.$-mod) carries a self-equivalence known as the (derived) Coxeter functor

$$
C_{n}: D^{(2)}\left(A_{n} \text {-mod }\right) \longrightarrow D^{(2)}\left(A_{n}-\bmod \right), \quad C_{n}^{n+1} \simeq \text { Id } .
$$

It can be defined either as the composition of derived reflection functors [GM03], or characterized intrinsically by the condition that $\Sigma \circ C_{n}^{-1}$ is the Serre functor of $D^{(2)}\left(A_{n}\right.$-mod $)$, i.e., we have natural isomorphisms

$$
\operatorname{Hom}_{D^{(2)}\left(A_{n} \text {-mod }\right)}\left(V^{\bullet}, W^{\bullet}\right)^{*} \simeq \operatorname{Hom}_{D^{(2)}\left(A_{n} \text {-mod }\right)}\left(W^{\bullet}, \Sigma C_{n}^{-1}\left(V^{\bullet}\right)\right) .
$$

The automorphism of $K\left(D^{(2)}\left(A_{n}\right.\right.$-mod $\left.)\right)$ induced by $C_{n}$, is the Coxeter transformation $c_{n}$ from the Weyl group $W_{A_{n}}=S_{n+1}$. This transformation is the $(n+1)$-cycle: $c_{n}=(012 \cdots n)$. Being an equivalence, $C_{n}$ preserves indecomposable objects and the action on such objects corresponds to the action of $c_{n}$ on the roots. In particular, the action on simple modules is

$$
\mathbf{k}_{[n, n]} \longmapsto \mathbf{k}_{[n-1, n-1]} \longmapsto \cdots \longmapsto \mathbf{k}_{[1,1]} \longmapsto \Sigma \mathbf{k}_{[1, n]} .
$$

Example 2.3.4. Let $P_{n+1}$ be the convex plane $(n+1)$-gon with vertices labelled by $0,1, \cdots, n$ in the counterclockwise order. We can represent $\mathbf{e}_{i j}$ as an oriented arc (side or diagonal) in $P_{n}$ going from the vertex $i$ to the vertex $j$, so applying $\Sigma$ corresponds to change of orientation. We say that a triple of distinct elements $(i, j, k) \in\langle n\rangle \simeq \mathbb{Z} /(n+1)$ is in the counterclockwise cyclic order, if it can be brought by a cyclic rotation to a triple 
$\left(i^{\prime}, j^{\prime}, k^{\prime}\right)$ with $0 \leq i^{\prime}<j^{\prime}<k^{\prime} \leq n$. For any such triple we have a distinguished triangle in $D^{(2)}\left(A_{n}\right.$-mod):

$$
\mathbf{e}_{i j} \longrightarrow \mathbf{e}_{i k} \longrightarrow \mathbf{e}_{j k} \longrightarrow \Sigma \mathbf{e}_{i j}
$$

which can be depicted as a triangle inscribed into $P_{n+1}$, similarly to (0.1):

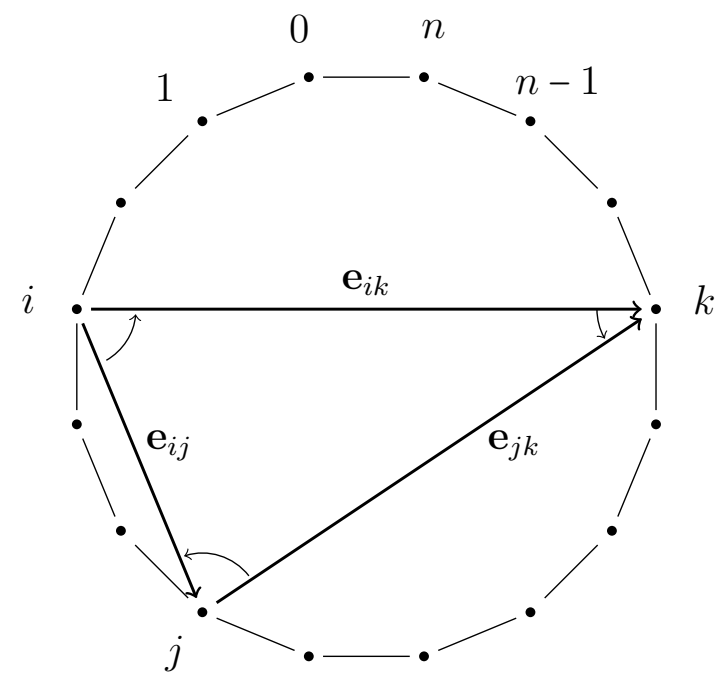

(2.3.5) Graded matrix factorizations. Let $L$ be an abelian group, and $R=\oplus_{a \in L} R_{a}$ be an $L$-graded associative $\mathbf{k}$-algebra (with $\mathbf{k} \subset R_{0}$ ). Let $w \in R_{0}$ be a central element, and $\operatorname{Perf}_{R}^{L}$ be the category of finitely generated projective $L$-graded left $R$-modules (and their morphisms of degree 0). As in Example 2.2.1, $\operatorname{Perf}_{R}^{L}$ is then a k-linear $\mathbb{Z}_{+}$-category. We define the dg-category $\operatorname{MF}^{L}(R, w)$ to have, as objects, loop factorizations in $\operatorname{Perf}_{R}^{L}$ and Hom-complexes defined analogously to (2.2.2) but with $\operatorname{Hom}_{\mathbf{k}[\mathrm{e}]}$, replaced by $\operatorname{Hom}_{\operatorname{Perr}_{R}^{L}}$. We call $\mathrm{MF}^{L}(R, w)$ the dg-category of $L$-graded matrix factorizations of $w$. As with any category of matrix factorizations, the dg-category $\operatorname{MF}^{L}(R, w)$ is perfect. In particular, the category $H^{0} \mathrm{MF}^{L}(R, w)$ is triangulated. For $i \in L$, and $M \in \operatorname{Perf}_{R}^{L}$, we denote by $M(i)$ the graded $R$-module with $M(i)_{n}=M_{i+n}$.

Let $L=\mathbb{Z} /(n+1)$ where $n \geq 0$. We consider the polynomial ring $R=\mathbf{k}[z]$ as an $L$-graded k-algebra with $\operatorname{deg}(z)=1$, and take $w=z^{n+1}$. We introduce the notation

$$
\mathcal{T}^{n}=\mathrm{MF}^{\mathbb{Z} /(n+1)}\left(\mathbf{k}[z], z^{n+1}\right) .
$$

The rank of an object $F=\left\{M_{1} \underset{\varphi}{\stackrel{\psi}{\rightleftarrows}} M_{0}\right\} \in \mathcal{T}^{n}$ is, by the definition, the rank of $M_{0}$ and $M_{1}$ as free $\mathbf{k}[z]$-modules (these ranks are equal). The shift of grading gives an equivalence of dg-categories

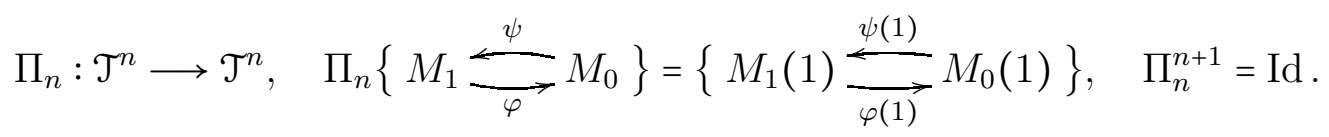


The following is an adaptation of the main result of [Tak05]. We omit the proof as well as the proofs of the next few followup statements, as they are similar to loc. cit.

Theorem 2.3.6. (a) The triangulated category $H^{0} \mathcal{T}^{n}$ is equivalent to $D^{(2)}\left(A_{n}\right.$-mod).

(b) Under this equivalence, the functor induced by $\Pi_{n}$, corresponds to the derived Coxeter functor $C_{n}$.

The rank one objects of $\mathcal{T}^{n}$ have the form

$$
\begin{gathered}
{[i, j]=\left\{R(i) \underset{z^{i-j}}{\stackrel{z^{j-i}}{\rightleftarrows}} R(j)\right\}, \quad i \neq j,} \\
{[i, i]=\left\{R(i) \underset{z^{n+1}}{\stackrel{\mathrm{Id}}{\rightleftarrows}} R(i)\right\}, \quad[i, i]^{\prime}=\left\{R(i) \underset{\mathrm{Id}}{\stackrel{z^{n+1}}{\rightleftarrows}} R(i)\right\}, \quad i \in \mathbb{Z} /(n+1),}
\end{gathered}
$$

where the exponents are to be interpreted via the identification $\mathbb{Z} /(n+1) \cong\{0,1, \ldots, n\}$. It is clear that

$$
\Sigma[i, j]=[j, i], \quad i \neq j, \quad \Sigma[i, i]=[i, i]^{\prime} .
$$

One verifies by computing Hom-complexes that

$$
[i, i] \simeq\left[i, i^{\prime}\right] \simeq 0 \in H^{0} \mathcal{T}^{n}
$$

are zero objects in $H^{0} \mathcal{T}^{n}$.

Proposition 2.3.8. The $[i, j]$ exhaust all isomorphism classes of indecomposable objects in $H^{0} \mathcal{T}^{n}$

Proposition 2.3.9. Let $i, j, k \in \mathbb{Z} /(n+1)$ be distinct elements, in counterclockwise cyclic order. Then the degree 0 morphisms

$$
\begin{gathered}
\alpha_{i j k}=\left(\begin{array}{cc}
1 & 0 \\
0 & z^{k-j}
\end{array}\right) \in \operatorname{Hom}^{0}([i, j],[i, k]), \quad \beta_{i j k}=\left(\begin{array}{cc}
z^{j-i} & 0 \\
0 & 1
\end{array}\right) \in \operatorname{Hom}^{0}([i, k],[j, k]), \\
\gamma_{i j k}=\left(\begin{array}{cc}
0 & z^{i-k} \\
1 & 0
\end{array}\right) \in \operatorname{Hom}^{0}([j, k],[k, i]),
\end{gathered}
$$

are closed and induce a distinguished triangle in $H^{0} \mathcal{T}^{n}$ :

$$
[i, j] \stackrel{\alpha_{i j k}}{\longrightarrow}[i, k] \stackrel{\beta_{i j k}}{\longrightarrow}[j, k] \stackrel{\gamma_{i j k}}{\longrightarrow}[k, i]=\Sigma[i, k] .
$$

The equivalence of Theorem 2.3.6 can be chosen so that

$$
\mathbf{e}_{i j} \mapsto[i, j], \quad i \neq j,
$$

and the triangles of Example 2.3.4 correspond to those of Proposition 2.3.9. For this, note that each of the following two diagrams

$$
\begin{gathered}
\mathcal{R}_{n}^{\triangleleft}=\left\{[0,1] \stackrel{\alpha_{012}}{\longrightarrow}[0,2] \stackrel{\alpha_{023}}{\longrightarrow} \cdots \stackrel{\alpha_{0, n-1, n}}{\longrightarrow}[0, n]\right\}, \\
\mathcal{R}_{n}^{\triangleright}=\left\{[0, n] \stackrel{\beta_{01 n}}{\longrightarrow}[1, n] \stackrel{\beta_{12 n}}{\longrightarrow} \cdots \stackrel{\beta_{n-2, n-1, n}}{\longrightarrow}[n-1, n]\right\},
\end{gathered}
$$


can be considered as a representation of $A_{n}$ in $\mathcal{T}^{n}$. The dg-functor

$$
\Phi_{n}^{\triangleleft}: C^{(2)}\left(A_{n}-\bmod \right) \longrightarrow \mathcal{T}^{n}, \quad V^{\bullet} \mapsto \operatorname{RHom}_{A_{n}}\left(\mathcal{R}_{n}^{\triangleleft}, V^{\bullet}\right)
$$

establishes an equivalence satisfying (2.3.10). A different equivalence $\Phi_{n}^{\triangleright}$ can be constructed using $\mathcal{R}_{n}^{\triangleright}$.

\subsection{The dg-categories $\varepsilon^{n}$ and their cocyclic structure}

We denote by $\mathcal{E}^{n}=\operatorname{LF}\left(Q^{n}\right)$ the dg-category of loop factorizations of the $\mathbb{Z}_{+}$-category $\mathbb{Q}^{n}$ from Example 2.1.3. Because the construction $\mathcal{C} \mapsto L F(\mathcal{C})$ is covariantly functorial in the $\mathbb{Z}_{+}$-category $\mathcal{C}$, we obtain immediately:

Proposition 2.4.1. The collection $\mathcal{E}^{\bullet}=\left(\mathcal{E}^{n}\right)_{n \geq 0}$, forms a cocyclic object in dgcat $^{(2)}$.

For $i, j \in \mathbb{Z} /(n+1), i \neq j$, let $\varphi_{i j}$ be the shortest oriented path from $i$ to $j$ in the circular quiver $Q^{n}$. Recall that $w_{i}$ denotes the full circle path beginning and ending at $i$. The objects of $\mathcal{E}^{n}$ are exhausted by the following:

$$
\begin{gathered}
E_{i j}=\left\{i \stackrel{\varphi_{\varphi_{i j}}}{\rightleftharpoons}\right\}, \quad i \neq j, \\
E_{i i}=\left\{i \underset{w_{i}}{\stackrel{\mathrm{Id}}{\leftrightarrows}} i\right\}, \quad E_{i i}^{\prime}=\left\{i \stackrel{w_{i}}{\rightleftharpoons} i\right\}, \quad i \in \mathbb{Z} /(n+1) .
\end{gathered}
$$

Proposition 2.4.3. The correspondence

$$
E_{i j} \mapsto[i, j], \quad E_{i i}^{\prime} \mapsto[i, i]^{\prime}
$$

extends to a fully faithful dg-functor $\epsilon_{n}: \mathcal{E}^{n} \rightarrow \mathcal{T}^{n}$.

Proof. The definitions imply at once the identifications of the Hom-complexes.

Let $D_{\text {ind }}^{(2)}\left(A_{n}\right.$-mod $)$ be the full subcategory in $D^{(2)}\left(A_{n}\right.$-mod $)$ formed by all indecomposable objects, including the zero object. The above proposition, together with Theorem 2.3.6(a), implies an equivalence of categories

$$
H^{0}\left(\mathcal{E}^{n}\right) \simeq D_{\text {ind }}^{(2)}\left(A_{n}-\bmod \right)
$$

Let $\mathcal{A}^{n}$ be the $\mathbf{k}$-linear envelope of the quiver $A_{n}$, considered as a differential $\mathbb{Z} / 2$ graded category concentrated in even degree with zero differential. The diagrams (2.3.11) can be considered as dg-functors

$$
r_{n}^{\triangleleft}, r_{n}^{\triangleright}: \mathcal{A}^{n} \longrightarrow \mathcal{E}^{n}
$$

Proposition 2.4.6. The $d g$ functors $r_{n}^{\triangleleft}, r_{n}^{\triangleright}, \epsilon_{n}$ are Morita equivalences. 
Proof. For $\epsilon_{n}$, which is an embedding of a full dg-category, the statement follows from Proposition 2.3.8 each object of $H^{0} \mathcal{T}^{n}$ is isomorphic to a direct sum of objects from $\operatorname{Im}\left(\epsilon_{n}\right)$. To prove the statement for $r_{n}^{\triangleleft}$, we note that is is quasi-fully faithful: induces quasi-isomorphisms on Hom-complexes. By the above, it is enough to prove that $\epsilon_{n} \circ r_{n}^{\triangleleft}$ is a Morita equivalence. This follows because, by Proposition 2.3.9, each object of $H^{0} \epsilon_{n}\left(\mathcal{E}^{n}\right)$ and therefore, by the above, each object of $H^{0} \mathcal{T}^{n}$, is obtained from objects $\operatorname{in} \operatorname{Im}\left(\epsilon_{n} \circ r_{n}^{\triangleleft}\right)$ by taking iterated cones of morphisms. The case of $r_{n}^{\triangleright}$ is similar.

\subsection{1 $\mathcal{E}^{2}$ and distinguished triangles}

In Section 5 below, we provide a thorough analysis of the universal property of the cyclic object $\mathcal{E} \bullet$ in terms of Waldhausen's S-construction. Here, we use a more explicit approach identifying the dg category $\mathcal{E}^{2}$ as a universal distinguished triangle category.

Let $\mathcal{T}$ be a triangulated category equipped with a 2-periodic dg enhancement. Due to the 2-periodicity, a triangle

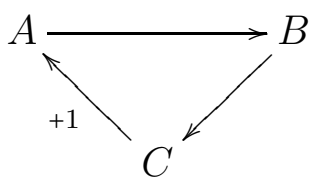

in $\mathcal{T}$ can be depicted more symmetrically via the diagram

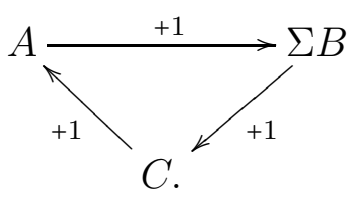

We will refer to the latter diagram as a symmetric triangle in $\mathcal{T}$. A symmetric triangle in $\mathcal{T}$ is called distinguished if its corresponding asymmetric triangle is distinguished. We denote by $\mathscr{F}$ the 2 -periodic dg category with three objects 0,1 , and 2 , freely generated by closed morphisms $p_{10}: 0 \rightarrow 1, p_{21}: 1 \rightarrow 2$, and $p_{02}: 2 \rightarrow 0$ of degree 1 . Given 2-periodic dg categories $\mathcal{A}, \mathcal{B}$, we denote by $[\mathcal{A}, \mathcal{B}]$ the set of morphisms from $\mathcal{A}$ to $\mathcal{B}$ in the homotopy category $\mathrm{Ho}\left(\right.$ dgcat $\left.^{(2)}\right)$.

Proposition 2.4.7. Let $\mathcal{T}$ be a triangulated category with 2-periodic dg enhancement $\mathcal{A}$. Then the set $[\mathscr{F}, \mathcal{A}]$ of morphisms in $\mathrm{Ho}\left(\mathrm{dgcat}^{(2)}\right)$ is in canonical bijection with the set of isomorphism classes of triangles in $\mathcal{T}$.

Proof. The dg category $\mathscr{F}$ is obtained by iterated pushouts along generating cofibrations of dgcat ${ }^{(2)}$ and hence cofibrant. By the usual model category formalism, we can therefore compute the set $\operatorname{Hom}_{\mathrm{Ho}\left(\operatorname{dgcat}^{(2)}\right)}(\mathscr{F}, \mathcal{A})$ as homotopy classes of maps from $\mathscr{F} \rightarrow \mathcal{A}$. Here, two dg functors $f: \mathscr{F} \rightarrow \mathcal{A}$ and $g: \mathscr{F} \rightarrow \mathcal{A}$ are homotopic, if there exists a commutative 
diagram

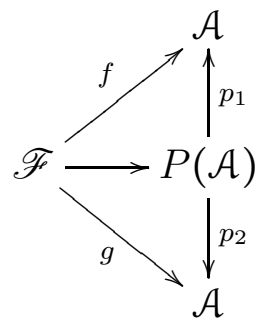

in dgcat $^{(2)}$. Here, $P(\mathcal{A})$ denotes a path object for $\operatorname{dgcat}^{(2)}$ which can be explicitly constructed as follows (cf. [Tab07]). The objects of $P(\mathcal{A})$ are pairs $(x, y)$ of objects of $\mathcal{A}$ equipped with a closed morphism $f: x \rightarrow y$ of degree 0 which becomes an isomorphism in $H^{0}(\mathcal{A})$. The mapping complex between objects $(x, y, f)$ and $\left(x^{\prime}, y^{\prime}, f^{\prime}\right)$ is defined as the suspension of the cone of the map

$$
\operatorname{Hom}_{\mathcal{A}}\left(x, x^{\prime}\right) \oplus \operatorname{Hom}_{\mathcal{A}}\left(y, y^{\prime}\right) \stackrel{\left(f_{*}^{\prime},-f^{*}\right)}{\longrightarrow} \operatorname{Hom}_{\mathcal{A}}\left(x, y^{\prime}\right) .
$$

Now consider the obvious map of sets

$$
\operatorname{Hom}_{\text {dgcat }^{(2)}}(\mathscr{F}, \mathcal{A}) \longrightarrow\left\{\text { symmetric triangles in } \mathcal{T}=H^{0}(\mathcal{A})\right\}
$$

which is easily seen to be surjective. Unravelling the definition of the path $\mathrm{dg}$ category, it is straightforward to verify that two $\operatorname{dg}$ functors from $\mathscr{F}$ to $\mathcal{A}$ are homotopic if and only if the corresponding symmetric triangles are isomorphic as diagrams in $\mathcal{T}$.

Remark 2.4.8. Given 2-periodic dg categories $\mathcal{A}, \mathcal{B}$, the set of maps $[\mathcal{A}, \mathcal{B}]$ can be canonically identified with the set of connected components of the mapping $\operatorname{space} \operatorname{Map}(\mathcal{A}, \mathcal{B})$ of the simplicial localization of dgcat ${ }^{(2)}$ along quasi-equivalences. Therefore, Proposition 2.4.7 provides an interpretation of $\operatorname{Map}(\mathscr{F}, \mathcal{A})$ as a classifying space for triangles in $\mathcal{T}$.

By Proposition 2.3.9, the diagram in $H^{0}\left(\mathcal{E}^{2}\right)$

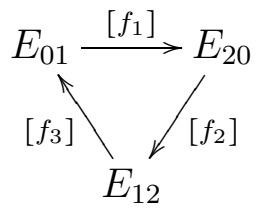

with

$$
f_{1}=\left(\begin{array}{cc}
0 & z \\
1 & 0
\end{array}\right) \quad f_{2}=\left(\begin{array}{cc}
0 & z^{2} \\
1 & 0
\end{array}\right) \quad f_{3}=\left(\begin{array}{cc}
0 & z \\
1 & 0
\end{array}\right)
$$

is a symmetric distinguished triangle. Analyzing the null homotopies in $\mathcal{E}^{2}$ of the pairwise composites of the morphisms $f_{i}$, we can lift (2.4.9) to a diagram in the $\mathrm{dg}$ category $\mathcal{E}^{2}$

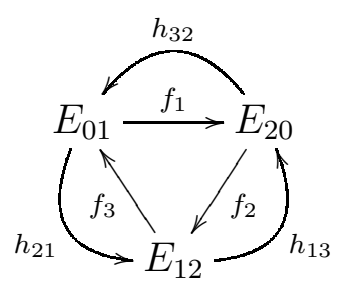


with

$$
h_{21}=\left(\begin{array}{ll}
0 & 0 \\
1 & 0
\end{array}\right) \quad h_{32}=\left(\begin{array}{ll}
0 & 0 \\
1 & 0
\end{array}\right) \quad h_{13}=\left(\begin{array}{ll}
0 & 0 \\
1 & 0
\end{array}\right)
$$

satisfying

$$
d\left(h_{i j}\right)=f_{i} f_{j}
$$

Further, we observe that there are relations in $\mathcal{E}^{2}$

$$
h_{32} f_{1}+f_{3} h_{21}=\operatorname{id}_{E_{01}} \quad h_{13} f_{2}+f_{1} h_{32}=\operatorname{id}_{E_{20}} \quad h_{21} f_{3}+f_{2} h_{13}=\operatorname{id}_{E_{12}} .
$$

Equivalently, we can reformulate our observation by saying that there exists a dg functor $f: \mathcal{D} \rightarrow \mathcal{E}^{2}$ where $\mathcal{D}$ is the 2-periodic dg category with objects $0,1,2$, generated by closed morphisms $f_{1}: 0 \rightarrow 1, f_{2}: 1 \rightarrow 2, f_{3}: 2 \rightarrow 0$ of degree 1 , and morphisms $h_{21}: 0 \rightarrow 2$, $h_{32}: 1 \rightarrow 0, h_{13}: 2 \rightarrow 1$ of degree 1 satisfying (2.4.10) and (2.4.11).

Proposition 2.4.12. The functor $f: \mathcal{D} \rightarrow \mathcal{E}^{2}$ is a Morita equivalence.

Proof. Left to the reader.

We will now give an interpretation of the set of morphisms $\left[\mathcal{E}^{2}, \mathcal{A}\right]$ in $\operatorname{Ho}\left(\operatorname{dgcat}^{(2)}\right)$ in terms of distinguished triangles in $H^{0}(\mathcal{A})$. By Proposition 2.4.12, this set is in natural bijection with the set of morphisms $[\mathcal{D}, \mathcal{A}]$. To get a more explicit hold on this morphism set, we may utilize the model structure on dgcat $^{(2)}$ and pass to a cofibrant replacement $p: \widetilde{\mathcal{D}} \rightarrow \mathcal{D}$.

The dg category $\widetilde{\mathcal{D}}$ can be explicitly described as follows. Denote by $\mathbb{Q}^{2}$ the cyclic quiver with vertices 0,1 , and 2 , and arrows $0 \rightarrow 1,1 \rightarrow 2$, and $2 \rightarrow 0$. Let $\widetilde{\mathcal{D}}$ denote the 2-periodic dg category with objects 0,1 , and 2 , obtained by adjoining, for every path $\gamma$ in $Q^{2}$ a (not necessarily closed) morphism $p_{\gamma}: s(\gamma) \rightarrow t(\gamma)$ of degree 1 , with differential given by

$$
d\left(p_{\gamma}\right)= \begin{cases}\sum_{\beta \circ \alpha=\gamma} p_{\beta} p_{\alpha}-\mathrm{id}_{i} & \text { if } \gamma \text { is a degree } 1 \text { cycle centered at } i \\ 0 & \text { if } \gamma \text { has length } 1, \\ \sum_{\beta \circ \alpha=\gamma} p_{\beta} p_{\alpha} & \text { for all other cases. }\end{cases}
$$

Since the category $\mathscr{F}$ is freely generated by paths in $\mathcal{Q}^{2}$ of length 1 , we have a natural embedding $i: \mathscr{F} \rightarrow \widetilde{\mathcal{D}}$. The functor $i$ is a cofibration, since it can be obtained by iterated pushouts along generating cofibrations. In particular, the $\operatorname{dg}$ category $\widetilde{\mathcal{D}}$ is cofibrant. The functor $p: \widetilde{\mathcal{D}} \rightarrow \mathcal{D}$ is simply obtained by sending all morphisms corresponding to paths of length $\geq 3$ to 0 .

Proposition 2.4.13. The functor $p: \widetilde{\mathcal{D}} \rightarrow \mathcal{D}$ is a quasi-equivalence.

Proof. Left to the reader. 
In summary, we have a dg functors

$$
\mathscr{F} \stackrel{i}{\longrightarrow} \widetilde{\mathcal{D}} \stackrel{p}{\longrightarrow} \mathcal{D} \stackrel{f}{\longrightarrow} \mathcal{E}^{2}
$$

with $\mathscr{F}$ and $\widetilde{\mathcal{D}}$ cofibrant, $p$ a quasi-equivalence, and $f$ a Morita equivalence.

Proposition 2.4.14. Let $\mathcal{T}$ be a triangulated category with 2-periodic dg enhancement $\mathcal{A}$.

(1) $A$ dg functor $\mathscr{F} \rightarrow \mathcal{A}$ representing a triangle in $\mathcal{T}$ lifts to a dg functor $\widetilde{\mathcal{D}} \rightarrow \mathcal{A}$ if and only if the triangle is distinguished.

(2) The pullback map

$$
[\widetilde{\mathcal{D}}, \mathcal{A}] \longrightarrow[\mathscr{F}, \mathcal{A}]
$$

is an inclusion with image given by the subset of isomorphism classes of distinguished symmetric triangles in $\mathcal{T}$.

Proof. By definition, a distinguished symmetric triangle in $\mathcal{T}$ is a symmetric triangle in $\mathcal{T}$ that is isomorphic as a diagram in $\mathcal{T}$ to a cone triangle of the form

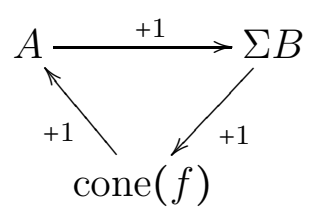

where $f: A \rightarrow B$ is a closed degree 0 morphism in $\mathcal{A}$ and cone $(f)$ denotes the mapping cone construction in $\mathcal{A}$ (which is pre-triangulated in the sense of [BK91]). Therefore, by the argument of Proposition 2.4.7, a symmetric triangle, represented by a dg functor $F: \mathscr{F} \rightarrow \mathcal{A}$, is distinguished, if and only if there exists a commutative diagram

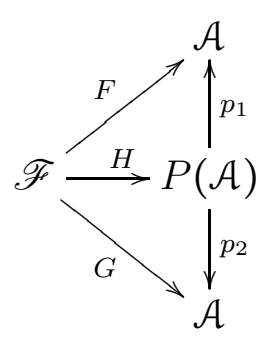

in dgcat ${ }^{(2)}$ where the symmetric triangle represented by $G$ is a cone triangle. We explicitly verify that any cone triangle in $\mathcal{T}$ can be lifted to a $\operatorname{dg}$ functor $\widetilde{\mathcal{D}} \rightarrow \mathcal{A}$ as follows. By construction of the object cone $(f)$ as a twisted complex, it comes equipped with morphisms in $\mathcal{A}$ as depicted in the diagram

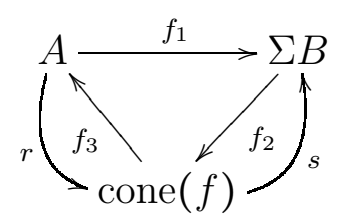


with $f_{1}, f_{2}, f_{3}$ closed morphisms of degree 1 , satisfying $f_{2} f_{1}=d r, f_{1} f_{3}=d s, f_{3} f_{2}=0$, $r f_{3}+f_{2} s=\mathrm{id}_{\text {cone }(f)}, f_{3} r=\mathrm{id}_{A}, s f_{2}=\mathrm{id}_{\Sigma B}$. Comparing these formulas to the defining formulas (2.4.10) and (2.4.11) of $\mathcal{D}$, there is an apparent $\operatorname{dg}$ functor $\mathcal{D} \rightarrow \mathcal{A}$ representing this triangle which can be precomposed with $p: \widetilde{\mathcal{D}} \rightarrow \mathcal{D}$ to obtain a dg functor $\widetilde{\mathcal{D}} \rightarrow \mathcal{A}$.

Hence, we obtain a commutative diagram

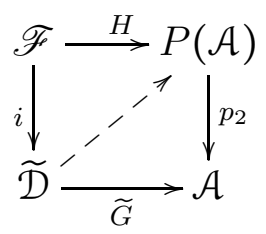

in dgcat $^{(2)}$. Since $i$ is a cofibration and $p_{2}$ a trivial fibration, we can fill in the indicated functor $\widetilde{\mathcal{D}} \rightarrow P(\mathcal{A})$. Composing this functor with $p_{1}$, we obtain a functor $\widetilde{F}: \widetilde{\mathcal{D}} \rightarrow \mathcal{A}$ lifting $F$. On the other hand, an easy calculation shows that any functor $\mathscr{F} \rightarrow \mathcal{A}$ which is a restriction of a functor $\widetilde{\mathcal{D}} \rightarrow \mathcal{A}$ represents a distinguished symmetric triangle. We have shown that the map $[\widetilde{\mathcal{D}}, \mathcal{A}] \rightarrow[\mathscr{F}, \mathcal{A}]$ surjects onto those functors which represent distinguished triangles in $\mathcal{T}$. It remains to show that the map is injective. We have a commutative diagram in dgcat $^{(2)}$

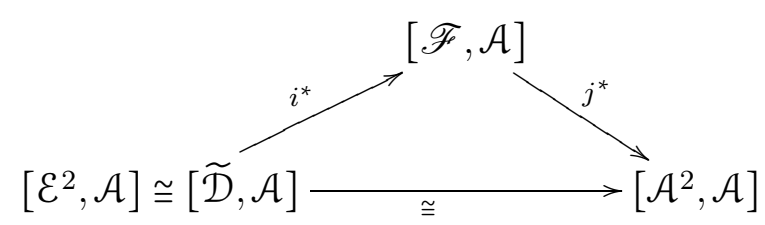

where the horizontal map is a bijection by Proposition 2.4.6. In particular, $i^{*}$ must be injective which concludes the argument.

Remark 2.4.15. In analogy with Remark 2.4.8, Proposition 2.4.14 implies that the connected components of the mapping $\operatorname{space} \operatorname{Map}\left(\mathcal{E}^{2}, \mathcal{A}\right) \simeq \operatorname{Map}(\mathcal{D}, \mathcal{A}) \simeq \operatorname{Map}(\widetilde{\mathcal{D}}, \mathcal{A})$ are in bijective correspondence with isomorphism classes of distinguished triangles in $\mathcal{T}$.

Remark 2.4.16. The analogue of statement (1) in Proposition 2.4.14 for $A_{\infty}$-categories is a result due to M. Kontsevich (cf. Proposition 3.8 in [Sei08]). While in the context of dg-categories we are forced to work with the (rather large) cofibrant replacement $\widetilde{\mathcal{D}}$ of $\mathcal{D}$, using $A_{\infty}$-categories and $A_{\infty}$-functors, one does not have to replace $\mathcal{D}$. On the contrary, one can pass to an even more economic minimal model of $\mathcal{D}$ : a simple homological perturbation calculation shows that a minimal model $\overline{\mathcal{D}}$ is given by the $\mathcal{A}_{\infty}$-category with objects $0,1,2$, obtained by adjoining closed morphisms $f_{1}: 0 \rightarrow 1, f_{2}: 1 \rightarrow 2, f_{3}: 2 \rightarrow 1$ of degree 1 whose pairwise composition equals 0 , equipped with triple operations

$$
m_{3}\left(f_{3}, f_{2}, f_{1}\right)=\mathrm{id}_{0} \quad m_{3}\left(f_{1}, f_{3}, f_{2}\right)=\mathrm{id}_{1} \quad m_{3}\left(f_{2}, f_{1}, f_{3}\right)=\mathrm{id}_{2} .
$$

The natural morphism $\mathscr{F} \rightarrow \overline{\mathcal{D}}$ has the property that, given a 2-periodic $A_{\infty}$-category $\mathcal{A}$, a (symmetric) triangle in $H^{0}(\mathcal{A})$ represented by an $A_{\infty}$-functor $\mathscr{F} \rightarrow \mathcal{A}$, is exact if and 
only if this functor admits a lift to an $A_{\infty}$-functor $\overline{\mathcal{D}} \rightarrow \mathcal{A}$. Of course, the simplicity of the category $\overline{\mathcal{D}}$, when compared to $\widetilde{\mathcal{D}}$, comes at a price: the complexity is now hidden in the amount of data needed to specify an $A_{\infty}$-functor.

\subsection{Cyclic duality and Morita duality}

Given a $\mathbb{Z}_{+}$-category $\mathcal{C}$, the dual $\mathbb{Z}_{+}$-category is defined as $\mathcal{C}^{\vee}:=\underline{\mathrm{Fun}}_{\mathbb{Z}_{+}}\left(\mathcal{C}, Q^{0}\right)$, see [Dr04]. The duality operation provides a functor

$$
(-)^{\vee}: \mathbb{Z}_{+}-\mathrm{C} a t^{\mathrm{op}} \longrightarrow \mathbb{Z}_{+}-\mathrm{C} a t .
$$

It is not a perfect duality, i.e., the canonical functor $\mathcal{C} \rightarrow \mathrm{C}^{\vee \vee}$ need not be an isomorphism, nor an equivalence of $\mathbb{Z}_{+}$-categories. However, it induces a perfect duality functor on cyclic ordinals

$$
(-)^{*}: \Lambda^{\mathrm{op}} \longrightarrow \Lambda, \quad I \mapsto I^{*}, \quad I^{* *}=I, \quad Q^{I^{*}} \simeq\left(Q^{I}\right)^{\vee} .
$$

Explicitly, for a cyclic ordinal $I$, the dual cyclic ordinal $I^{*}$ is the set of interstices, i.e., of minimal cyclic intervals in $I$ :

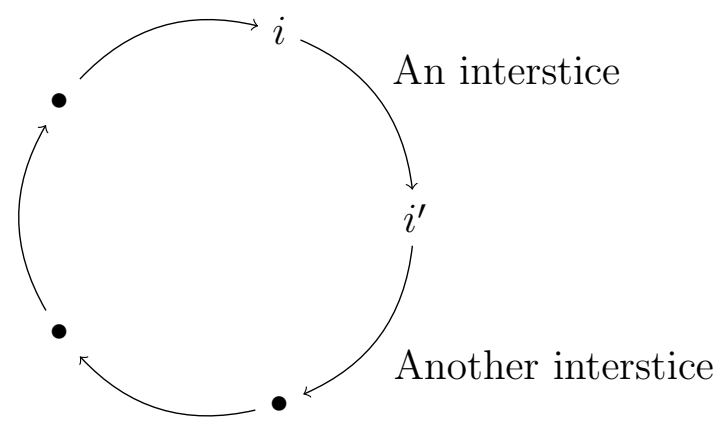

On the other hand, as we have seen in $\$ 1.1$, the Morita homotopy category $\operatorname{Ho}\left(\operatorname{dgcat}^{(2)}\right)$ admits a duality functor

$$
(-)^{\vee}: \operatorname{Ho}\left(\operatorname{dgcat}^{(2)}\right)^{\text {op }} \longrightarrow \operatorname{Ho}\left(\operatorname{dgcat}^{(2)}\right), \quad \mathcal{A} \mapsto \mathcal{A}^{\vee}=R \underline{\operatorname{Hom}}\left(\mathcal{A}, \operatorname{Perf}_{\mathbf{k}}^{(2)}\right) .
$$

The goal of this section is to understand how the functor

$$
\mathrm{MF}: \mathbb{Z}_{+}-\mathrm{Cat} \longrightarrow \operatorname{dgcat}^{(2)}
$$

relates these duality functors.

To this end, for a $\mathbb{Z}_{+}$-category $\mathcal{C}$ we define a dg-category $\widetilde{\mathrm{LF}}(\mathcal{C})$ completely analogous to $\operatorname{LF}(\mathcal{C})$ but with the substitutions $(\varphi, \psi) \mapsto(\psi,-\varphi)$ and $\left(\varphi^{\prime}, \psi^{\prime}\right) \mapsto\left(\psi^{\prime},-\varphi^{\prime}\right)$ in the formulas (2.2.3) for the differentials on the mapping complexes. To signify this sign change in a suggestive way, we denote the objects of $\widetilde{\mathrm{LF}}(\mathcal{C})$ by

$$
x_{1} \stackrel{-\varphi}{\longleftarrow} x_{0}
$$


Remark 2.5.1. Assume that $\mathcal{C}$ itself has a "duality" functor which a $\mathbb{Z}_{+}$-equivalence $\mathrm{C}^{\mathrm{op}} \longrightarrow \mathrm{C}^{\vee}, x \mapsto x^{\vee}$. Then dualizing an object

$$
x_{1} \underset{\varphi}{\stackrel{\psi}{\longrightarrow}} x_{0}
$$

of $\operatorname{LF}(\mathcal{C})$ using the Koszul signs rule yields the object

$$
x_{1}^{\vee} \underset{\psi^{\vee}}{\stackrel{-\varphi^{\vee}}{\longrightarrow}} x_{0}^{\vee}
$$

of $\widetilde{\mathrm{LF}}\left(\mathrm{C}^{\vee}\right)$. In such a case, this association extends to an isomorphism of dg-categories $\mathrm{LF}(\mathcal{C})^{\mathrm{op}} \cong \widetilde{\mathrm{LF}}\left(\mathrm{C}^{\vee}\right)$.

Given a $\mathbb{Z}_{+}$-category $\mathcal{C}$, there is a natural functor of categories

$$
\mathrm{C}^{\vee} \times \mathrm{C} \longrightarrow \operatorname{Vect}_{\mathbf{k}},(F, x) \mapsto F \otimes x
$$

where we define $F \otimes x$ to be the free $\mathbf{k}$-vector space on the set $\operatorname{Hom}_{\mathbb{Q}^{0}}(0, F(x))$.

Definition 2.5.3. Let $\mathcal{C}$ be a $\mathbb{Z}_{+}$-category. We define a dg functor

$$
\eta_{\mathcal{e}}: \widetilde{\mathrm{LF}}\left(\mathrm{C}^{\vee}\right) \otimes \operatorname{LF}(\mathcal{C}) \rightarrow \operatorname{Mod}_{\mathbf{k}}^{(2)}
$$

by the formula

$$
\left(F_{1} \underset{\xi}{\stackrel{-\theta}{\rightleftarrows}} F_{0}, x_{1} \underset{\varphi}{\stackrel{\psi}{\rightleftarrows}} x_{0}\right) \mapsto\left(F_{1} \underset{\xi}{\stackrel{-\theta}{\rightleftarrows}} F_{0}\right) \otimes\left(x_{1} \underset{\varphi}{\stackrel{\psi}{\rightleftarrows}} x_{0}\right)
$$

where the tensor product is given by (2.5.2) and differential determined by the Koszul rule where $F_{0}$ and $x_{0}$ are considered of even degree while $F_{1}$ and $x_{1}$ are considered of odd degree.

Proposition 2.5.4. For any $\mathbb{Z}_{+}$-functor $F: \mathcal{C} \rightarrow \mathcal{D}$ of $\mathbb{Z}_{+}$-categories, the diagram of $d g$ categories

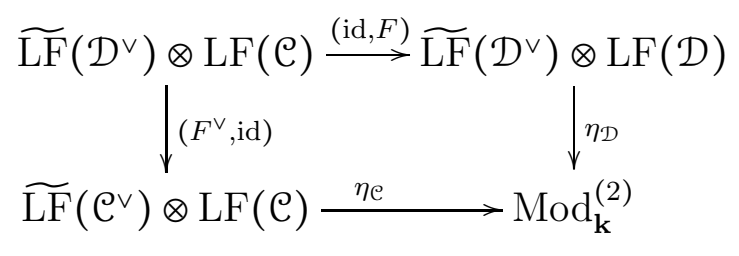

commutes.

Proof. This follows immediately from the fact that the diagram of categories

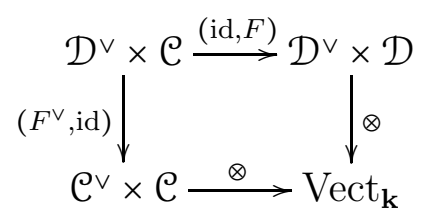

commutes. 
Proposition 2.5.5. Let $\mathrm{C}=\mathrm{Q}^{n}$ Then the Yoneda embedding

$$
\mathfrak{e}^{\mathrm{op}} \longrightarrow \underline{\mathrm{Fun}}_{\mathbb{Z}_{+}}\left(\mathcal{C}, \mathbb{Z}_{+}-\mathcal{S e t}\right)
$$

factors over the canonical embedding $\mathrm{C}^{\vee}=\underline{\mathrm{Fun}}_{\mathbb{Z}_{+}}(\mathcal{C},\langle 0\rangle) \rightarrow \underline{\mathrm{Fun}}_{\mathbb{Z}_{+}}\left(\mathcal{C}, \mathbb{Z}_{+}-\mathcal{S}\right.$ et $)$ and induces $a \mathbb{Z}_{+}$-equivalence between $\mathrm{C}^{\mathrm{op}}$ and the dual $\mathbb{Z}_{+}$-category $\mathrm{C}^{\vee}$, and hence, by Remark 2.5.1, an isomorphism of dg-categories

$$
\mathrm{LF}(\mathcal{C})^{\mathrm{op}} \cong \widetilde{\mathrm{LF}}\left(\mathrm{C}^{\vee}\right)
$$

Proposition 2.5.6. Let $\mathcal{C}=Q^{n}$. Then there is a commutative diagram

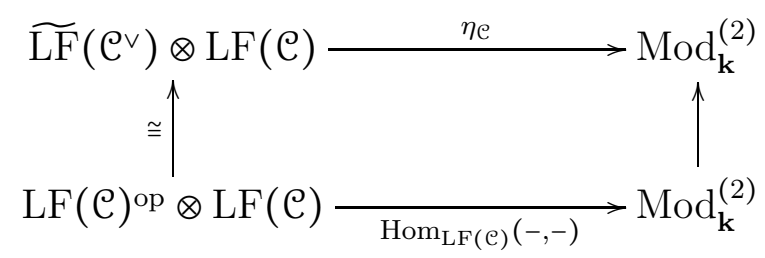

In particular, the functor

$$
\widetilde{\mathrm{LF}}\left(\mathrm{C}^{\vee}\right) \longrightarrow \operatorname{Fun}\left(\operatorname{LF}(\mathcal{C}), \operatorname{Mod}_{\mathbf{k}}^{(2)}\right)
$$

adjoint to $\eta_{\mathcal{e}}$, factors over $R \underline{\operatorname{Hom}}\left(\operatorname{LF}(\mathcal{C}), \operatorname{Perf}_{\mathbf{k}}\right) \subset \operatorname{Fun}\left(\operatorname{LF}(\mathcal{C}), \operatorname{Mod}_{\mathbf{k}}^{(2)}\right)$. The induced functor

$$
\mathrm{LF}\left(\mathrm{e}^{\vee}\right) \longrightarrow R \underline{\operatorname{Hom}}\left(\mathrm{LF}(\mathrm{C}), \operatorname{Mod}_{\mathbf{k}}^{(2)}\right)
$$

is a Morita equivalence which exhibits the dg-category $\widetilde{\mathrm{LF}}\left(\mathrm{C}^{\vee}\right)$ as the Morita dual of $\mathrm{LF}(\mathrm{C})$.

Corollary 2.5.7. We have a commutative diagram

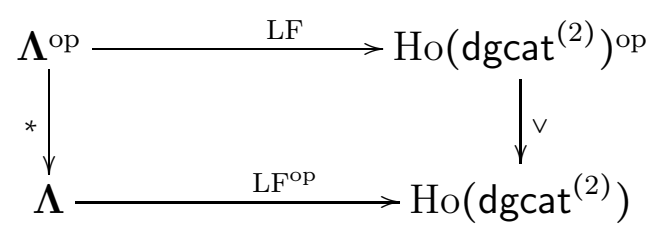

relating the duality functors on $\boldsymbol{\Lambda}$ and $\mathrm{Ho}\left(\operatorname{dgcat}^{(2)}\right)$.

\section{Cyclic 2-Segal objects}

\subsection{The 1- and 2-Segal conditions}

We denote by $\Delta$ the category of finite nonempty ordinals $[n]=\{0,1, \ldots, n\}$ and monotone maps. For a category $\mathbf{C}$, a simplicial object $X$ in $\mathbf{C}$ is defined to be a functor $X: \Delta^{\mathrm{op}} \rightarrow \mathbf{C}$ and we denote by $\mathbf{C}_{\Delta}$ the category of simplicial objects in $\mathbf{C}$. Similarly, a cyclic object 
$X$ in $\mathbf{C}$ is defined to be a functor $X: \Lambda \rightarrow \mathbf{C}$, where $\Lambda$ denotes Connes' cyclic category. We denote by $\mathbf{C}_{\Lambda}$ the category of cyclic objects in $\mathbf{C}$. There is an embedding

$$
\varsigma: \Delta \longrightarrow \Lambda
$$

which associates to a finite ordinal the cyclic ordinal corresponding to it by cyclic closure. That is, $\varsigma([n])=\langle n\rangle$. Thus a cyclic object $X$ in $\mathbf{C}$ gives rise to a simplicial object $\varsigma^{*} X$ (often also denoted $X$ ) together with endomorphisms (cyclic rotations) $t_{n}: X_{n} \rightarrow X_{n}$, satisfying the well known identities [Con94, Ch. III, App. A:

$$
\begin{gathered}
t_{n}^{n+1}=\mathrm{Id}, \\
\partial_{i} t_{n}=t_{n-1} \partial_{i-1}, \quad \text { for } 1 \leq i \leq n, \quad \text { while } \quad \partial_{0} t_{n}=\partial_{n} \\
s_{i} t_{n}=t_{n+1} s_{i-1} \quad \text { for } 1 \leq i \leq n, \quad \text { while } \quad s_{0} t_{n}=t_{n+1}^{2} s_{n} .
\end{gathered}
$$

Suppose now that $\mathbf{C}$ has limits. For a simplicial set $K$ and a simplicial object $X \in \mathbf{C}_{\Delta}$ we denote, following [HSS1, the space of $K$-membranes in $X$ as the object

$$
(K, X)=\lim _{\left\{\Delta^{p} \rightarrow K\right\}}^{\mathbf{C}} X_{p} \in \mathbf{C} .
$$

Here the limit is taken over all simplices of $K$. The functor

$$
\Upsilon_{*} X: \mathcal{S e t}_{\Delta}^{\mathrm{op}} \longrightarrow \mathbf{C}, \quad K \mapsto(K, X),
$$

is the right Kan extension of $X: \Delta^{\mathrm{op}} \rightarrow \mathrm{C}$ along the Yoneda embedding $\Upsilon: \Delta^{\mathrm{op}} \rightarrow \mathcal{S} e t_{\Delta}^{\mathrm{op}}$.

Suppose now that $\mathbf{C}$ carries a model structure. Then we can define derived functors of the projective limit of $\mathbf{C}$-valued diagrams [DHKS04], and will refer to them as homotopy limit functors. We will use the notation holim $\longleftarrow_{a \in A}^{\mathbf{C}} Z_{a}$ for the homotopy limit of a diagram $\left(Z_{a}\right)_{a \in A}$. Similarly for the derived functors of Yoneda extension functors, see loc. cit. In particular, we define the derived space of $K$-membranes in $X$, denote $(K, X)_{R}$ as

$$
(K, X)_{R} \simeq \operatorname{holim}_{\left\{\Delta^{p} \rightarrow K\right\}}^{\mathbf{C}} X_{p} \in \mathbf{C} .
$$

See [HSS1, §5.1] for more details. We will need two particular examples of simplicial sets.

Examples 3.1.3. (a) We denote by $I[n] \subset \Delta^{n}$ be the simplicial set ("subdivided interval") corresponding to the oriented graph

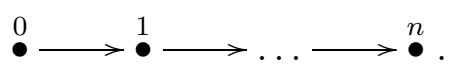

(b) Let $P_{n+1}$ be the standard plane $(n+1)$-gon with the set of vertices $M=\{0,1, \cdots, n\}$, as in Example 2.3.4. Let $\mathcal{T}$ be any triangulation of $P_{n+1}$. By lifting any triangle $\sigma \in \mathcal{T}$ with vertices $i, j, k$ to the triangle $\Delta^{\sigma} \subset \Delta^{n}$ with vertices $\{i\},\{j\},\{k\}$, we associate to $\mathcal{T}$ a 2-dimensional simplicial subset $\Delta^{\mathcal{T}} \subset \Delta^{n}$ homeomorphic to $P_{n+1}$.

We now recall the main definitions of [HSS1], the first one being a modification of that of Rezk [Rez01]. 
Definition 3.1.4. Let $\mathbf{C}$ be a combinatorial model category, and let $X \in \mathbf{C}_{\Delta}$ be a simplicial object.

(1) We say that $X$ is 1 -Segal if, for every $n \geq 1$, the morphism

$$
f_{n}: X_{n} \longrightarrow(I[n], X)_{R}=X_{1} \times{ }_{X_{0}}^{R} X_{1} \times_{X_{0}}^{R} \cdots \times{ }_{X_{0}}^{R} X_{1},
$$

induced by the embedding $I[n] \hookrightarrow \Delta^{n}$, is a weak equivalence in $\mathbf{C}$.

(2) We say that $X$ is 2 -Segal if, for every $n \geq 2$ and every triangulation $\mathcal{T}$ of $P_{n+1}$, the morphism

$$
f_{\mathcal{T}}: X_{n} \longrightarrow\left(\Delta^{\mathcal{T}}, X\right)_{R}
$$

induced by the embedding $\Delta^{\mathcal{T}} \hookrightarrow \Delta^{n}$, is a weak equivalence in $\mathbf{C}$.

Note the particular case when $\mathbf{C}$ is an ordinary category with trivial model structure. In this case the conditions involve underived membrane spaces and say that the corresponding morphisms $f_{n}$, resp. $f_{\mathcal{T}}$, are isomorphisms.

We now recall the path space criterion, a result from [HSS1] which is very useful to establish the 2-Segal property in many cases. For ordinals $I$ and $J$ their join is the set $I \sqcup J$ ordered so that each element of $I$ precedes each element of $J$. For a simplicial object $X$ its initial and final path spaces are the simplicial objects $P^{\triangleleft} X$ and $P^{\triangleright} X$ induced from $X$ by pullback along the endofunctors

$$
i, f: \Delta \longrightarrow \Delta, \quad i(I)=[0] * I, \quad f(I)=I *[0] .
$$

Theorem 3.1.5 (6.3.2 in [HSS1]). A simplicial object $X$ is 2-Segal if and only if both $P^{\triangleleft} X$ and $P^{\triangleright} X$ are 1-Segal.

A cyclic object $X$ in $\mathbf{C}$ will be called $p$-Segal, if $\varsigma^{*} X$ is a $p$-Segal simplicial object. We will say that a covariant functor $\Delta \rightarrow \mathbf{C}$ or $\Lambda \rightarrow \mathbf{C}$ is $p$-coSegal, if the corresponding contravariant functor with values in $\mathbf{C}^{\text {op }}$ is $p$-Segal.

\subsection{Examples of cyclic 2-Segal objects}

Example 3.2.1 (The cyclic nerve and the $\mathbb{Z}_{+}$-nerve). For a small category $\mathcal{C}$ its cyclic nerve $\mathrm{NC}(\mathrm{C})$, is the cyclic set defined by

$$
\mathrm{NC}_{n}(\mathcal{C})=\operatorname{Fun}\left(Q^{n}, \mathcal{C}\right)
$$

where $Q^{n}$ denotes the underlying category of the $\mathbb{Z}_{+}$-category from Example 2.1.3. In other words, $\mathrm{NC}_{n}(\mathcal{C})$ is the set of cyclic chains of morphisms

$$
x_{0} \rightarrow x_{1} \rightarrow x_{2} \rightarrow \cdots \rightarrow x_{n} \rightarrow x_{0}
$$

in $\mathcal{C}$. Similarly, for a small $\mathbb{Z}_{+}$-category $\mathcal{C}=(\mathcal{C}, w)$ its $\mathbb{Z}_{+}$-nerve $\mathrm{N}^{\mathbb{Z}_{+}}(\mathcal{C})$, is the cyclic set defined by

$$
\mathrm{N}_{n}^{\mathbb{Z}_{+}}(\varrho)=\operatorname{Fun}_{\mathbb{Z}_{+}}\left(Q^{n}, \varrho\right)
$$

In other words, $\mathrm{N}_{n}^{\mathbb{Z}_{+}}(\mathcal{C})$ is the set of cyclic chains of morphisms as above, which form a factorization of $w$, i.e., each composition $x_{i} \rightarrow \cdots \rightarrow x_{i}$ around the circle is equal to $w_{x_{i}}$. 
Proposition 3.2.2. (a) For any small category $\mathcal{C}$ the cyclic set NC $\mathrm{C}$ is unital 2-Segal.

(b) For any small $\mathbb{Z}_{+}$-category $\mathcal{C}$ the cyclic set $\mathrm{N}^{\mathbb{Z}_{+}} \mathcal{C}$ is unital 2-Segal.

Proof. The first statement is proved in [HSS1, Th. 3.2.3], the second statement is proved similarly.

We can now state the main result of this section. Consider the cocyclic object $\mathcal{E} \bullet$ in dgcat ${ }^{(2)}$ from Proposition 2.4.1. We equip dgcat $^{(2)}$ with the Morita model structure introduced in \$1.1, so that weak equivalences are Morita equivalences. We define a cyclic object $\mathcal{E}$. which is defined by the formula

$$
\mathcal{E}_{\bullet}: \Lambda^{\mathrm{op}} \rightarrow \operatorname{dgcat}^{(2)},\langle n\rangle \mapsto\left(\mathcal{E}^{\langle n\rangle^{*}}\right)^{\mathrm{op}}
$$

where $\langle n\rangle \mapsto\langle n\rangle^{*}$ denotes the cyclic duality from $\$ 2.5$. Note that, by the compatibility of cyclic and Morita duality established in Corollary [2.5.7, we have a levelwise Morita equivalence

$$
\varepsilon_{\bullet} \simeq R \underline{\operatorname{Hom}}\left(\varepsilon^{\bullet}, \operatorname{Perf}_{\mathbf{k}}^{(2)}\right)
$$

of cyclic objects in dgcat ${ }^{(2)}$.

Theorem 3.2.4. The following statements hold:

(a) The cocyclic object $\mathcal{E} \bullet$ in $\left(\mathrm{dgcat}^{(2)}, \mathrm{Mo}\right)$ is 2-coSegal.

(b) The cyclic object $\mathcal{E}_{\bullet}$ in $\left(\mathrm{dgcat}^{(2)}, \mathrm{Mo}\right)$ is 2-Segal.

Proof. From the adjunction (1.1.4), we deduce that $R \underline{\operatorname{Hom}}(-,-)$ maps homotopy colimits in the first variable to homotopy limits. Therefore, in light of (3.2.3), (b) follows immediately from (a). To show (a), we use Theorem 3.1 .5 to reduce to the statement that the cosimplicial objects $P^{\triangleleft}\left(\mathcal{E}^{\bullet}\right), P^{\triangleright}\left(\mathcal{\varepsilon}^{\bullet}\right)$ are 1 -coSegal. We consider the case of $P^{\triangleleft}\left(\mathcal{\varepsilon}^{\bullet}\right)$. By definition, we have

$$
\left(P^{\triangleleft}\left(\mathcal{E}^{\bullet}\right)\right)^{n}=\mathcal{E}^{n+1} .
$$

The key point of the argument is now that the Morita equivalences

$$
r_{n+1}^{\triangleleft}: \mathcal{A}^{n+1} \stackrel{\simeq}{\longrightarrow} \mathcal{E}^{n+1}
$$

from Proposition 2.4.6 assemble to give a weak equivalence of cosimplicial objects

$$
\mathcal{A}^{\bullet+1} \stackrel{\simeq}{\longrightarrow} P^{\triangleleft}\left(\varepsilon^{\bullet}\right)
$$

in $\left(\operatorname{dgcat}^{(2)}, \mathrm{Mo}\right)$ where the cosimplicial structure of $\mathcal{A}^{\bullet+1}$ is obtained in an obvious way with coface maps given by composing morphisms and codegeneracies by filling in identity morphisms. Therefore, it suffices to show that the cosimplicial object $\mathcal{A}^{\bullet+1}$ is 1 -Segal. Since homotopy fiber products in $\left(\mathrm{dgcat}^{(2)}\right)^{\text {op }}$ translate to homotopy pushouts in dgcat ${ }^{(2)}$ this amounts to verifying that, for every $n \geq 1$, the 1-coSegal map

$$
\operatorname{holim}\left\{\mathcal{A}^{2} \leftarrow \mathcal{A}^{1} \rightarrow \mathcal{A}^{2} \leftarrow \mathcal{A}^{1} \rightarrow \cdots \leftarrow \mathcal{A}^{1} \rightarrow \mathcal{A}^{2}\right\} \longrightarrow \mathcal{A}^{n}
$$

is a Morita equivalence. Since the maps $\mathcal{A}^{1} \rightarrow \mathcal{A}^{2}$ appearing in the homotopy colimit are cofibrations in $\left(\right.$ dgcat $\left.^{(2)}, \mathrm{Qe}\right)$ (and hence in $\left(\right.$ dgcat $\left.^{(2)}, \mathrm{Mo}\right)$ ), we may replace the homotopy colimit by an ordinary colimit. The resulting statement is clearly true. 


\subsection{Background on triangulated surfaces and ribbon graphs}

Here we collect some well-known material on surfaces and their triangulations. More details can be found in [FG06, FST08] and references therein.

\subsubsection{Marked oriented surfaces}

By a surface we mean a compact, connected, oriented 2-dimensional smooth manifold $S$ with boundary, denoted $\partial S$. We denote by $T^{2}, S^{2}$ and $D^{2}$ the 2-dimensional torus, sphere, and disk, respectively.

Definition 3.3.1. A stable marked surface is a pair $(S, M)$ where $S$ is a surface and $M \subset S$ is a nonempty finite subset of points such that:

(1) Each component of $\partial S$ contains at least one point from $M$.

(2) The following unstable cases are excluded:

(a) $S$ is diffeomorphic to $S^{2},|M| \leq 2$,

(b) $S$ is diffeomorphic to $D^{2},|M|=1$, or $|M|=2$ and $M \subset \partial S$.

In the sequel all marked surfaces will be assumed stable, unless indicated otherwise. For a marked surface $(S, M)$ we have the groups

$$
\operatorname{Diffeo}^{+}(S, M), \quad \mathfrak{G}(S, M)=\pi_{0} \operatorname{Diffeo}^{+}(S, M)
$$

of orientation preserving diffeomorphisms $S \rightarrow S$ preserving $M$ as a set, and of isotopy classes of such diffeomorphisms. The group $\mathfrak{G}(S, M)$ is known as the mapping class group of $(S, M)$.

Remark 3.3.2. It is often convenient to view interior marked points $x \in M-\partial S$ as punctures, by removing them to form the noncompact surface $S-(M-\partial S)$.

Further, when representing surfaces by Ribbon graphs (see \$3.3.4), it will be convenient to transform $(S, M)$ into a new compact surface where

(1) the interior marked points become closed boundary components,

(2) the marked points on $\partial S$ become closed intervals on the boundary.

The construction which naturally performs the modifications (1) and (2) is called the real blowup $S_{M}$ of $S$ along $M$ (An12, §2.1]), obtained by adding the set of inward tangent directions at each $x \in M$. It is further convenient, to form a noncompact surface by removing the open boundary intervals in the complement of the blown up marked boundary points creating open ends of the surface. For example, Figure 1 displays the marked surface given by a disk with one interior and one boundary marked point, as well as its real blowup which is an annulus with an open end on one of its boundary components. 


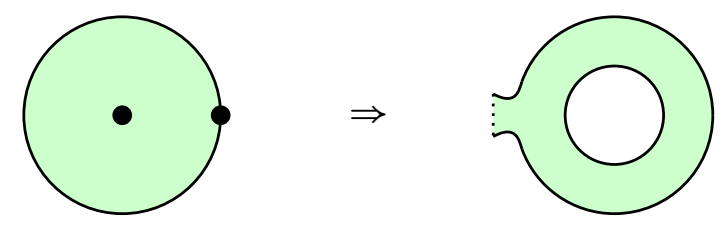

Figure 1: Disk with two marked points and corresponding real blowup

Example 3.3.3. As a simple but important case, our definition of a marked surface incudes $\left(P_{n+1}, M\right)$ where $P_{n+1}, n \geq 2$, is a convex $(n+1)$-gon in the plane, and $M$ is its set of vertices. Via a homeomorphism with the closed disk, this is a smooth manifold with boundary. We have $\mathfrak{G}\left(P_{n+1}, M\right)=\mathbb{Z} /(n+1)$.

Definition 3.3.4. A simple curve on a marked surface $(S, M)$ is a continuous map $\gamma$ : $[0,1] \rightarrow M$ with the following properties:

(1) The endpoints $\gamma(0), \gamma(1)$ lie in $M$. They can coincide.

(2) Except for possible coincidence of the endpoints, $\gamma$ does not intersect itself, nor $M$.

(3) If the endpoints coincide, $\gamma(0)=\gamma(1)=x$, then $\gamma$ gives a nontrivial element of the fundamental group $\pi_{1}(S-M \cup\{x\}, x)$.

A (simple) arc on $(S, M)$ is an equivalence class of simple curves under isotopies and reversal of parametrization. We denote by $\mathbb{A}(S, M)$ the set of arcs. An oriented arc on $(S, M)$ is an equivalence class of simple curves under isotopies. We denote by $\Delta(S, M)$ the set of oriented arcs.

This definition differs from [FST08, Def. 2.2] in that we allow, as arcs, segments joining adjacent marked points on the same boundary component. This does not affect the validity of the results we need, while allowing for a more suggestive interpretation of $\Delta(S, M)$, as the following examples show.

Examples 3.3.5. (a) In the situation of Example 3.3.3, $\Delta\left(P_{n+1}, M\right)$ is identified with the root system of type $A_{n}$.

(b) Let $S=T^{2}$ be a torus and $M$ consist of one point, denoted 0 . An oriented arc $\gamma$ has a homology class $[\gamma] \in H_{2}\left(T^{2}, \mathbb{Z}\right)=\mathbb{Z}^{2}$ which must be a primitive vector of the lattice $\mathbb{Z}^{2}$. Thus $\Delta(S, M)$ is identified with the set of primitive vectors in $\mathbb{Z}^{2}$, and $\mathbb{A}(S, M)$ is identified with $\mathbb{P}^{1}(\mathbb{Q})$.

\subsubsection{Triangulations as systems of arcs}

Two arcs are called compatible if there are simple curves representing them which do not intersect in $S-M$. It is known [FST08, Prop. 2.5] that any collection of pairwise 

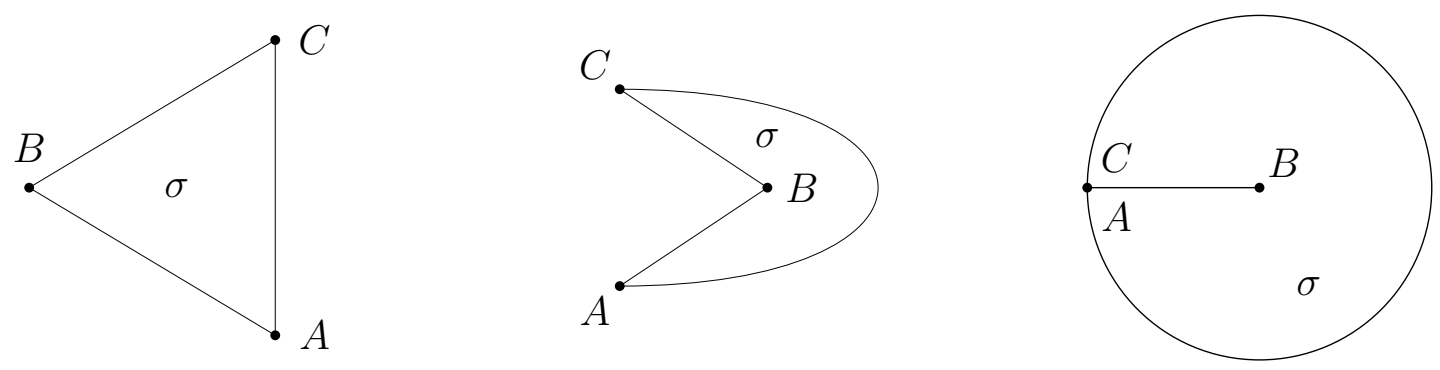

Figure 2: $\operatorname{Vert}(\sigma)=\{A, B, C\}$ in all cases.

compatible arcs can be represented by a collection of simple curves which pairwise do not intersect in $S-M$. An ideal triangulation of $(S, M)$ is defined as a maximal collection of pairwise compatible arcs.

Pairwise non-intersecting curves from a maximal collection cut $S$ into "ideal triangles with vertices in $M$ ", which are regions $\sigma$ diffeomorphic to the interior of the standard plane triangle $P_{3}$. Each such $\sigma$ comes with a canonical 3-element set Vert $(\sigma)$ of "intrinsic vertices" (or "corners") which is equipped with a cyclic order via the orientation of $S$. Note that different elements of $\operatorname{Vert}(\sigma)$ may correspond to the same element of $M$, i.e., the vertices (and even edges) of a triangle can become identified, see Fig 3.3.2. Similarly, each $\operatorname{arc} a$ comes with a 2 -element set $\operatorname{Vert}(a)$ of intrinsic vertices ("half-edges") which can become identified in $S$, if $a$ is a loop.

\subsubsection{Triangulations, tesselations, and spanning graphs}

An ideal triangulation $\mathcal{T}$ can be encoded by its dual graph $\Gamma_{\mathcal{T}}$ obtained by putting one vertex $v_{\sigma}$ inside each triangle $\sigma$ and joining the $v_{\sigma}$ by edges corresponding to common edges of the triangles, see (0.5). As this construction allows a uniform treatment of all degenerate cases, we recall precise definitions.

Definition 3.3.6. (a) A graph $\Gamma$ is a finite, 1-dimensional $\mathrm{CW}$-complex without isolated points. For a vertex $v \in \operatorname{Vert}(\Gamma)$ we denote $\operatorname{Ed}(v)$ the set of germs of edges at $v$ (a loop beginning and ending at $v$ gives rise to two germs of edges at $v$ ). The cardinality of $\operatorname{Ed}(v)$ is called the valency of $v$. The set of 1 -valent vertices is denoted by $\partial \Gamma$. A graph is called 3 -valent if all vertices have valence 1 or 3 .

(b) Let $(S, M)$ be a marked surface. A spanning graph for $(S, M)$ is an embedded graph $\Gamma \subset S-M$ such that $\partial \Gamma \subset \partial S$ and both maps

$$
\Gamma \longrightarrow S-M, \quad \partial \Gamma \longrightarrow \partial S-M
$$

are homotopy equivalences.

Proposition 3.3.7. Let $(S, M)$ be a marked surface. Forming the dual graph defines a bijection $\mathcal{T} \mapsto \Gamma_{\mathcal{T}}$ between ideal triangulations of $(S, M)$ and isotopy classes of 3-valent spanning graphs for $(S, M)$. 
We will further call a tessellation of $(S, M)$ an isotopy class of (not necessarily 3valent) spanning graphs $\Gamma$ for $(S, M)$. Each such $\Gamma$ encodes a decomposition of $S$ into curvilinear polygons with vertices in $M$, one polygon for each vertex $v \in \Gamma$ of valence $\geq 3$.

\subsubsection{Ribbon graphs, Stasheff polytopes, and the tessellation complex}

A ribbon graph is a graph $\Gamma$ together with a choice of a total cyclic order on each set $\operatorname{Ed}(v), v \in \operatorname{Vert}(\Gamma)$. As any graph embedded into an oriented surface, a spanning graph for $(S, M)$ has a natural ribbon structure.

Conversely, a ribbon graph $\Gamma$ gives rise to an oriented surface with boundary $\Sigma_{\Gamma}$ as follows. Each vertex $v$ of $\Gamma$ corresponds to a ribbon corolla as illustrated in Figure 3 , Further, each edge $e$ of $\Gamma$ corresponds to a ribbon strip as illustrated in Figure 4. The

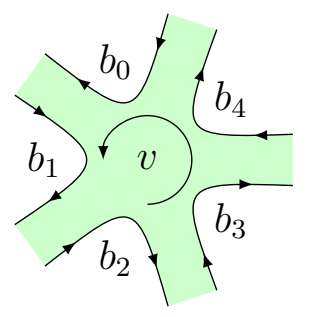

Figure 3: Ribbon corolla corresponding to a vertex $v$ of $\Gamma$ of valency 5

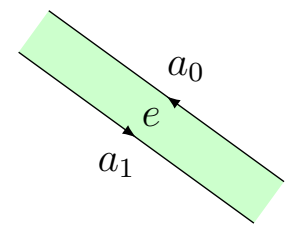

Figure 4: Ribbon strip corresponding to an edge $e$ of $\Gamma$

ribbon strips are then glued to the ribbon corollas according to the incidence relations provided by $\Gamma$. As a result of this procedure, we obtain an oriented surface with boundary which we denote by $\Sigma_{\Gamma}$. See, e.g., [Pen10], Ch. 1, $\S 1.3$ for the case of graphs without 1 -valent vertices. If $\Gamma$ does have 1 -valent vertices, they give rise to open ends at the boundary of $\Sigma_{\Gamma}$. If $\Gamma$ is a spanning graph for a stable marked surface $(S, M)$, then $\Sigma_{\Gamma}$ is diffeomorphic to the real blowup $\widetilde{S}_{M}$ from Remark 3.3.2.

Note that for a ribbon tree $T$ we have a canonical cyclic order on $\partial T$, since in this case the ribbon structure gives an isotopy class of embeddings into $\mathbb{R}^{2}$. Let $\Gamma, \Gamma^{\prime}$ be ribbon graphs. A contraction $p: \Gamma \rightarrow \Gamma^{\prime}$ is a surjective cellular map such that for any vertex $v^{\prime} \in \Gamma^{\prime}$ the preimage $p^{-1}\left(v^{\prime}\right)$ is a sub-tree in $\Gamma$, and the induced map $\partial\left(p^{-1}\left(v^{\prime}\right)\right) \rightarrow \operatorname{Ed}\left(v^{\prime}\right)$ is a bijection preserving the cyclic order.

We denote by $K_{n}$ the $n$th Stasheff polytope. Thus, the vertices of $K_{n}$ are in bijection with the following three canonically identified sets:

(S) Bracketings of a product of $n$ factors.

(S') Triangulations of the $(n+1)$-gon $P_{n+1}$.

$\left(\mathrm{S}^{\prime \prime}\right)$ Planar 3-valent trees with $(n+1)$ "tails" (1-valent vertices) labelled cyclically from 0 to $n$. 
The bijection between $\left(\mathrm{S}^{\prime}\right)$ and $\left(\mathrm{S}^{\prime \prime}\right)$ is a particular case of Proposition 3.3.7. More generally, faces of $K_{n}$ of arbitrary dimension are labelled by planar trees with the same tails as in ( $\mathrm{S}^{\prime}$ ) but not necessarily 3-valent. For instance, edges correspond to trees with one 4-valent vertex and the rest being 3- (or 1-)valent. The maximal face corresponds to a "corolla" (tree with one vertex of valency $n+1$ ). Note that both $\left(\mathrm{S}^{\prime}\right)$ and (S") allow for the definition of $K_{I}$ for any finite cyclic ordinal $I$, with $K_{n}$ corresponding to $I=\langle n\rangle$. We put $K_{I}=$ pt for $|I| \leq 3$.

For a ribbon graph $\Gamma$, we define its Stasheff polytope as

$$
K_{\Gamma}=\prod_{v \in \operatorname{Vert}(\Gamma)} K_{\operatorname{Ed}(v)}
$$

A contraction $p: \Gamma \rightarrow \Gamma^{\prime}$ gives rise to an embedding $k_{p}: K_{\Gamma} \hookrightarrow K_{\Gamma^{\prime}}$, which realizes $K_{\Gamma}$ as a face of $K_{\Gamma^{\prime}}$.

Let now $(S, M)$ be an arbitrary marked surface and denote by $\Sigma(S, M)$ the set if isotopy classes of all, not necessarily 3-valent, spanning graphs for $(S, M)$. This set is partially ordered by degeneration: we say that $\Gamma \leq \Gamma^{\prime}$, if $\Gamma^{\prime}$ can be obtained from $\Gamma$ by collapsing some edges to points. In this case, the subgraph shrunk to each vertex of $\Gamma^{\prime}$ is a tree, so that we have a contraction $\Gamma \rightarrow \Gamma^{\prime}$ and the corresponding face embedding $K_{\Gamma} \hookrightarrow K_{\Gamma^{\prime}}$.

Definition 3.3.8. The tessellation complex $K_{S, M}$ is a CW-complex glued from the cells $K_{\Gamma}, \Gamma \in \Sigma(S, M)$, using the face identifications induced by degenerations.

In particular, vertices of $K_{S, M}$ correspond to ideal triangulations of $(S, M)$, edges correspond to "flips" on 4-gons, as in Example 0.2, and so on. More precisely, a cell of $K_{S, M}$, i.e., an isotopy class of spanning graphs $\Gamma$, can be seen as encoding a tessellation of $(S, M)$, i.e., a decomposition of $S$ into curvilinear polygons with vertices in $M$, see [Pen10], Ch.1, Th. 1.25.

The mapping class $\mathfrak{G}(S, M)$ acts on $K_{S, M}$ by automorphisms. Crucial for us will be the following result due to Harer (Theorem 1.1 and Theorem 2.1 in [Har86]).

Proposition 3.3.9. The $C W$-complex $K_{S, M}$ is contractible.

\subsection{Cyclic membrane spaces}

Let $\mathbf{C}$ be a combinatorial model category. Given a cyclic object $X \in \mathbf{C}_{\Lambda}$ we define, for every cyclic set $D \in \mathcal{S}_{e} t_{\Lambda}$, the cyclic membrane space to be

$$
((D, X))=\lim _{\left\{\Lambda^{n} \rightarrow D\right\}}^{\mathbf{C}} X_{n},
$$

As in the simplicial case, this construction can be expressed in terms of the right Kan extension $\Upsilon_{*}: \mathbf{C}_{\Lambda} \longrightarrow \mathbf{C}_{\mathcal{S e t}_{\Lambda}}$ along the Yoneda embedding $\Upsilon: \Lambda \rightarrow \mathcal{S}_{\text {et }}$, so that we have a natural isomorphism

$$
((D, X)) \cong\left(\Upsilon_{*} X\right)(D)
$$


Again, we can derive these constructions, defining the derived cyclic membrane space to be

$$
((D, X))_{R}=\operatorname{holim}_{\left\{\Lambda^{n} \rightarrow D\right\}}^{\mathbf{C}} X_{n},
$$

obtaining the description

$$
((D, X))_{R} \simeq\left(R \Upsilon_{*} X\right)(D)
$$

in terms of the right homotopy Kan extension along the Yoneda embedding. In particular, we have $((D, X))_{R} \simeq((D, \widetilde{X}))$, where $X \rightarrow \widetilde{X}$ is an injectively fibrant replacement of $X$.

\subsubsection{From a triangulated surface to a cyclic membrane}

Let $(S, M)$ be a stable marked surface, and let $\mathcal{T}$ be a triangulation of $(S, M)$. We denote by $\mathcal{T}_{1}$ and $\mathcal{T}_{2}$ the set of arcs and triangles of $\mathcal{T}$. Recall from $\$ 3.3 .2$ that each arc $a \in \mathcal{T}_{1}$ has a 2-element set Vert $(a)$ of "intrinsic endpoints", which, as any 2-element set, can be canonically considered as a cyclic ordinal. Recall further that each triangle $\sigma \in \mathcal{T}_{2}$ has a 3-element set Vert $(\sigma)$ of "intrinsic vertices" which is made into a cyclic ordinal by the orientation of $S$. Whenever an $\operatorname{arc} a$ is a side of a triangle $\sigma$ (notation $a \subset \sigma$ ), we have an embedding $u_{a, \sigma}: \operatorname{Vert}(a) \rightarrow \operatorname{Vert}(\sigma)$ which can be considered as a morphism in the category $\boldsymbol{\Lambda}$. In particular, we have an embedding of the cyclic simplices

$$
\left(u_{a, \sigma}\right)_{*}: \Lambda^{\operatorname{Vert}(a)} \longrightarrow \Lambda^{\operatorname{Vert}(\sigma)} .
$$

Let $\mathcal{T}_{[1,2]}$ be the incidence category of $\mathcal{T}$, with the set of objects being $\mathcal{T}_{1} \sqcup \mathcal{T}_{2}$, and nonidentity morphisms given by inclusions $a \subset \sigma$. Let $U_{\mathcal{T}}: \mathcal{T}_{[1,2]} \rightarrow \operatorname{Set}_{\Lambda}$ be the functor sending:

- an object $a$ to $\Lambda^{\operatorname{Vert}(a)}$,

- an object $\sigma$ to $\Lambda^{\operatorname{Vert}(\sigma)}$,

- a morphism $a \subset \sigma$ to the morphism $\left(u_{a, \sigma}\right)_{*}$.

The cyclic membrane corresponding to $\mathcal{T}$ is defined as the colimit

$$
\Lambda^{\mathcal{T}}=\lim \operatorname{Set}_{\Lambda} U_{\mathcal{T}}
$$

mimicking the way $S$ itself is glued out of triangles $\sigma \in \mathcal{T}_{2}$ identified along arcs $a \in \mathcal{T}_{1}$.

Remark 3.4.2. The geometric realization $\left|\Lambda^{\mathcal{T}}\right|$ of (the simplicial set corresponding to) the cyclic set $\Lambda^{\mathcal{T}}$ is a 3 -dimensional manifold with boundary. As showed in [DHK85], the realization of any cyclic set has a natural $S^{1}$-action. In our case $\left|\Lambda^{\mathcal{T}}\right|$ is an $S^{1}$-bundle over $S$ which is obtained from the tangent circle bundle by performing a surgery at each point of $M$ (in particular, if $S$ is a compact surface of genus $g$, then the degree of the bundle is $2-2 g-|M|)$. Among other things, this means that $\left|\Lambda^{\mathcal{T}}\right|$ is independent on $\mathcal{T}$, 
up to homeomorphism. If $S$ is equipped with a holomorphic structure, then $\left|\Lambda^{\mathcal{T}}\right|$ can be identified with the circle bundle corresponding to the holomorphic line bundle

$$
T_{S}(\log M)=\left(\Omega_{S}^{1}(\log M)\right)^{*}
$$

whose sections are holomorphic vector fields on $S$ vanishing on $M$. This fact can be obtained by carefully analyzing the case when $S$ is a triangle (with a complex structure) and $M$ is the set of its 3 vertices. In this case by [Dr04, the interior of $\left|\Lambda^{M}\right|=\left|\Lambda^{2}\right|$ is the space of cyclically monotone embeddings $M \rightarrow S^{1}$. The Riemann Mapping Theorem identifies this space with the space of biholomorphisms $f$ from $S$ to the unit disk $D=$ $\{|z| \leq 1\}$ (such $f$ is uniquely determined by the images of three points on the boundary). Another way of determining $f$ is by prescribing an interior point $s \in S$ (sent by $f$ to 0 ) and a tangent direction at $s$ (sent by $d_{s} f$ to the tangent direction of $\mathbb{R}_{+}$at 0 ). This provides an identification of the interior of $\left|\Lambda^{M}\right|$ with the tangent circle bundle of the interior of $S$. We omit further details.

\subsubsection{From a ribbon graph to a cyclic membrane}

We provide a dual description of the association $\mathcal{T} \mapsto \Lambda^{\mathcal{T}}$ in terms of ribbon graphs which easily allows us to generalize it to more general polygonal subdivisions.

Let $\Gamma$ be a ribbon graph. For a vertex $v$ of $\Gamma$ let $B(v)$ be the set of oriented arcs comprising the local boundary of the ribbon corolla of $v$. See Figure 3 where this set is denoted $\left\{b_{0}, \ldots, b_{n}\right\}$. Note that $B(v)$ has a natural cyclic order inherited from that on $\operatorname{Ed}(v)$, the set of half-edges edges incident to $v$. More precisely, $B(v)=\operatorname{Ed}(v)^{*}$ is the set of interstices in $\operatorname{Ed}(v)$. We denote by $\Lambda^{B(v)}$ the cyclic simplex corresponding to $B(v)$.

For an edge $e$ of $\Gamma$, let $B(e)$ be the 2-element set of components of the ribbon strip $e$. See Figure 4 where this set is denoted $\left\{a_{0}, a_{1}\right\}$. Invariantly, $B(e)=\operatorname{Vert}(e)^{*}$, where Vert $(e)$ is the 2-element set of endpoints of $e$ (considered distinct even if $e$ is a loop). As any 2-element set, $B(e)$ has a unique total cyclic order. We associate to $e$ the cyclic 1-simplex $\Lambda^{B(e)}$.

For a flag $(v, e)$ consisting of a vertex and an edge of $\Gamma$, we have an inclusion of cyclic ordinals $u_{v, e}: B(e) \rightarrow B(v)$ and the corresponding embedding of the cyclic simplexes

$$
\left(u_{v, e}\right)_{\star}: \Lambda^{B(e)} \rightarrow \Lambda^{B(v)} .
$$

Let $\Gamma_{[0,1]}$ be the incidence category of $\Gamma$ with the set of objects being $\operatorname{Vert}(\Gamma) \sqcup \operatorname{Ed}(\Gamma)$ and morphisms being incidence inclusions. As before, we get a functor $U_{\Gamma}: \Gamma_{[0,1]}^{\mathrm{op}} \rightarrow \mathcal{S e t}_{\Lambda}$ sending $e$ to $\Lambda^{B(e)}, v$ to $\Lambda^{B(v)}$ and an incidence $v \subset e$ to $u_{v, \text { e* }}$. We define the cyclic membrane corresponding to $\Gamma$ as

$$
\Lambda^{\Gamma}=\underline{\lim }^{\operatorname{Set}_{\Lambda}} U_{\Gamma}
$$

Example 3.4.3. If $\mathcal{T}$ is a triangulation of $(S, M)$ and $\Gamma$ is its dual ribbon graph, then $\operatorname{Vert}(\Gamma)=\mathcal{T}_{2}, \operatorname{Ed}(\Gamma)=\mathcal{T}_{1}$, the functor $U_{\Gamma}$ is identified with $U_{\mathcal{T}}$, and $\Lambda^{\Gamma}$ with $\Lambda^{\mathcal{T}}$. 
Let $p: \Gamma \rightarrow \Gamma^{\prime}$ be a contraction of ribbon graphs. For any vertex $v^{\prime}$ of $\Gamma$, we have a canonical map

$$
\coprod_{v \in p^{-1}\left(v^{\prime}\right)} \Lambda^{B(v)} \rightarrow \Lambda^{B\left(v^{\prime}\right)}
$$

of cyclic sets. These maps induce an inclusion of cyclic membranes $\Lambda^{\Gamma} \rightarrow \Lambda^{\Gamma^{\prime}}$. Since this association is functorial, we obtain the following result.

Proposition 3.4.4. The cyclic membrane construction

$$
\mathcal{R} i b \longrightarrow \mathcal{S e t}_{\Lambda}, \Gamma \mapsto \Lambda^{\Gamma}
$$

extends to a functor on the category of Ribbon graphs with contractions as morphisms.

\subsubsection{Mapping class group actions}

Let $X \in \mathbf{C}_{\Lambda}$ be a cyclic object and $\Gamma$ a Ribbon graph. We define

$$
R X_{\Gamma}:=\left(\left(\Lambda^{\Gamma}, X\right)\right)_{R}
$$

where $\Lambda^{\Gamma}$ denotes the cyclic membrane corresponding to $\Gamma$. Similarly, given a marked surface $(S, M)$ and a triangulation $\mathcal{T}$ of $S$ with set of vertices $M$, we denote by $R X_{\mathcal{T}}=$ $\left(\left(\Lambda^{\Gamma}, X\right)\right)_{R}$ the corresponding derived membrane space.

Theorem 3.4.5. Let $X$ be a cyclic 2-Segal object in $\mathbf{C}_{\Lambda}$. Then the functor

$$
R X: \mathcal{R} i b \longrightarrow \mathcal{C}, \Gamma \mapsto R X_{\Gamma}
$$

maps contractions of ribbon graphs to weak equivalences in $\mathbf{C}$.

Proof. It suffices to show that a contraction $p: \Gamma \rightarrow \Gamma^{\prime}$ of a single edge $e$ of $\Gamma$ to a vertex $v$ of $\Gamma^{\prime}$ induces a weak equivalence. Without restriction we assume that $X$ is injectively fibrant. Assume that the edge $e$ is incident to vertices of valency $m+1$ and $n+1$, respectively. Then the map of cyclic membranes $\Lambda^{\Gamma} \rightarrow \Lambda^{\Gamma^{\prime}}$ induced by $p$ is a pushout of the map

$$
\Lambda^{\{0, n, \ldots, n+m\}} \coprod_{\Lambda\{0, n\}} \Lambda^{\{0,1, \ldots, n\}} \rightarrow \Lambda^{\{0,1, \ldots, n+m\}} .
$$

Evaluating $X$ on this map, we obtain

$$
X_{\{0,1, \ldots, n+m\}} \rightarrow X_{\{0, n, \ldots, n+m\}} \times_{X_{\{0, n\}}} X_{\{0,1, \ldots, n\}}
$$

which is the 2-Segal map corresponding to the subdivision of a $(n+m+1)$-gon into a $(n+1)$-gon and a $(m+1)$-gon along the edge $\{0, n\}$. Thus it is a weak equivalence, and hence, due to the fibrancy assumption, a trivial fibration in C. Since the map

$$
R X_{\Gamma} \rightarrow R X_{\Gamma^{\prime}}
$$

induced by $p$ is a pullback of the above 2-Segal map it is a weak equivalence as well. 
Let $(S, M)$ be a marked surface and let $\Sigma(S, M)$ be the partially ordered set of isotopy classes of spanning graphs for $(S, M)$ where, as in $\$ 3.3$, the order is given by degeneration. The geometric realization $|\Sigma(S, M)|$ of the poset $\Sigma(S, M)$ is homeomorphic to the tessellation complex $K_{S, M}$ from \$3.3.4, and hence contractible by Proposition 3.3.9. We obtain the following immediate consequences.

Corollary 3.4.6. Let $X$ be a cyclic 2-Segal object in $\mathbf{C}$ and let $(S, M)$ be a stable marked surface. Then the object $R X_{\Gamma}, \Gamma \in \Sigma(S, M)$, is, up to weak equivalence, independent on the choice of $\Gamma$ and defines therefore a unique isomorphism class of objects in $\mathrm{Ho}(\mathbf{C})$ depending only on $(S, M)$.

Proof. This follows from Theorem 3.4.5, since $|\Sigma(S, M)|$ is connected.

Corollary 3.4.7. Let $X$ be a cyclic 2 -Segal object in $\mathbf{C}$ and let $(S, M)$ be a stable marked surface. The diagram

$$
\Sigma(S, M) \rightarrow \operatorname{Ho}(\mathbf{C}), \Gamma \mapsto R X_{\Gamma}
$$

admits a colimit, denoted by $R X_{(S, M)}$, which is, for every $\Gamma \in \Sigma(S, M)$, equipped with a canonical isomorphism $R X_{\Gamma} \cong R X_{(S, M)}$ in $\mathrm{Ho}(\mathbf{C})$. The mapping class group $\mathfrak{G}(S, M)$ acts on $R X_{(S, M)}$ by automorphisms in $\mathrm{Ho}(\mathbf{C})$.

Proof. This follows from Theorem 3.4.5 and the fact that $|\Sigma(S, M)|$ is connected and simply connected.

Definition 3.4.8. We call the object $R X_{(S, M)}$ the derived membrane space of the surface $(S, M)$ in $X$.

Remark 3.4.9. In the above results, we have only used the 1-connectedness of $|\Sigma(S, M)|$. The contractibility of $|\Sigma(S, M)|$ amounts to the statement that there exists a coherent action of the mapping class group. One way to make this precise for closed surfaces is to consider the full subcategory $\mathcal{R} i b_{3}$ of $\mathcal{R} i b$ spanned by stable, connected Ribbon graphs with each vertex of valence $\geq 3$. It is well-known (see, e.g., Igu02]), that we have a weak equivalence of topological spaces

$$
\left|\mathrm{N}\left(\mathcal{R} i b_{3}\right)\right| \simeq \coprod_{(S, M)} B \mathfrak{G}(S, M)
$$

where $(S, M)$ ranges over stable closed marked surfaces. Theorem 3.4 .5 implies that the functor

$$
\mathcal{R} i b \longrightarrow \mathbf{C}, \Gamma \mapsto R X_{\Gamma}
$$

maps all morphisms in $\mathcal{R} i b$ to weak equivalences. Passing to nerves, we obtain a map

$$
|\mathrm{N}(\mathcal{R} i b)| \longrightarrow|\mathrm{N}(W)|
$$

where $W$ denotes the subcategory of weak equivalences in $\mathbf{C}$. This map encodes, for each stable marked surface $(S, M)$, the choice of an object of $\mathbf{C}$ together with a coherent action of the mapping class group $\mathfrak{G}(S, M)$. A more refined analysis in the context of $\infty$-categories will be given in [HSS2]. 


\section{Application: Fukaya categories}

\subsection{Topological Fukaya categories}

We apply the theory of cyclic membrane spaces to the cocyclic 2-coSegal object $\mathcal{E}=\mathcal{E}^{\bullet}$ from Theorem 3.2.4, considered as a cyclic 2-Segal object in $\left(\text { dgcat }^{(2)}, \mathrm{Mo}\right)^{\mathrm{op}}$. We use the upper index notation $L \mathcal{E}^{\Gamma}$ to denote $R\left(\mathcal{E}^{\mathrm{op}}\right)_{\Gamma}$, and similarly for other types of derived membrane spaces. In particular, we write $L \mathcal{E}^{(S, M)}$ for $R\left(\mathcal{E}^{\mathrm{op}}\right)_{(S, M)}$ from Definition 3.4.8.

Definition 4.1.1. Let $(S, M)$ be a stable marked oriented surface. We call the derived membrane object

$$
\mathscr{F}^{(S, M)}=L \mathcal{E}^{(S, M)}
$$

the topological coFukaya category of $(S, M)$. Given a 2-periodic perfect dg-category $\mathcal{A}$, we call

$$
R \underline{\operatorname{Hom}}\left(\mathscr{F}^{(S, M)}, \mathcal{A}\right)
$$

the topological Fukaya category of $(S, M)$ with coefficients in $\mathcal{A}$. We introduce special notation for the Morita dual

$$
\mathscr{F}_{(S, M)}=R \underline{\operatorname{Hom}}\left(\mathscr{F}^{(S, M)}, \operatorname{Perf}_{\mathbf{k}}^{(2)}\right)
$$

which is simply called the topological Fukaya category of $(S, M)$.

As immediate consequences of the general theory of derived cyclic membranes, we obtain the following main results.

Theorem 4.1.2. Let $(S, M)$ be a stable marked oriented surface, and let $\Gamma$ be a spanning graph for $(S, M)$. Then we have canonical isomorphisms in $\mathrm{Hmo}^{(2)}$

$$
\begin{aligned}
& \mathscr{F}^{(S, M)} \simeq L \mathcal{E}^{\Gamma} \simeq \operatorname{holim}_{\left\{\Lambda^{n} \rightarrow \Lambda^{\Gamma}\right\}} \mathcal{E}^{n} \\
& \mathscr{F}_{(S, M)} \simeq R \mathcal{E}_{\Gamma} \simeq \operatorname{holim}_{\left\{\Lambda^{n} \rightarrow \Lambda^{\Gamma}\right\}} \mathcal{E}_{n},
\end{aligned}
$$

where the homotopy limits are taken in $\left(\mathrm{dgcat}^{(2)}, \mathrm{Mo}\right)$.

Proof. Corollary 3.4 .7

Therefore, while the definition of the topological (co)Fukaya category does not depend on any choice of a triangulation of $(S, M)$, we may chose a triangulation to compute it via the descent isomorphisms of Theorem 4.1.2.

Theorem 4.1.3. Let $(S, M)$ be a stable marked oriented surface. The topological (co)Fukaya categories $\mathscr{F}^{(S, M)}$ and $\mathscr{F}_{(S, M)}$ admit a canonical action of the mapping class group of $(S, M)$ via automorphisms in $\mathrm{Hmo}^{(2)}$.

Proof. Corollary 3.4 .7 
The name "topological Fukaya category" for $\mathscr{F}_{(S, M)}$ is justified as follows. First, if $(S, M)=(\sigma, \operatorname{Vert}(\sigma))$ is a triangle, then elements of the cyclic set $M^{*}$ are identified with edges of the triangle $\sigma$. For two such edges $i, j \in M^{*}$ the indecomposable object $E_{i j} \in \mathcal{E}_{M}=\mathcal{E}^{M^{*}}$ is then visualized by an oriented simple arc $\alpha$ beginning at an interior point of the edge $i$ and ending at an interior point of the edge $j$ (such arcs form one isotopy class). Let us denote this object $E_{\alpha}$.

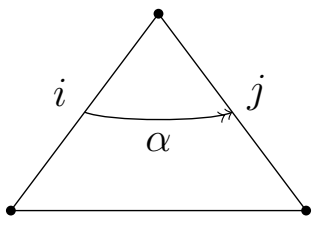

Next, let $\mathcal{T}$ be a triangulation of a stable marked surface $(S, M)$, and $\Gamma$ be the corresponding spanning graph. The homotopy limit defining $R \mathcal{E}_{\mathcal{T}}=R \mathcal{E}_{\Gamma}$ can be computed by reducing it to a homotopy fiber product which is then computed by using the concept of the path object $P(\mathcal{A}) \rightarrow \mathcal{A} \times \mathcal{A}$ of a dg category $\mathcal{A}$ from [Tab07], see the proof of Proposition 2.4.7. Explicitly, this means that local arcs in individual triangles as above can be combined, in the homotopy limit, yielding a large supply of aggregate objects:

(1) open Lagrangians: isotopy classes of oriented immersed $\operatorname{arcs} \beta$ which begin and end on $\partial S$ and avoid $M$. In the real blowup picture they correspond to arcs beginning and ending on open ends of the blown up surface.

(2) closed Lagrangians: isotopy classes of oriented closed immersed curves $\beta$ avoiding $\partial S \cup M$ and equipped with a flat $\mathbf{k}^{*}$-principal bundle (completely classified by its monodromy). In the real blowup picture $\beta$ is a closed oriented curve inside the blown up surface.

More precisely, the object $E_{\beta}$ corresponding to an arc or curve $\beta$ as above is obtained by gluing together the objects $E_{\alpha} \in \mathcal{E}_{\sigma}$ for $\sigma$ being a triangle of $\mathcal{T}$ and $\alpha$ being a component of $\beta \cap \sigma$. The monodromy for closed curves appears because of the $\mathbf{k}^{*}$-freedom in identifying the images of $E_{\alpha}$ and $E_{\alpha^{\prime}}$ in $\mathcal{E}_{\operatorname{Vert}(b)}$ for adjacent parts $\alpha, \alpha^{\prime}$ of $\beta$ cut by adjacent triangles with common edge $b$.

\subsection{Examples}

As an illustration, we show how our construction relates to some examples from Kontsevich's list [Kon09', Pictures]. In each case we first exhibit the surface Postnikov system generating the coFukaya category $\mathscr{F}(S, M)$, then refine this to a homotopy colimit presentation via Theorem 4.1.2, and, finally, explain how to identify $\mathcal{F}^{(S, M)}$ or $\mathcal{F}_{(S, M)}$ with an algebro-geometric derived category. We assume the ground field $\mathbf{k}$ to be algebraically closed. 


\subsubsection{The affine line}

Let $(S, M)$ be a disk with one interior and one boundary marked point. The Ribbon graph $\Gamma$ displayed in Figure 5 is a spanning graph in $(S, M)$ whose corresponding real blowup is given by an annulus with an open end on one of its boundary components.
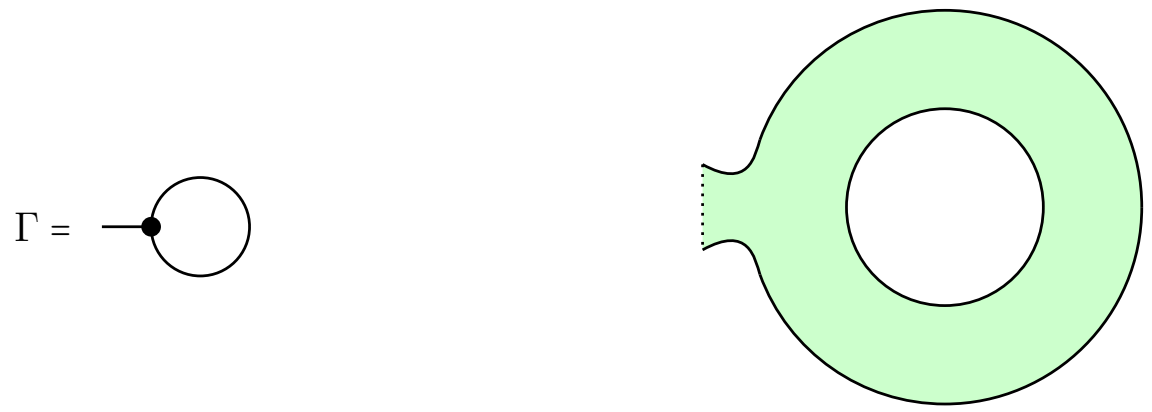

Figure 5: Ribbon graph $\Gamma$ with corresponding real blowup

\section{Surface Postnikov system:}

$$
A \stackrel{\alpha}{\longrightarrow} A \longrightarrow C \longrightarrow \Sigma A
$$

(One distinguished triangle with two terms being the same.)

\section{Homotopy colimit presentation:}

$$
\mathscr{F}(S, M) \simeq \mathcal{E}^{2} \coprod_{\mathcal{E}^{1} \amalg \mathcal{E}^{1}}^{\mathrm{h}} \mathcal{E}^{1} \simeq \mathcal{A}^{2} \coprod_{\mathcal{A}^{1} \amalg \mathcal{A}^{1}}^{\mathrm{h}} \mathcal{A}^{1} \simeq \mathcal{A}^{2} \coprod_{\mathcal{A}^{1} \amalg \mathcal{A}^{1}} \mathcal{A}^{1}=: \mathcal{L}
$$

where we use the Morita equivalences $\mathcal{A}^{n} \rightarrow \mathcal{E}^{n}$ to simplify the homotopy pushout and then identify it with the usual pushout using the fact that the map $\mathcal{A}^{1} \amalg \mathcal{A}^{1} \rightarrow \mathcal{A}^{2}$ is a Morita cofibration. The usual push out, denoted $\mathcal{L}$, is the $\mathbb{Z} / 2 \mathbb{Z}$-graded $\mathbf{k}$-linear category freely generated by the quiver with one vertex and one loop. It corresponds to the part $\{A \stackrel{\alpha}{\rightarrow} A\}$ in (4.2.1).

Algebro-geometric picture: Passing to the perfect envelope we obtain

$$
\mathscr{F}^{(S, M)} \simeq \operatorname{Perf}_{\mathcal{L}}^{(2)} \simeq \operatorname{Perf}^{(2)}\left(\mathbb{A}_{\mathbf{k}}^{1}\right),
$$

the $\mathbb{Z} / 2 \mathbb{Z}$-folded category of perfect complexes on the affine line. Dually, we obtain the category

$$
\mathscr{F}_{(S, M)} \simeq R \underline{\operatorname{Hom}}\left(\operatorname{Perf}_{\mathcal{L}}^{(2)}, \operatorname{Perf}_{\mathrm{k}}^{(2)}\right) \simeq R \underline{\operatorname{Hom}}\left(\mathcal{L}, \operatorname{Perf}_{\mathrm{k}}^{(2)}\right)
$$

which can be identified with the full subcategory of $\operatorname{Perf}^{(2)}\left(\mathbb{A}_{\mathbf{k}}^{1}\right)$ consisting of those complexes whose cohomology is compactly supported.

The indecomposable objects of $\mathscr{F}_{(S, M)}$ are given by shifts of skyscraper sheaves on $\mathbb{A}^{1}$. The corresponding Lagrangians can be explicitly visualized in the annulus. The 
skyscraper sheaf of length $n$ with support at the origin in $\mathbb{A}^{1}$ corresponds to the curve which starts at the open end wraps $n$ times around the annulus and ends at the open end. A skyscraper sheaf of length $n$ with support at a point $\lambda \in \mathbb{A}^{1}$ with $\lambda \neq 0$, corresponds to a closed curve which wraps around the annulus $n$-times and is equipped with the flat $\mathbf{k}^{\star}$-principal bundle with monodromy $\lambda$. Shifting an indecomposable object amounts to changing the orientation of the corresponding object.

Dually, we can visualize the generator of the category $\mathscr{F}(S, M)$ corresponding to the vertex of the quiver $\mathcal{L}$. It corresponds to an arc which connects both boundary components of the annulus.

\subsubsection{The projective line}

Let $(S, M)$ be an annulus with one marked point on each boundary component. Figure 6 depicts two spanning Ribbon graphs and the real blow up.

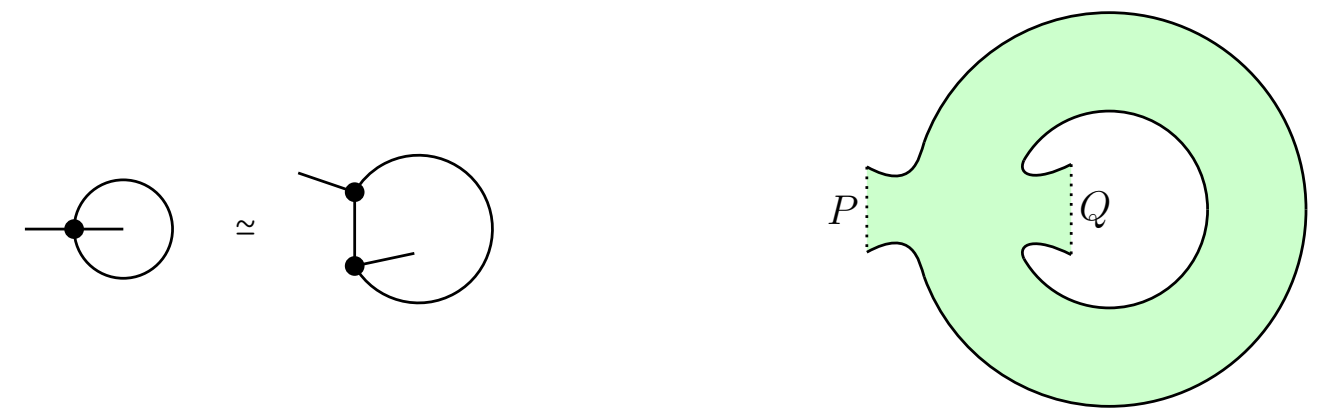

Figure 6: Spanning Ribbon graphs and real blowup

\section{Surface Postnikov system:}

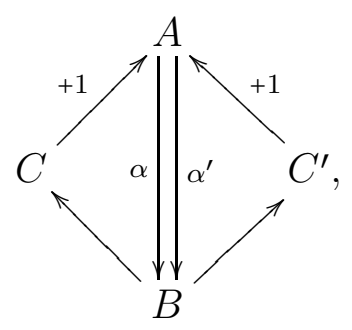

(Two distinguished triangles with two vertices in common.)

\section{Homotopy colimit presentation:}

$$
\mathscr{F}(S, M) \simeq\left(\mathcal{E}^{2} \coprod_{\mathcal{E}^{1}}^{\mathrm{h}} \mathcal{E}^{2}\right) \coprod_{\mathcal{E}^{1} \amalg \mathcal{E}^{1}}^{\mathrm{h}} \mathcal{E}^{1} \simeq\left(\mathcal{A}^{2} \coprod_{\mathcal{A}^{1}} \mathcal{A}^{2}\right) \coprod_{\mathcal{A}^{1} \amalg \mathcal{A}^{1}} \mathcal{A}^{1}=: \mathcal{K} .
$$

where we use a similar argument to simplify the homotopy colimit. The $\mathbb{Z} / 2 \mathbb{Z}$-graded $\mathbf{k}$-linear category $\mathcal{K}$ is freely generated by the Kronecker quiver with two vertices and two parallel arrows. It corresponds to the part $\left\{A \underset{\alpha^{\prime}}{\stackrel{\alpha}{\longrightarrow}} B\right\}$ in (4.2.2). 
Algebro-geometric picture: Using the classical equivalence $b$ of Beilinson [Bei78], we identify $\mathcal{F}^{(S, M)}$

$$
\begin{aligned}
& \mathscr{F}^{(S, M)} \simeq \operatorname{Perf}_{\mathcal{K}}^{(2)}=D^{(2)}(\mathcal{K}) \stackrel{b}{\longrightarrow} \operatorname{Perf}^{(2)}\left(\mathbb{P}_{\mathbf{k}}^{1}\right)=D^{(2)}\left(\mathbb{P}_{\mathbf{k}}^{1}\right), \\
& \left\{V_{A}^{\bullet} \stackrel{\alpha}{\stackrel{\alpha}{\longrightarrow}} V_{B}^{\bullet}\right\} \stackrel{b}{\longmapsto} \mathcal{P}^{\bullet}:=\operatorname{Cone}\left\{V_{A}^{\bullet} \otimes \mathcal{O}_{\mathbb{P}^{1}}(-1) \stackrel{\alpha t_{0}+\alpha^{\prime} t_{1}}{\longrightarrow} V_{B}^{\bullet} \otimes \mathcal{O}_{\mathbb{P}^{1}}\right\}
\end{aligned}
$$

with the $\mathbb{Z} / 2 \mathbb{Z}$-folding of the dg-category of perfect complexes on the projective line $\mathbb{P}_{\mathbf{k}}^{1}=$ $\operatorname{Proj} \mathbf{k}\left[t_{0}, t_{1}\right]$. Since this category is smooth and proper, and hence dualizable with respect to Morita duality, we obtain that

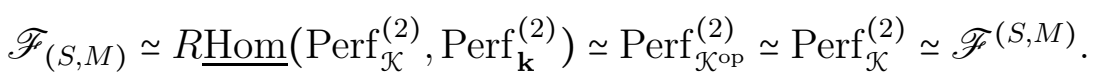

Again, we exhibit all indecomposable objects of $\operatorname{Perf}^{(2)}\left(\mathbb{P}_{\mathbf{k}}^{1}\right)$ explicitly as objects of $\mathscr{F}_{(S, M)}$, visualized as immersed Lagrangians in the annulus. The line bundle $\mathcal{O}(n), n \in \mathbb{Z}$, corresponds to an oriented arc starting at the open end $P$, wrapping $n$ times counterclockwise around the annulus and ending at the open end $Q$. The skyscraper sheaf of length $n$ supported at a finite nonzero point $\lambda$ in $\mathbb{P}_{k}^{1}$ corresponds to a closed oriented curve wrapping around the annulus $n$ times, equipped with the flat $\mathbf{k}^{*}$-principal bundle corresponding to $\lambda$. Skyscraper sheaves of length $n$ supported at 0 respectively $\infty$ correspond to oriented curves beginning and ending at $P$ respectively $Q$ wrapping around the annulus $n$ times.

Dually, we can visualize the generators of $\mathscr{F}(S, M)$ corresponding to the vertices of the Kronecker quiver.

\subsubsection{The nodal cubic curve}

Let $(S, M)$ be the torus with one marked point. We use the spanning Ribbon graph $\Gamma$ displayed in Figure 7.

\section{Surface Postnikov system:}

$$
A \underset{\alpha^{\prime}}{\stackrel{\alpha}{\longrightarrow}} B \underset{\beta^{\prime}}{\stackrel{\beta}{\longrightarrow}} C \underset{\gamma^{\prime}}{\stackrel{\gamma}{\longrightarrow}} \Sigma A,
$$

(Two distinguished triangles on the same 3 objects, having arrows in the same direction.) It differs from (4.2.2) in that $C \simeq \operatorname{Cone}(\alpha)$ is identified with $C^{\prime} \simeq \operatorname{Cone}\left(\alpha^{\prime}\right)$.

\section{Homotopy colimit presentation:}

$$
\mathscr{F}_{(S, M)} \simeq \operatorname{Perf}^{(2)}\left(\mathbb{P}_{\mathbf{k}}^{1}\right) \times{ }_{\operatorname{Perf}_{\mathbf{k}}^{(2)} \times \operatorname{Perf}_{\mathbf{k}}^{(2)}}^{\mathrm{h}} \operatorname{Perf}_{\mathbf{k}}^{(2)} .
$$

Algebro-geometric picture: In the Beilinson equivalence (4.2.3), the dg-vector spaces gives by the cones of $\alpha$ and $\alpha^{\prime}$ are the fibers of the perfect complex $\mathcal{P} \bullet$ at 0 and $\infty$. So an identification of these cones is a datum of descent to the nodal cubic curve $C=\mathbb{P}_{\mathbf{k}}^{1} /(0 \sim \infty)$. This can be extended to a Morita equivalence

$$
\mathscr{F}_{(S, M)} \simeq \operatorname{Perf}^{(2)}(C),
$$


see Sib12. Theorem 4.1.3 implies the existence of an action of $S L(2, \mathbb{Z})$ on $\operatorname{Perf}^{(2)}(C)$. Using the methods of [Sib12], the topological Fukaya categories associated to the Ribbon
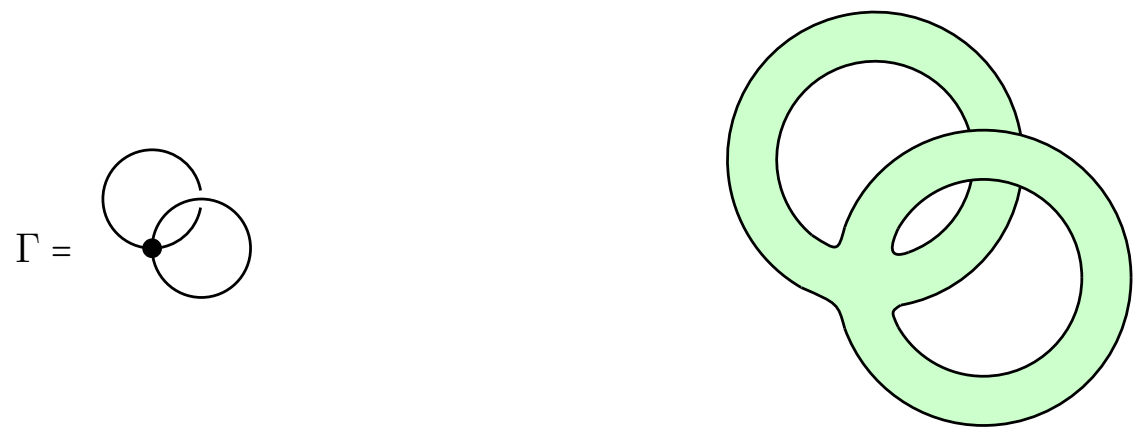

Figure 7: Ribbon graph $\Gamma$ and real blowup

graph

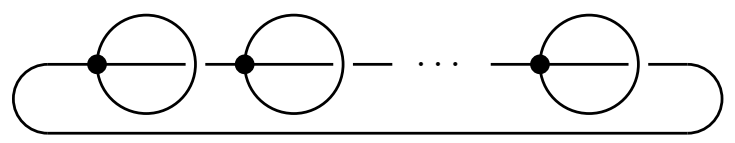

with $n$ vertices can be identified with the $\mathbb{Z} / 2 \mathbb{Z}$-folding of the category of perfect complexes on a cycle of $n$ projective lines which, by Theorem 4.1.3, comes equipped with an action of the mapping class group of the $n$-punctured torus. Note that, in [BK05, Sib12], actions of central extensions of these mapping class groups have been constructed on the $\mathbb{Z}$-graded variants of the above categories. When passing to 2-periodizations of the respective categories, these actions factor through the mapping class group actions which we construct.

\subsubsection{The union of two lines}

Let $(S, M)$ be a sphere with 3 marked points. Two (equivalent) spanning Ribbon graphs $\Gamma, \Gamma^{\prime}$ of $(S, M)$ are displayed in Figure 8 with the corresponding real blowup given by a disk with two open interior disks removed.
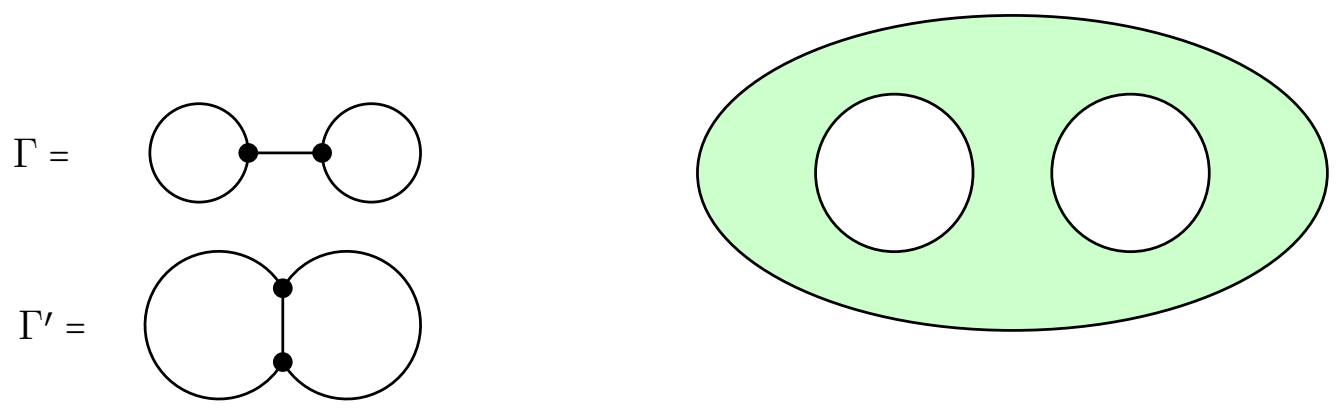

Figure 8: Ribbon graphs $\Gamma, \Gamma^{\prime}$ with corresponding real blowup 
Using the Ribbon graph $\Gamma^{\prime}$ we obtain the following.

\section{Surface Postnikov system:}

$$
A \underset{\alpha^{\prime}}{\stackrel{\alpha}{\rightleftarrows}} B \underset{\beta^{\prime}}{\stackrel{\beta}{\rightleftarrows}} C \underset{\gamma^{\prime}}{\stackrel{\gamma}{\rightleftarrows}} \Sigma A,
$$

(Two distinguished triangles on the same 3 objects, with arrows going in the opposite directions.)

\section{Homotopy colimit presentation:}

$$
\mathscr{F}(S, M) \simeq \mathcal{E}^{2} \coprod_{\mathcal{E}^{1} \amalg^{\mathcal{E}^{1}} \amalg^{\varepsilon^{1}}}^{\mathrm{h}} \mathcal{E}^{2} .
$$

Algebro-geometric picture: In (4.2.6), the endomorphisms

$$
y_{B}=\alpha \alpha^{\prime}, x_{B}=\beta^{\prime} \beta \in \operatorname{End}(B)
$$

satisfy $x_{B} y_{B}=y_{B} x_{B}=0$ and so make $B$ into a module (in $\mathcal{F}(S, M)$ ) over the algebra $R=\mathbf{k}[x, y] /(x y)$. Further, defining

$$
y_{A}=\alpha^{\prime} \alpha, x_{A}=(\Sigma \gamma)\left(\Sigma \gamma^{\prime}\right) \in \operatorname{End}(A), \quad y_{C}=\gamma^{\prime} \gamma, x_{C}=\beta \beta^{\prime} \in \operatorname{End}(C),
$$

we make $B$ and $C$ into $R$-modules as well, so that the arrows in (4.2.6) commute with the $R$-action. Using (4.2.6) as a tilting object and analyzing more carefully the morphisms of complexes, we construct a Morita equivalence $\mathcal{F}^{(S, M)} \simeq D_{\mathrm{fg}}^{(2)}(R)$ with the 2-periodification of the bounded derived category of finitely generated $R$-modules. On the level of objects, the equivalence takes $A \mapsto R /(x), B \mapsto R$, and $C \mapsto R /(y)$, and the surface Postnikov system (4.2.6) in $\mathscr{F}(S, M)$ corresponds to the system in $D(R)$ given by

$$
R /(x) \underset{\mathrm{pr}}{\stackrel{y}{\rightleftarrows}} R \underset{x}{\stackrel{\mathrm{pr}}{\rightleftarrows}} R /(y) \underset{\longleftrightarrow}{\rightleftarrows} R /(x)
$$

The mapping class group of a sphere with 3 marked points is the symmetric group $S_{3}$ (cf. [FM11]). Therefore, $S_{3}$ acts on $D_{\mathrm{fg}}^{(2)}(R)$ by equivalences of triangulated categories. The action on objects is given by

$$
\begin{array}{ll}
(12): & (R /(x), R, R /(y)) \mapsto(\Sigma R /(x), R /(y), R) \\
(23): & (R /(x), R, R /(y)) \mapsto(R, R /(x), \Sigma R /(y)) .
\end{array}
$$

For example, the cycle (123) induces a two-step rotation of the above distinguished triangles. Note that all three terms of the surface Postnikov system (4.2.8) have the same endomorphism ring

$$
\operatorname{Hom}_{R}(R, R) \cong \operatorname{Ext}_{R}^{\bullet}(R /(x), R /(x)) \cong \operatorname{Ext}_{R}^{\bullet}(R /(y), R /(y)) \cong R,
$$

in $D^{(2)}(R)$ due to the 2-periodic folding. 


\section{Application: Waldhausen S-construction}

In [HSS1], we showed that the Waldhausen S-construction of a stable $\infty$-category is a 2-Segal space. Generalizing results of [Lur11], it is shown in [Fao13] that the differential graded nerve $\mathrm{N}_{\mathrm{dg}}(\mathcal{A})$ of a perfect dg category $\mathcal{A}$ is a stable $\infty$-category.

In this section, we show that, given a 2-periodic perfect dg category $\mathcal{A}$, the Waldhausen S-construction of the stable $\infty$-category $\mathrm{N}_{\mathrm{dg}}(\mathcal{A})$ is weakly equivalent to the simplicial space $\operatorname{Map}(\varepsilon \bullet, \mathcal{A})$. An immediate consequence is the following result, predicted on a heuristic basis in [HSS1].

Theorem 5.0.1. The Waldhausen S-construction of a 2-periodic perfect dg category admits a canonical cyclic structure.

We recall the variant of the Waldhausen S-construction given in [HSS1]. For $n \geq 0$, let $\mathcal{J}^{n}$ be the nerve of the category Fun $([1],[n])$ corresponding to the poset formed by ordered pairs $(0 \leq i \leq j \leq n)$, with $(i, j) \leq(k, l)$ iff $i \leq k$ and $j \leq l$.

Definition 5.0.2. Let $\mathcal{C}$ be a stable $\infty$-category. We define

$$
\mathcal{S}_{n} \mathcal{C} \subset \operatorname{Fun}\left(J^{n}, \mathcal{C}\right)_{\text {Kan }}
$$

to be the simplicial subset given by those simplices whose vertices are $\mathfrak{d}^{n}$-diagrams $F$ satisfying the following conditions:

(1) For all $0 \leq i \leq n$, the object $F(i, i)$ is a zero object in $\mathcal{C}$.

(2) For any $0 \leq j \leq k \leq n$, the square

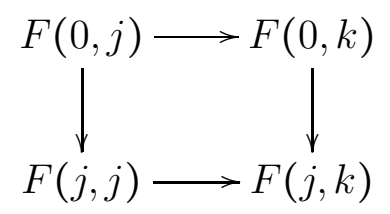

in $\mathcal{C}$ is coCartesian.

By construction, $\mathcal{S}_{n} \mathcal{C}$ is functorial in $[n]$ and defines a simplicial space $\mathcal{S} \mathcal{C}$, which we call the Waldhausen $S$-construction or Waldhausen space of $\mathcal{C}$.

To bridge between dg categories and $\infty$-categories, we use the Quillen adjunction

$$
\operatorname{dg}:\left(\text { Set }_{\Delta}, \text { Joyal }\right) \longleftrightarrow(\text { dgcat, } \mathrm{Qe}): \mathrm{N}_{\mathrm{dg}}
$$

as introduced in [Lur11. Here, dgcat is equipped with the quasi-equivalence model structure of Tabuada and $\mathcal{S e t}_{\Delta}$ is equipped with the quasi-category model structure of Joyal. Further, we have the Quillen adjunction

$$
P:(\text { dgcat }, \mathrm{Qe}) \longleftrightarrow\left(\operatorname{dgcat}^{(2)}, \mathrm{Qe}\right): F
$$


from (1.2.2), where $P$ is given by folding the mapping complexes 2-periodically and $F$ is the functor which forgets 2-periodicity. In what follows, we leave the application of $F$ implicit.

There is a canonical dg functor $U^{n}: \operatorname{dg}\left(g^{n}\right) \rightarrow \mathcal{E}^{n}$ extending the assignment

$$
(i, j) \mapsto E_{i j}
$$

which, under (5.0.3) , is adjoint to the functor $\mathrm{g}^{n} \rightarrow \mathrm{N}_{\mathrm{dg}}\left(\mathcal{E}^{n}\right)$ which is nontrivial only on the 1-skeleton of $g^{n}$ and sends the edge $(i, j) \leq(k, l)$ of $g^{n}$ to the edge of $\mathrm{N}_{\mathrm{dg}}\left(\mathcal{E}^{n}\right)$ given by the closed morphism of degree 0

$$
\left(\begin{array}{cc}
z^{k-i} & 0 \\
0 & z^{l-j}
\end{array}\right): E_{i j} \longrightarrow E_{k l}
$$

By [Hir03, 17.4.15], the above Quillen adjunctions induce weak equivalences of mapping spaces

$$
\operatorname{Map}_{\left(\operatorname{dgcat}^{(2)}, \mathrm{Qe}\right)}\left(P\left(\operatorname{dg}\left(g^{n}\right)\right), \mathcal{A}\right) \simeq \operatorname{Map}_{(\operatorname{dgcat}, \mathrm{Qe})}\left(\operatorname{dg}\left(\mathcal{g}^{n}\right), \mathcal{A}\right) \simeq \operatorname{Map}_{\left(\operatorname{Set}_{\Delta}, \mathrm{Joyal}\right)}\left(\mathcal{J}^{n}, \mathrm{~N}_{\mathrm{dg}}(\mathcal{A})\right) .
$$

Further, we have the formula

$$
\operatorname{Map}_{\left(\operatorname{Set}_{\Delta}, \text { Joyal }\right)}\left(\mathcal{J}^{n}, \mathrm{~N}_{\mathrm{dg}}(\mathcal{A})\right) \simeq \operatorname{Fun}\left(\mathcal{J}^{n}, \mathrm{~N}_{\mathrm{dg}}(\mathcal{A})\right)_{\mathrm{Kan}}
$$

for the mapping spaces with respect to the Joyal model structure on $\mathcal{S e t}_{\Delta}$. Hence, pullback along the functor $U^{n}$ gives a natural map of simplicial sets

$$
\left(U^{n}\right)^{*}: \operatorname{Map}^{(2)}\left(\mathcal{E}^{n}, \mathcal{A}\right) \longrightarrow \operatorname{Fun}\left(\mathcal{J}^{n}, \mathrm{~N}_{\mathrm{dg}}(\mathcal{A})\right)_{\mathrm{Kan}} \cdot
$$

The following proposition shows that the functor $U^{n}$ is the universal Waldhausen $n$ simplex.

Proposition 5.0.5. For every $n \geq 0$, the pullback map $\left(U^{n}\right)^{*}$ factors into

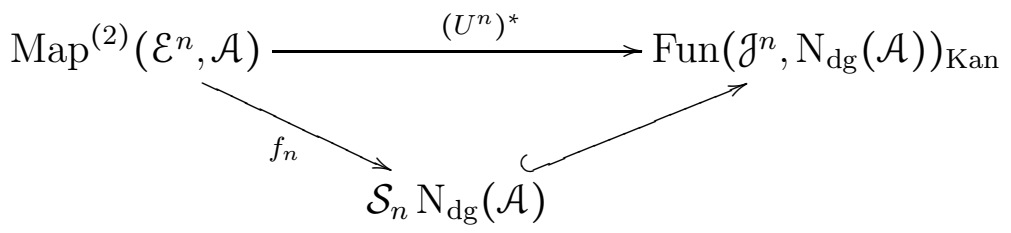

where the map $f_{n}$ is a weak equivalence. In other words, the functor $\left(U^{n}\right)^{*}$ is a weak equivalence onto the union of those connected components $\operatorname{Fun}\left(\mathcal{J}^{n}, \mathrm{~N}_{\mathrm{dg}}(\mathcal{A})\right)_{\text {Kan }}$ which satisfy conditions (1) and (2) of Definition 5.0.2. Further, the resulting weak equivalences $\left\{f_{n}\right\}$ assemble to provide a weak equivalence of simplicial spaces

$$
\operatorname{Map}^{(2)}\left(\mathcal{E}^{\bullet}, \mathcal{A}\right) \stackrel{\simeq}{\longrightarrow} \mathcal{S}_{\bullet} \mathrm{N}_{\mathrm{dg}}(\mathcal{A}) \text {. }
$$


Proof. We utilize the $\infty$-categorical theory of Kan extensions as developed in [Lur09a]. Consider the functor $i: \Delta^{n-1} \rightarrow g^{n}$ given by the apparent extension of the association

$$
k \mapsto[0, k]
$$

on objects. Consider the commutative diagram of simplicial sets

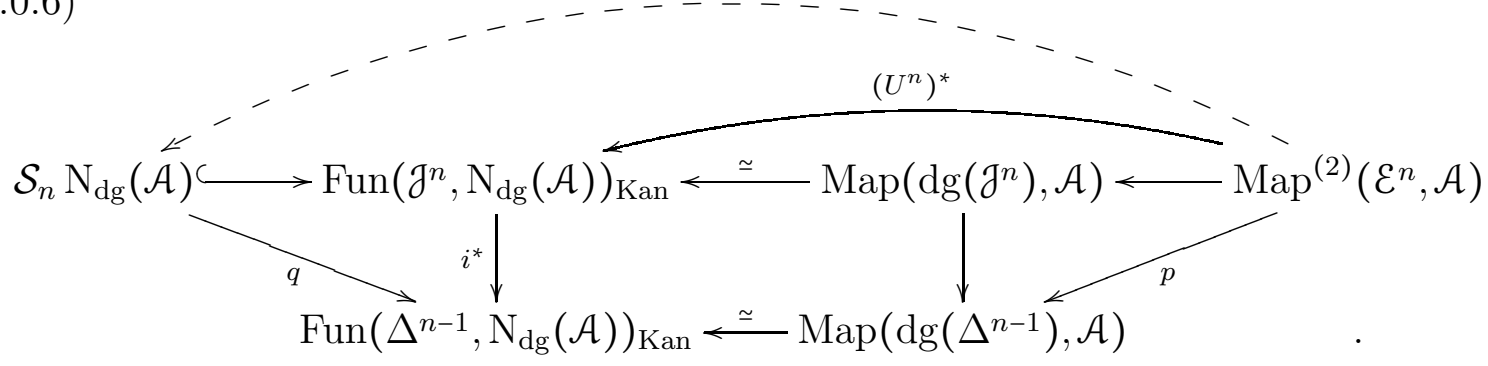

The map $q$ is a weak equivalence by Proposition 7.3.6(1) in [HSS1]. The map $p$ is a weak equivalence, since it is given by pulling back along the composite

$$
\operatorname{dg}\left(\Delta^{n-1}\right) \stackrel{p_{1}}{\longrightarrow} \mathcal{A}^{n} \stackrel{r_{n}^{\triangleleft}}{\longrightarrow} \mathcal{E}^{n}
$$

where $p_{1}$ is a quasi-equivalence and $r_{n}^{\triangleleft}$ is the Morita equivalence from Proposition 2.4.6. We claim that the map $\left(U^{n}\right)^{*}$ factors as indicated by the dashed arrow in (5.0.6). Assuming the existence of this factorization, we obtain a commutative diagram

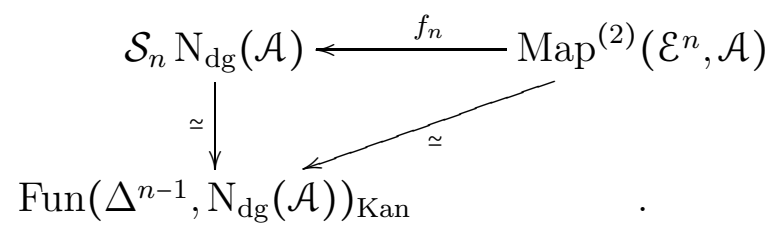

which shows that $f_{n}$ is a weak equivalence.

To show the claimed factorization, note that, since $\mathcal{S}_{n} \mathrm{~N}_{\mathrm{dg}}(\mathcal{A})$ is a union of connected components of $\operatorname{Fun}\left(\mathcal{J}^{n}, \mathrm{~N}_{\mathrm{dg}}(\mathcal{A})\right)_{\mathrm{Kan}}$, it suffices to show that the factorization exists after passing to $\pi_{0}$, i.e., morphism sets in the respective homotopy categories. Choosing a cofibrant replacement $\widetilde{\mathcal{E}^{n}} \rightarrow \mathcal{E}^{n}$, we express pullback by $U^{n}$ as the composite

$$
\left[\mathcal{E}^{n}, \mathcal{A}\right] \rightarrow\left[\widetilde{\mathcal{E}^{n}}, \mathcal{A}\right] \rightarrow\left[\operatorname{dg}\left(\mathcal{J}^{n}\right), \mathcal{A}\right] \cong\left[\mathcal{J}^{n}, \mathrm{~N}_{\mathrm{dg}}(\mathcal{A})\right]
$$

which allows us to represent every element of $\left[\widetilde{\varepsilon^{n}}, \mathcal{A}\right]$ by a $\operatorname{dg}$ functor $\widetilde{\varepsilon^{n}} \rightarrow \mathcal{A}$. Using the method of [Lur11, 1.3.2] one verifies that the squares

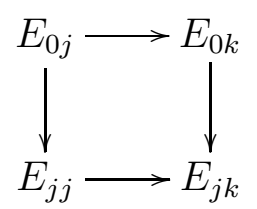


in $\mathcal{E}^{n}$, become homotopy pushout squares in the model category $\left(\mathcal{E}^{n}\right)^{\text {op }}$-mod. The claim now follows from the general comparison result [Lur09a, 4.2.4.1] between homotopy limits in simplicial categories and $\infty$-categorical limits, again using the methods of [Lur11, 1.3.2]: the homotopy pushout square (5.0.9) becomes an $\infty$-categorical pushout square of $\mathrm{N}_{\mathrm{dg}}\left(\operatorname{Perf}\left(\mathcal{\varepsilon}^{n}\right)\right)$. This implies that its lift in $\mathrm{N}_{\mathrm{dg}}\left(\operatorname{Perf}\left(\widetilde{\varepsilon^{n}}\right)\right)$ is a pushout square which maps to a pushout square in $\mathcal{A}$ (since dg functors between perfect dg categories preserve finite homotopy colimits). This implies that the image of the composite (5.0.8) satisfies condition (2) of Definition 5.0.2, It also satisfies condition (1) by applying the same argument to the (homotopy) zero objects $E_{i i}$ of $\left(\varepsilon^{n}\right)^{\text {op }}$-mod.

\section{References}

[An12] Y. André. Uniform sheaves and differential equations. ArXiv e-prints, April 2012, 1207.3380 .

[Bei78] A. A. Beilinson. Coherent sheaves on $\mathbb{P}^{n}$ and problems of linear algebra. Funkt. Anal. Appl. 12(3):214-216, 1978.

[BK91] A. I. Bondal, M. M. Kapranov. Enhanced triangulated categories. Math. USSR. Sbornik, 70(1): 93-107, 1991.

[BK05] I. Burban, B. Kreussler. Fourier-Mukai transforms and semi-stable sheaves on nodal Weierstraßcubics. J. Reine Angew. Math. 584: 45-82, 2005.

[BLL04] A. I. Bondal, M. Larsen, V. A. Lunts. Grothendieck ring of pretriangulated categories. Int. Math. Res. Not. 29: 461-495, 2004.

[Con94] A. Connes. Noncommutative Geometry. Academic Press, San Diego, New York, London, 1994.

[Dr04] V. Drinfeld. On the notion of geometric realization. Mosc. Math. J. 4(3): 619-626 (2004).

[DHKS04] W. G. Dwyer, P. S. Hirschhorn, D. M. Kan, and J. H. Smith. Homotopy limit functors on model categories and homotopical categories, volume 113 of Mathematical Surveys and Monographs. American Mathematical Society, Providence, RI, 2004.

[Hir03] P. S. Hirschhorn. Model categories and their localizations, volume 99 of Mathematical Surveys and Monographs. American Mathematical Society, Providence, RI, 2003.

[DHK85] W. G. Dwyer, M. J. Hopkins, D. M. Kan. The homotopy theory of cyclic sets. Trans. AMS, 291:281-289, 1985. 
[Dyc11] T. Dyckerhoff. Compact generators in categories of matrix factorizations. Duke Math. J. 159:223-274, 2011.

[HSS1] T. Dyckerhoff, M. Kapranov. Higher Segal spaces I. ArXiv e-prints, December $2012,1212.3563$.

[HSS2] T. Dyckerhoff, M. Kapranov. Higher Segal spaces II. In preparation.

[Eis80] D. Eisenbud. Homological algebra on a complete intersection, with an application to group representations. Trans. Amer. Math. Soc. 260: 35-64, 1980.

[Fao13] G. Faonte. Simplicial Nerve of an $A_{\infty}$-category, in preparation.

[GM03] S. I. Gelfand, Y. I. Manin. Methods of Homological Algebra. Springer-Verlag, Berlin, 2003.

[FG06] V. Fock and A. Goncharov. Moduli spaces of local systems and higher Teichmüller theory. Publ. Math. Inst. Hautes Études Sci., (103):1-211, 2006.

[FST08] S. Fomin, M. Shapiro, and D. Thurston. Cluster algebras and triangulated surfaces. I. Cluster complexes. Acta Math., 201(1):83-146, 2008.

[Gil81] H. Gillet. Riemann-Roch theorems for higher algebraic K-theory. Advances in Mathematics, 40(3):203-289, 1981.

[Hap87] D. Happel. On the derived category of finite-dimensional algebra. Comm. Math. Helv. 62:339-389, 1987.

[Har86] J. L. Harer. The virtual cohomological dimension of the mapping class group of an orientable surface. Invent. Math., 84(1):157-176, 1986.

[Hat91] A. Hatcher. On triangulations of surfaces. Topology Appl., 40(2):189-194, 1991.

[Hat02] A. Hatcher. Algebraic topology. Cambridge University Press, Cambridge, 2002.

[Hov99] M. Hovey. Model categories, volume 63 of Mathematical Surveys and Monographs. American Mathematical Society, Providence, RI, 1999.

[Hun16] E. V. Huntington. A set of independent postulates for cyclic order. Proc. Nat. Acad. of Sci. USA, 11(2):630-631, 1916.

[Igu02] K. Igusa. Higher Franz-Reidemeister torsion, volume 31 of AMS/IP Studies in Advanced Mathematics. American Mathematical Society, Providence, RI, 2002.

[KST07] H. Kajiura, K. Saito, A. Takahashi. Matrix factorizations and representations of quivers II: Type ADE case. Advances in Math. 211(1): 327-362, 2007. 
[Kel94] B. Keller. Deriving dg-categories. Ann. Sci. Éc. Norm. Sup. 27:63-102, 1994.

[Kel99] B. Keller. On the cyclic homology of exact categories. J. Pure Appl. Algebra, 136(1):1-56, 1999.

[Kon09] M. Kontsevich. Singular Lagrangian branes. Talk at Miami Conference on Mirror Symmetry, Jan. 2009.

[Kon09'] M. Kontsevich. Symplectic geometry of homological algebra. Preprint, June 2009, available at http://www.ihes.fr/ maxim/publicationsfrancais.html

[Lur06] J. Lurie. Stable $\infty$-categories. ArXiv e-prints, August 2006, math/0608228.

[Lur09a] J. Lurie. Higher topos theory, volume 170 of Annals of Mathematics Studies. Princeton University Press, Princeton, NJ, 2009.

[Lur11] J. Lurie. Higher Algebra. preprint, May 2011, available at the author's homepage.

[FM11] B. Farb, D. Margalit. A primer on mapping class groups. Princeton Univ. Press, 2011.

[Mar06] M. Markl. Transferring $A_{\infty}$ (strongly homotopy associative) structures. Rend. Circ. Math. Palermo (2) Suppl. No. 79: 139-151, 2006.

[Nad11] D. Nadler. Fukaya categories as categorical Morse homology. ArXiv e-prints, September 2011, 1109.4848.

[Nad13] D. Nadler. Cyclic symmetries of $A_{n}$-quiver representations. ArXiv e-prints, June 2013, 1306.0070.

[Pen10] R. C. Penner. Decorated Teichmüller Theory. European Math. Soc. Publ. Zürich, 2010.

[Rez01] C. Rezk. A model for the homotopy theory of homotopy theory. Trans. Amer. Math. Soc., 353(3):973-1007 (electronic), 2001.

[Sei08] P. Seidel. Fukaya Categories and Picard-Lefschetz Theory. European Math. Soc. Publ. Zürich, 2008.

[Sib12] N. Sibilla. HMS for punctured tori and categorical mapping class group actions. In String-Math 2011, volume 85 of Proc. Sympos. Pure Math., pages 467-476. Amer. Math. Soc., Providence, RI, 2012.

[STZ11] N. Sibilla, D. Treumann, E. Zaslow. Ribbon graphs and mirror symmetry I. ArXiv e-prints, March 2011, 1103.2462. 
[Sta63] J. D. Stasheff. Homotopy associativity of $H$-spaces. I, II. Trans. Amer. Math. Soc. 108 (1963), 275-292; ibid., 108:293-312, 1963.

[Tab07] G. N. Tabuada. Théorie homotopique des DG-catégories. Thesis, Université Paris-7, 2007. ArXiv e-prints, October 2007, 0710.4303.

[Tak05] A. Takahashi. Matrix factorizations and representations of quivers I. ArXiv e-prints, June 2005, math.AG/0506347.

[Toё07] B. Toën. The homotopy theory of dg-categories and derived Morita theory. Invent. Math. 167: 615-667, 2007.

[TV07] B. Toen, M. Vaquié. Moduli of objects in dg-categories. Ann. Sci. Éc. Norm. Sup. 40:387-444, 2007.

[Wh70] G. W. Whitehead. Recent Advances in Homotopy Theory. (CBMS Regional Conference Series in Mathematics, No 5.) American Mathematical Society, Prividence, 1970. 